\title{
OSCILLATORY BEHAVIOR OF LARGE EIGENVALUES IN QUANTUM RABI MODELS
}

\author{
ANNE BOUTET DE MONVEL ${ }^{1}$ AND LECH ZIELINSKI ${ }^{2}$
}

\begin{abstract}
We investigate the large $n$ asymptotics of the $n$-th eigenvalue for a class of unbounded self-adjoint operators defined by infinite Jacobi matrices with discrete spectrum. In the case of the quantum Rabi model we obtain the first three terms of the asymptotics which determine the parameters of the model. This paper is based on our previous paper [5] that it completes and improves.
\end{abstract}

\section{INTRODUCTION}

1.1. The quantum Rabi model. This paper is motivated by the quantum Rabi model 8 describing the simplest interaction between radiation and matter (see [17]). It is also called the Jaynes-Cummings model without the rotating-wave approximation. The Hamiltonian of this model is a self-adjoint operator $H_{\text {Rabi }}$ with discrete spectrum defined in Appendix. We refer to 21] for a list of recent works on this model.

A natural question is whether the spectrum of $H_{\text {Rabi }}$ determines the values of all the parameters involved in its definition. These parameters are listed in the Appendix and denoted by $\omega, E$, and $g$. The answer to this question is positive. In [6] we explain how to recover the values of all the parameters from the spectrum of $H_{\text {Rabi }}$. The method described in $[6]$ is based on the three term asymptotics for large eigenvalues of $H_{\text {Rabi }}$. This asymptotic formula can be easily deduced from the formula (1.4a) of this paper using the well known fact (see [19,20,22]) that $H_{\text {Rabi }}$ can be written as the direct sum of two Jacobi matrices (see Appendix) to which Theorem 1.1 applies.

Further on $J$ denotes an infinite real Jacobi matrix

$$
J=\left(\begin{array}{ccccc}
d(1) & a(1) & 0 & 0 & \ldots \\
a(1) & d(2) & a(2) & 0 & \ldots \\
0 & a(2) & d(3) & a(3) & \ldots \\
0 & 0 & a(3) & d(4) & \ldots \\
\vdots & \vdots & \vdots & \vdots & \ddots
\end{array}\right) .
$$

To treat the Jacobi matrices representing the quantum Rabi model we have to consider entries $\{d(k)\}_{k=1}^{\infty}$ and $\{a(k)\}_{k=1}^{\infty}$ of the form

$$
\left\{\begin{array}{l}
d(k)=k+(-1)^{k} \rho \\
a(k)=a_{1} k^{1 / 2}
\end{array}\right.
$$

where $\rho \in \mathbb{R}$ and $a_{1}>0$ are some constants.

The Jacobi matrix (1.1) defines the self-adjoint operator $J$ that acts on $l^{2}\left(\mathbb{N}^{*}\right)$ by

$$
(J x)(k)=d(k) x(k)+a(k) x(k+1)+a(k-1) x(k-1)
$$

2010 Mathematics Subject Classification. Primary 47B36; Secondary 81T10, 81Q10, 47A75, 47A55.

Key words and phrases. Jacobi matrices, quantum Rabi model, Jaynes-Cummings model, eigenvalue estimates. 
where, by convention, $x(0)=0$ and $a(0)=0$. We denote by $\mathbb{N}^{*}$ the set of positive integers and by $l^{2}\left(\mathbb{N}^{*}\right)$ the Hilbert space of square summable complex sequences $x=(x(k))_{k=1}^{\infty}$ equipped with the scalar product $\langle x, y\rangle=\sum_{k=1}^{\infty} \overline{x(k)} y(k)$. The operator $J$ is defined on $\mathcal{D}:=\left\{x \in l^{2}\left(\mathbb{N}^{*}\right)\right.$ : $\left.\sum_{k=1}^{\infty} d(k)^{2}|x(k)|^{2}<\infty\right\}$.

Under our assumptions, in particular under (1.2), the self-adjoint operator $J$ is bounded from below with compact resolvent. Its spectrum is therefore discrete and one can find an orthogonal basis $\left\{w_{n}\right\}_{n=1}^{\infty}$ such that $J w_{n}=\lambda_{n}(J) w_{n}$ where $\left\{\lambda_{n}(J)\right\}_{n=1}^{\infty}$ is the non-decreasing sequence of its eigenvalues:

$$
\lambda_{1}(J) \leq \cdots \leq \lambda_{n}(J) \leq \lambda_{n+1}(J) \leq \cdots
$$

The aim of this paper is to describe the asymptotic behavior of $\lambda_{n}(J)$ when $n \rightarrow \infty$.

Theorem 1.1 (Quantum Rabi model). Let $J$ be defined by (1.3) with $\{a(k)\}_{k=1}^{\infty}$ and $\{d(k)\}_{k=1}^{\infty}$ given by (1.2). Let $\lambda_{n}(J)$ denote the $n$-th eigenvalue of $J$. Then, for any $\varepsilon>0$ we have the large $n$ asymptotic formula

$$
\lambda_{n}(J)=n-a_{1}^{2}+\mathfrak{r}(n)+\mathrm{O}\left(n^{-1 / 2+\varepsilon}\right),
$$

where

$$
\mathfrak{r}(n)=(-1)^{n} \rho \frac{\cos \left(4 a_{1} \sqrt{n}-\frac{\pi}{4}\right)}{\sqrt{2 \pi a_{1}}} n^{-1 / 4} .
$$

1.2. Comments. In this section $J$ denotes the Jacobi operator defined by (1.3)-(1.2).

1.2.1. The three-term asymptotics (1.4) improves the two-term asymptotics proved by Yanovich 22] (see also an earlier version in [20]):

$$
\lambda_{n}(J)=n-a_{1}^{2}+\mathrm{O}\left(n^{-1 / 16}\right) .
$$

1.2.2. The large $n$ behavior of $\lambda_{n}(J)$ was already investigated by Schmutz [16]. Let $J_{0}$ denote the operator defined by (1.3)-(1.2) when $\rho=0$, i.e.,

$$
\left(J_{0} x\right)(k)=k x(k)+a_{1} \sqrt{k} x(k+1)+a_{1} \sqrt{k-1} x(k-1) .
$$

As noticed by Schmutz, $J_{0}$ can be diagonalized in the canonical basis by means of the Bogoliubov transformation:

$$
L_{0}:=\mathrm{e}^{\mathrm{i} B} J_{0} \mathrm{e}^{-\mathrm{i} B}=\operatorname{diag}\left(k-a_{1}^{2}\right)_{k=1}^{\infty}
$$

defined by $B=a_{1}\left(\hat{a}-\hat{a}^{\dagger}\right)$, where $\hat{a}$ and $\hat{a}^{\dagger}$ are the annihilation and creation operators, respectively (see Appendix A). Since $J=J_{0}+V$ with $V=\operatorname{diag}\left((-1)^{k} \rho\right)$, its eigenvalues are the same as those of

$$
L:=\mathrm{e}^{\mathrm{i} B} J \mathrm{e}^{-\mathrm{i} B}=L_{0}+\tilde{V},
$$

where $\tilde{V}:=\mathrm{e}^{\mathrm{i} B} V \mathrm{e}^{-\mathrm{i} B}$. Then, decay properties of the entries of $\tilde{V}$ should allow to expect the large $n$ behavior

$$
\lambda_{n}(J)=\lambda_{n}(L) \approx \lambda_{n}\left(L_{0}\right)=n-a_{1}^{2} .
$$

1.2.3. The asymptotic behavior of the matrix elements $\tilde{V}(j, k)$ expressed by means of Laguerre polynomials was considered by I. D. Feranchuk et al [9] who proposed to apply the 0th order perturbation theory (see the book [10]) and suggested the following improvement of (1.7):

$$
\lambda_{n}(J)=\lambda_{n}(L) \approx \lambda_{n}\left(L_{0}\right)+\tilde{V}(n, n) .
$$

The approximation (1.8) was discovered independently by Irish [12]. Following Irish, (1.8) is called the Generalized Rotating-Wave Approximation (GRWA) in the physical literature. We observe that one can prove that

$$
\tilde{V}(n, n)=\mathfrak{r}(n)+\mathrm{O}\left(n^{-1 / 2+\varepsilon}\right),
$$

where $\mathfrak{r}(n)$ is given by (1.4b). 
1.2.4. The first step of our analysis uses an analog of the Bogoliubov transformation. In order to simplify the remainder estimates with respect to the large parameter $n$ we work with Jacobi operators indexed by $n$ and write

$$
\mathrm{e}^{\mathrm{i} B_{n}} J_{n} \mathrm{e}^{-\mathrm{i} B_{n}}=L_{0, n}+\tilde{V}_{n}+R_{n}
$$

where $L_{0, n}$ is diagonal, $\left\|R_{n}\right\|=\mathrm{O}\left(n^{-1 / 2}\right)$, and

$$
\tilde{V}_{n}(n, n)=\mathfrak{r}(n)+\mathrm{O}\left(n^{-1 / 2+\varepsilon}\right) .
$$

The definitions of $J_{n}, L_{0, n}$, and $\tilde{V}_{n}$ are given in Section 2.2. Propositions 2.1 and 2.3 ensure the fact that the asymptotic formula for $J$ can be reduced to an analogous formula for $J_{n}$ and $L_{n}=L_{0, n}+\tilde{V}_{n}$.

1.2.5. The asymptotic behavior of $\lambda_{n}\left(J_{n}\right)$ is deduced from the trace estimate described in Section 2.3 by means of a Tauberian type result [5, Proposition 11.1] slightly adapted in Proposition 2.5. We begin the proof of the trace estimate in Section 4 by reducing the problem to large $n$ estimates of a Dyson expansion similarly as in [5. We notice that Section 4 is the only part of this paper where we rewrite proofs from [5] in a slightly more general form. We complete the proof by an analysis of the Dyson expansion in Sections 5 [ 8 and to perform this analysis we need to use a certain number of auxiliary results from [5, Section 10]. In order to avoid unnecessary overlaps we refer to [5] for the proofs of these auxiliary results.

1.2.6. In Section 1.3 we describe a class of more general type of operators for which we can obtain an analogous large $n$ asymptotic formula. Following [1] we replace the sequence $\rho(-1)^{k}$ by a general sequence of period $N$ and we give the corresponding asymptotic formula in Theorem 1.2 Since in practice the proofs of Theorem 1.1 and Theorem 1.2 require the same arguments, we chose to present the proof in the more general framework, i.e., for the class of operators described in Section 1.3. For readers interested only in the result of Theorem 1.1 we indicate that the only simplification with respect to Theorem 1.2 consists in the fact that the proof of (1.9) is simpler in the case of period $N=2$. Indeed, an additional symmetry of this case allows us to express an approximation of $\tilde{V}_{n}(k, k)$ by oscillating integrals with very simple phase functions (see Section 3.2) and to obtain (1.9) immediately from the stationary phase formula. Thus the proof of Theorem 1.1 ends in Section 8.1. In the case of Theorem 1.2 the proof of (1.9) involves more complicated phase functions and is given in Section 8.2 .

1.2.7. Our approach works the same way in the proofs of Theorems 1.1 and 1.2 Therefore, it does not distinguish whether or not the corresponding model is integrable in the sense of Braak [7. For this reason, it makes no contribution to the Braak conjecture.

1.3. Quantum Rabi type models. We consider the following assumptions on the entries of $J$ :

Assumption (H1). There exist constants $0<\gamma \leq \frac{1}{2}, C, C^{\prime}, C^{\prime \prime}$, and $c>0$ such that

$$
\begin{aligned}
& c k^{\gamma} \leq a(k) \leq C k^{\gamma}, \\
& |\delta a(k)| \leq C^{\prime} k^{\gamma-1}, \\
& \left|\delta^{2} a(k)\right| \leq C^{\prime \prime} k^{\gamma-2}
\end{aligned}
$$

for any $k \in \mathbb{N}^{*}$. Here, $\delta a(k):=a(k+1)-a(k)$ and $\delta^{2} a(k):=a(k+2)-2 a(k+1)+a(k)$.

Remark. (H1) is satisfied if $a(k)$ has the large $k$ behavior

$$
a(k)=a_{1} k^{\gamma}+a_{1}^{\prime} k^{\gamma-1}+\mathrm{O}\left(k^{\gamma-2}\right) .
$$


Assumption (H2). The diagonal entries of $J$ are of the form

$$
d(k)=k+v(k)
$$

where $v: \mathbb{N}^{*} \rightarrow \mathbb{R}$ is real-valued and periodic of period $N$, i.e.:

$$
v(k+N)=v(k) \text { for any } k \in \mathbb{N}^{*} .
$$

Moreover, we assume

$$
\rho_{N}< \begin{cases}\frac{1}{2} & \text { if } N=2, \\ \frac{1}{\pi \sqrt{N}} & \text { if } N \geq 3\end{cases}
$$

where

$$
\rho_{N}=\rho_{N}(v):=\max _{1 \leq k \leq N}|v(k)-\langle v\rangle| .
$$

Here $\langle v\rangle:=\frac{1}{N} \sum_{1 \leq k \leq N} v(k)$ denotes the "mean value" of $v$.

To compare with the hypotheses of Theorem 1.1 we reformulate these as follows:

Assumption (H0). The diagonal and off-diagonal entries of $J$ are of the form

$$
\begin{cases}d(k)=k+v(k), & \text { with } v(k)=(-1)^{k} \rho, \\ a(k)=a_{1} k^{\gamma}, & \text { with } \gamma=\frac{1}{2},\end{cases}
$$

where $\rho$ is a real constant. In particular, $v$ is periodic of period $N=2,\langle v\rangle=0$, and $\rho_{N}=|\rho|$. Remark. (H0) is a special case of $(\mathrm{H} 1)$ and $(\mathrm{H} 2)$, except that there is no restriction on $\rho_{N}=|\rho|$.

Let $v$ be as in (H2). By $N$-periodicity we can expand it as follows:

$$
v(k)=\alpha_{0}+\sum_{m=1}^{\lfloor N / 2\rfloor} \alpha_{m} \cos \frac{2 m \pi k}{N}+\sum_{m=1}^{\lfloor(N-1) / 2\rfloor} \tilde{\alpha}_{m} \sin \frac{2 m \pi k}{N},
$$

where

- $\lfloor s\rfloor:=\max \{k \in \mathbb{Z}: k \leq s\}$ is the integer part of $s$,

- all coefficients $\alpha_{0}=\langle v\rangle, \alpha_{m}$, and $\tilde{\alpha}_{m}$ are real constants.

Theorem 1.2 (Quantum Rabi type model). Let $J$ be defined by (1.3) with $\{a(k)\}_{k=1}^{\infty}$ and $\{d(k)\}_{k=1}^{\infty}$ satisfying assumptions (H1) and (H2), respectively. Then, for any $\varepsilon>0$ we have the large $n$ asymptotic formula:

$$
\lambda_{n}(J)=n+a(n-1)^{2}-a(n)^{2}+\alpha_{0}+\mathfrak{r}(n)+\mathrm{O}\left(n^{-\gamma+\varepsilon}\right) .
$$

where $\alpha_{0}=\langle v\rangle$ and

$$
\mathfrak{r}(n)=\sum_{m=1}^{\lfloor N / 2\rfloor} \alpha_{m} \mathfrak{r}_{m}(n)+\sum_{m=1}^{\lfloor(N-1) / 2\rfloor} \tilde{\alpha}_{m} \tilde{\mathfrak{r}}_{m}(n),
$$

with $\alpha_{m}, \tilde{\alpha}_{m}$ as in (1.12) and $\mathfrak{r}_{m}(n), \tilde{\mathfrak{r}}_{m}(n)$ defined by

$$
\begin{aligned}
& \mathfrak{r}_{m}(n):=\frac{\cos \left(4 a(n) \sin \frac{m \pi}{N}-\frac{\pi}{4}\right)}{\sqrt{2 \pi a(n) \sin \frac{m \pi}{N}}} \cos \left(\frac{2 m \pi n}{N}+2 a(n) \delta a(n) \sin \frac{2 m \pi}{N}\right), \\
& \tilde{\mathfrak{r}}_{m}(n):=\frac{\cos \left(4 a(n) \sin \frac{m \pi}{N}-\frac{\pi}{4}\right)}{\sqrt{2 \pi a(n) \sin \frac{m \pi}{N}}} \sin \left(\frac{2 m \pi n}{N}+2 a(n) \delta a(n) \sin \frac{2 m \pi}{N}\right) .
\end{aligned}
$$


Remark. For $N=2$, the expression of $\mathfrak{r}(n)$ reduces to

$$
\mathfrak{r}(n)=\rho \mathfrak{r}_{1}(n)=(-1)^{n} \rho \frac{\cos \left(4 a(n)-\frac{\pi}{4}\right)}{\sqrt{2 \pi a(n)}} .
$$

Moreover, in the case of the quantum Rabi model, $a(n)=a_{1} \sqrt{n}$, hence $a(n-1)^{2}-a(n)^{2}=-a_{1}^{2}$. Then, (1.13) becomes the asymptotic formula (1.4) in Theorem 1.1.

Assumption. Further on, we make the assumption

$$
\langle v\rangle=\alpha_{0}=0 .
$$

Indeed, since $\lambda_{n}(J)=\alpha_{0}+\lambda_{n}\left(J-\alpha_{0} I\right)$ it suffices to prove Theorem 1.2 for $J-\alpha_{0} I$.

1.4. Plan of the paper. As in [5] the main ingredient of our approach is a trace estimate (Proposition 2.4). In Section 2 we show the implication

$$
\left.\begin{array}{l}
\text { Propositions } 2.1 \& \text { 2.3 } \\
\text { Propositions } 2.4 \& 2.5 \\
\text { Lemmas } 2.2 \& 2.7
\end{array}\right\} \Longrightarrow \text { Theorems } 1.1 \& 1.2 .
$$

Section 2 gives the proofs of Propositions 2.1, 2.3, and 2.5] and of Lemma 2.7. Notice that Proposition 2.5 was proved in 5 and Propositions 2.1 and 2.3 were proved in [5] under Assumptions (H1) and (H2). Thus, it remains to prove Lemma 2.2 and the trace estimate from Proposition 2.4 .

The proof of Lemma 2.2 under Assumption (H0) is given in Section 3 . The proof of Lemma 2.2 under Assumptions (H1) and (H2) is given in Section 8.2

The remaining part of the paper is devoted to the proof of the trace estimate (Proposition 2.4). This result is a refinement of a less precise trace estimate [5, Proposition 5.2] and is obtained from the analysis of a suitable evolution $t \rightarrow U_{n}(t)$ based on Fourier transform, as in [5, Section $6]$. This reduction is presented in Section 4 where we give details which are more involved than in [5]. More precisely, in Section 4 we state Proposition 4.1] which gives $\mathrm{O}\left(n^{-\gamma+\varepsilon}\right)$ estimates for the diagonal entries from the Neumann series expansion of $t \rightarrow U_{n}(t)$ and we show that Proposition 4.1 implies Proposition 2.4. The proof of Proposition 4.1 is given in Section 7 and is based on approximations by oscillatory integrals (Lemma 6.1). In Section 8 we observe that the construction of these approximations was already made in [5, Section 10] and give the proof of the regularity properties claimed in Lemma 6.1.

Concerning the proof of Proposition 4.1] we observe that the principal difficulty consists in the control of oscillatory integrals with phase functions depending on parameters. In particular these phase functions can be identically zero for some values of the parameters but an additional integration allows us to neglect the contribution of these bad cases. More precisely the phase functions appear with a large parameter proportional to $n^{\gamma}$ (see Section 6.2.1) and the results of 5 were based on the fact that the decay of the corresponding oscillatory integrals is of order $n^{-\gamma / 2}$. To obtain the results described in this paper we apply the formula of the asymptotic expansion for oscillatory integrals stated in Lemma 3.1. In Section 7 we investigate the special structure of the main term (of order $n^{-\gamma / 2}$ ) and error terms (of order $n^{-\gamma}$ ) and we manage to control their dependence on parameters by using an auxiliary estimate proved in Section 5 .

1.5. Notations. Throughout the paper, we use the following notations:

- $\mathcal{B}(\mathcal{H})$ is the algebra of linear bounded operators on a Hilbert space $\mathcal{H}$.

- $\mathbb{N}=\{0,1, \ldots\}$ is the set of non-negative integers, $\mathbb{N}^{*}=\{1,2, \ldots\}$ is the set of positive integers.

- $l^{2}(\mathbb{Z})$ is the Hilbert space of square-summable complex sequences $x: \mathbb{Z} \rightarrow \mathbb{C}$ equipped with the scalar product $\langle x, y\rangle=\sum_{k \in \mathbb{Z}} \overline{x(k)} y(k)$ and with the norm $\|x\|_{l^{2}(\mathbb{Z})}:=\sqrt{\langle x, x\rangle}$. 
- $\left\{\mathrm{e}_{k}\right\}_{k \in \mathbb{Z}}$ is the canonical basis of $l^{2}(\mathbb{Z})$, i.e., $\mathrm{e}_{k}(j)=\delta_{k, j}$.

- $H(j, k):=\left\langle\mathrm{e}_{j}, H \mathrm{e}_{k}\right\rangle, j, k \in \mathbb{Z}$ denote the matrix elements of an operator $H$ acting on $l^{2}(\mathbb{Z})$ and defined on its canonical basis.

- $l^{2}\left(\mathbb{N}^{*}\right)$ is the Hilbert space of square-summable complex sequences $x: \mathbb{N}^{*} \rightarrow \mathbb{C}$ equipped with the scalar product $\langle x, y\rangle=\sum_{k \in \mathbb{N}^{*}} \overline{x(k)} y(k)$ and the norm $\|x\|_{l^{2}\left(\mathbb{N}^{*}\right)}:=\sqrt{\langle x, x\rangle}$. It can be identified with the closed subspace of $l^{2}(\mathbb{Z})$ generated by $\left\{\mathrm{e}_{n}\right\}_{n \in \mathbb{N}^{*}}$, i.e., with the subspace defined by the conditions $x(k)=0$ for any $k \leq 0$.

We use specific notations for some operators acting on $l^{2}(\mathbb{Z})$ :

- The shift $S \in \mathcal{B}\left(l^{2}(\mathbb{Z})\right)$ is defined by $(S x)(k)=x(k-1), k \in \mathbb{Z}$. Thus, $S \mathrm{e}_{k}=\mathrm{e}_{k+1}$.

- $\Lambda$ acts on $l^{2}(\mathbb{Z})$ by $(\Lambda x)(k)=k x(k), k \in \mathbb{Z}$ for any $x$ such that $(k x(k))_{k \in \mathbb{Z}} \in l^{2}(\mathbb{Z})$.

- For any $q: \mathbb{Z} \rightarrow \mathbb{C}$ we define the linear operator $q(\Lambda)$ by functional calculus, i.e., $q(\Lambda)$ is the closed operator acting on $l^{2}(\mathbb{Z})$ and characterized by $q(\Lambda) \mathrm{e}_{k}=q(k) \mathrm{e}_{k}, k \in \mathbb{Z}$.

- If $L$ is a self-adjoint operator which is bounded from below with compact resolvent its spectrum is discrete and we denote

$$
\lambda_{1}(L) \leq \cdots \leq \lambda_{k}(L) \leq \lambda_{k+1}(L) \leq \ldots
$$

its eigenvalues, enumerated in non-decreasing order, counting multiplicities.

Finally, we also use the following notations:

- $\mathcal{S}(\mathbb{R})$ denotes the Schwartz class of rapidly decreasing functions $\chi: \mathbb{R} \rightarrow \mathbb{C}$.

- The Fourier transform $\hat{\chi}$ of a function $\chi \in \mathcal{S}(\mathbb{R})$ is defined by

$$
\hat{\chi}(t):=\int_{-\infty}^{\infty} \chi(\lambda) \mathrm{e}^{-\mathrm{i} t \lambda} \frac{\mathrm{d} \lambda}{2 \pi} .
$$

- $\mathbb{T}$ denotes the unit circle $\{z \in \mathbb{C}:|z|=1\}$.

- $\tau_{\omega}: \mathbb{T} \rightarrow \mathbb{T}$, where $\omega \in \mathbb{R}$ denotes the translation $\mathrm{e}^{\mathrm{i} \xi} \mapsto \mathrm{e}^{\mathrm{i}(\xi-\omega)}$.

- $\mathrm{C}^{m}(\mathbb{T}), m=0,1,2, \ldots$ is the space of functions $b: \mathbb{T} \rightarrow \mathbb{C}$ of class $\mathrm{C}^{m}$ equipped with the norm

$$
\|b\|_{\mathrm{C}^{m}(\mathbb{T})}:=\max _{0 \leq k \leq m} \sup _{\xi \in \mathbb{R}}\left|\partial_{\xi}^{k} b\left(\mathrm{e}^{\mathrm{i} \xi}\right)\right| .
$$

Throughout the paper $n \in \mathbb{N}^{*}$ is the large parameter involved in the asymptotics (1.4a) or (1.13a). All error estimates are considered with respect to $n \in \mathbb{N}^{*}$ and some statements will be established only for $n \geq n_{0}$, for some large enough constant $n_{0}$.

\section{Scheme of the proof of Theorems 1.1 and 1.2}

2.1. Plan of Section 2. In Section 2.2 we introduce auxiliary operators $J_{n}, \tilde{V}_{n}$, and $L_{n}$ and state Propositions 2.1 and 2.3 ensuring that $\lambda_{n}(J)$ is well approximated for large $n$ by suitable eigenvalues of $J_{n}$ and $L_{n}$. Moreover, we state Lemma 2.2 which gives the asymptotics of the $n$-th diagonal entry of $\tilde{V}_{n}$. In Section 2.3 we first state Proposition 2.4 which gives a trace estimate for $L_{n}$. To derive from this estimate the asymptotic behavior of eigenvalues of $L_{n}$ we prove Lemma 2.7 which allows us to apply Proposition 2.5. Finally in Section 2.4 we check the implication (1.15).

Remark. Proposition 2.5 was already proved in [5. Propositions 2.1 and 2.3 were proved in $[5$ under assumptions (H1) and (H2). Lemma 2.7 is proved in Section 2.3.3. Lemma 2.2 is proved in Section 3 under assumption (H0) and in Section 8 under assumptions (H1) and (H2). Sections 4 . 5. 6. and 7 are devoted to the proof of Proposition 2.4 (trace estimate).

2.2. Auxiliary operators $J_{n}, \tilde{V}_{n}$, and $L_{n}$. 
2.2.1. Cut-off function. These operators were already introduced in [5]. Their definition depends on the choice of a cut-off function $\theta_{0} \in \mathrm{C}^{\infty}(\mathbb{R})$ such that

$$
\begin{cases}\theta_{0}(t)=1 & \text { if }|t| \leq \frac{1}{6} \\ \theta_{0}(t)=0 & \text { if }|t| \geq \frac{1}{5} \\ 0 \leq \theta_{0}(t) \leq 1 & \text { otherwise }\end{cases}
$$

From now on such a cut-off is fixed and for any $\tau>0$ we denote

$$
\theta_{\tau, n}(s):=\theta_{0}\left(\frac{s-n}{\tau}\right) .
$$

Finally we define $v_{n}, a_{n}: \mathbb{Z} \rightarrow \mathbb{R}$ by

$$
\begin{aligned}
& v_{n}(k):=v(k) \theta_{n, n}(k)^{2}, \\
& a_{n}(k):=(a(n)+(k-n) \delta a(n)) \theta_{2 n, n}(k) .
\end{aligned}
$$

2.2.2. Operators $J_{n}$. As in [5, Section 2.2] we define the self-adjoint operator $J_{n}$ on $l^{2}(\mathbb{Z})$ by

$$
\left(J_{n} x\right)(k)=\left(k+v_{n}(k)\right) x(k)+a_{n}(k) x(k+1)+a_{n}(k-1) x(k-1)
$$

for any $x$ such that $(k x(k))_{k \in \mathbb{Z}} \in l^{2}(\mathbb{Z})$. Using notations of Section 1.5 we can write

$$
J_{n}=\Lambda+v_{n}(\Lambda)+a_{n}(\Lambda) S^{-1}+S a_{n}(\Lambda)
$$

to compare with the similar expression for $J$. Each operator $J_{n}$ is a finite rank perturbation of $\Lambda$, hence its spectrum $\sigma\left(J_{n}\right)$ is discrete and can be written

$$
\sigma\left(J_{n}\right)=\left\{\lambda_{k}\left(J_{n}\right)\right\}_{k \in \mathbb{Z}}
$$

where $\left(\lambda_{k}\left(J_{n}\right)\right)_{k \in \mathbb{Z}}$ denotes the non-decreasing sequence of eigenvalues of $J_{n}$ counted with their multiplicities, well labeled up to translation.

Proposition 2.1. Let $J$ and $J_{n}$ be defined by (1.3) and (2.3), respectively. Assume we are in one of the following two cases:

(a) (H0) is satisfied.

(b) (H1) for some $0<\gamma \leq \frac{1}{2}$ and (H2) are satisfied.

Let $n_{0} \in \mathbb{N}$ be large enough. Then, for any $n \geq n_{0}$ we can enumerate the eigenvalues of $J_{n}$ as in (2.4) so that we have the large $n$ estimate

$$
\lambda_{n}(J)=\lambda_{n}\left(J_{n}\right)+\mathrm{O}\left(n^{3 \gamma-2}\right),
$$

where $\gamma=\frac{1}{2}$ in case (国).

Proof. Case (b) is already proven in [5, Proposition 12.1]. Case (国) requires a new proof, since in that case there is no restriction on $\rho_{N}=|\rho|$.

Let $J_{n}^{+}$be the restriction of $J_{n}$ to the subspace $l^{2}\left(\mathbb{N}^{*}\right)$ which is invariant under $J_{n}$. The operator $J_{n}^{+}$is self-adjoint and bounded from below with compact resolvent. Its spectrum is discrete: $\sigma\left(J_{n}^{+}\right)=\left\{\lambda_{k}\left(J_{n}^{+}\right)\right\}_{k \geq 1}$, where $\lambda_{1}\left(J_{n}^{+}\right) \leq \cdots \leq \lambda_{k}\left(J_{n}^{+}\right) \leq \lambda_{k+1}\left(J_{n}^{+}\right) \leq \ldots$ denote its eigenvalues, enumerated in non-decreasing order, counting multiplicities. Since $J_{n} \mathrm{e}_{k}=k \mathrm{e}_{k}$ for $k \leq 0$ we can write

$$
\sigma\left(J_{n}\right)=\{k \in \mathbb{Z}: k \leq 0\} \cup \sigma\left(J_{n}^{+}\right)=\{k \in \mathbb{Z}: k \leq 0\} \cup\left\{\lambda_{k}\left(J_{n}^{+}\right)\right\}_{k \geq 1} .
$$

Step 1. We will show the estimate

$$
\sup _{n, k \geq 1}\left|\lambda_{k}\left(J_{n}^{+}\right)-k\right|<\infty
$$


Let $J_{0, n}^{+}$denote the operator acting on $l^{2}\left(\mathbb{N}^{*}\right)$ by

$$
\left(J_{0, n}^{+} x\right)(k)=k x(k)+a_{n}(k) x(k+1)+a_{n}(k-1) x(k-1) .
$$

Since $J_{n}^{+}-J_{0, n}^{+}=\operatorname{diag}\left(v_{n}(k)\right)_{k=1}^{\infty}$ with $\left|v_{n}(k)\right| \leq|\rho|$, the min-max principle applies and gives

$$
\sup _{n, k \geq 1}\left|\lambda_{k}\left(J_{n}^{+}\right)-\lambda_{k}\left(J_{0, n}^{+}\right)\right|<\infty .
$$

Hence, to get (2.7) it suffices to show

$$
\sup _{n, k \geq 1}\left|\lambda_{k}\left(J_{0, n}^{+}\right)-k\right|<\infty .
$$

In [5, Proposition 3.1] we proved the large $n$ estimate

$$
\sup _{k \in \mathbb{N}^{*}}\left|\lambda_{k}\left(J_{0, n}^{+}\right)-l_{n}(k)\right|=\mathrm{O}\left(n^{3 \gamma-2}\right)
$$

where

$$
l_{n}(k):=k+a_{n}(k-1)^{2}-a_{n}(k)^{2}, \quad k \geq 1 .
$$

Hence, to obtain (2.9) it suffices to show

$$
\sup _{n, k \geq 1}\left|l_{n}(k)-k\right|<\infty .
$$

To prove (2.12) we denote

$$
a_{1, n}(k):=l_{n}(k)-k=a_{n}(k-1)^{2}-a_{n}(k)^{2} .
$$

By estimate $a_{n}(n-1)^{2}-a_{n}(n)^{2}=a(n-1)^{2}-a(n)^{2}+\mathrm{O}\left(n^{2 \gamma-2}\right)$ from [5, (2.5)] we get

$$
l_{n}(n)=n+a(n-1)^{2}-a(n)^{2}+\mathrm{O}\left(n^{2 \gamma-2}\right) .
$$

In case (国), $\gamma=\frac{1}{2}$ and $a(n)=a_{1} \sqrt{n}$, and thus we obtain

$$
a_{1, n}(n)=l_{n}(n)-n=-a_{1}^{2}+\mathrm{O}\left(n^{-1}\right) .
$$

Moreover, in [5, Section 3.3] we proved the estimate

$$
\left|\delta a_{1, n}(k)\right| \leq C n^{-1},
$$

and thus

$$
\left|a_{1, n}(k)-a_{1, n}(n)\right| \leq|k-n| C n^{-1},
$$

By definition, $a_{1, n}(k)=0$ for any $k \geq 2 n$, and (2.16) ensures

$$
\sup _{n, k \geq 1}\left|a_{1, n}(k)-a_{1, n}(n)\right|<\infty .
$$

Using (2.14) which implies $a_{1, n}=\mathrm{O}(1)$ we finally get (2.12):

$$
\sup _{n, k \geq 1}\left|a_{1, n}(k)\right|<\infty \text {. }
$$

Step 2. Now we will prove that

$$
\sup _{n, k \geq 1}\left|\lambda_{k}\left(\tilde{J}_{n}^{+}\right)-k\right|<\infty .
$$

where the operator $\tilde{J}_{n}^{+}$acts on $l^{2}\left(\mathbb{N}^{*}\right)$ by

$$
\left(\tilde{J}_{n}^{+} x\right)(k)=d_{n}(k) x(k)+\tilde{a}_{n}(k) x(k+1)+\tilde{a}_{n}(k-1) x(k-1),
$$

with

$$
\tilde{a}_{n}(k)= \begin{cases}a(k) & \text { if } n-C_{1}(n+1)^{\gamma} \leq k \leq n+C_{1}(n+1)^{\gamma} \\ a_{n}(k) & \text { otherwise }\end{cases}
$$


and with a large enough constant $C_{1}$. Estimate [5, (12.8)] ensures that

$$
\sup _{k \in \mathbb{N}^{*}}\left|\lambda_{k}\left(\tilde{J}_{n}^{+}\right)-\lambda_{k}\left(J_{n}^{+}\right)\right|=\mathrm{O}\left(n^{3 \gamma-2}\right) .
$$

Thus, (2.7) and (2.19) for $\gamma=1 / 2$ give (2.17).

Step 3. Taking $C_{1}>3$ in the definition (2.18) we have the property

$$
n-3 n^{\gamma} \leq k \leq n+3 n^{\gamma} \Longrightarrow J \mathrm{e}_{k}=\tilde{J}_{n}^{+} \mathrm{e}_{k} .
$$

Then [5, Proposition 12.5] applies and for any $\nu>0$ there exists $n(\nu)$ such that the inequalities

$$
\begin{aligned}
& \operatorname{card}\left\{k \in \mathbb{N}^{*}: \lambda^{\prime}+\lambda^{-\nu}<\lambda_{k}\left(\tilde{J}_{n}^{+}\right) \leq \lambda-\lambda^{-\nu}\right\} \leq \operatorname{card}\left\{k \in \mathbb{N}^{*}: \lambda^{\prime}<\lambda_{k}(J) \leq \lambda\right\}, \\
& \operatorname{card}\left\{k \in \mathbb{N}^{*}: \lambda^{\prime}<\lambda_{k}(J) \leq \lambda\right\} \leq \operatorname{card}\left\{k \in \mathbb{N}^{*}: \lambda^{\prime}-\lambda^{-\nu}<\lambda_{k}\left(\tilde{J}_{n}^{+}\right) \leq \lambda+\lambda^{-\nu}\right\},
\end{aligned}
$$

hold if $n-2 n^{\gamma} \leq \lambda^{\prime}<\lambda \leq n+2 n^{\gamma}$ and $n \geq n(\nu)$. Let $\left\{\Delta_{n}^{\kappa}\right\}_{n \geq 1}, 0<\kappa<1$ be the family of intervals defined by

$$
\Delta_{n}^{\kappa}=\left(n-a_{1}^{2}-\kappa, n-a_{1}^{2}+\kappa\right] .
$$

Using (2.20a) with $\lambda^{\prime}=n-a_{1}^{2}-\frac{3}{4}, \lambda=n-a_{1}^{2}+\frac{3}{4}$, and (2.20b) with $\lambda^{\prime}=n-a_{1}^{2}-\frac{1}{4}, \lambda=n-a_{1}^{2}+\frac{1}{4}$ we get that for some $n_{0}$ and any $n \geq n_{0}$ we have the inequalities

$$
\begin{aligned}
& \operatorname{card}\left\{k \in \mathbb{N}^{*}: \lambda_{k}\left(\tilde{J}_{n}^{+}\right) \in \Delta_{n}^{1 / 2}\right\} \leq \operatorname{card}\left\{k \in \mathbb{N}^{*}: \lambda_{k}(J) \in \Delta_{n}^{3 / 4}\right\}, \\
& \operatorname{card}\left\{k \in \mathbb{N}^{*}: \lambda_{k}\left(\tilde{J}_{n}^{+}\right) \in \Delta_{n}^{1 / 2}\right\} \geq \operatorname{card}\left\{k \in \mathbb{N}^{*}: \lambda_{k}(J) \in \Delta_{n}^{1 / 4}\right\} .
\end{aligned}
$$

Step 4. Yanovich estimate (1.5) gives $\lambda_{n}(J)=n-a_{1}^{2}+\mathrm{o}(1)$. Hence, for any $0<\kappa<1$ and $n(\kappa)$ large enough

$$
n \geq n(\kappa) \Longrightarrow \sigma(J) \cap \Delta_{n}^{\kappa}=\left\{\lambda_{n}(J)\right\} .
$$

Thus we can find $n_{1} \geq n_{0}$ such that the right hand sides of (2.21) are both equal to 1 for $n \geq n_{1}$. Hence, inequalities (2.21) imply

$$
n \geq n_{1} \Longrightarrow \operatorname{card}\left\{k \in \mathbb{N}^{*}: \lambda_{k}\left(\tilde{J}_{n}^{+}\right) \in \Delta_{n}^{1 / 2}\right\}=1 .
$$

Let $k(n)=n+m(n), m(n) \in \mathbb{Z}, n \geq n_{1}$, be the unique $k$ such that $\lambda_{k}\left(\tilde{J}_{n}^{+}\right) \in \Delta_{n}^{1 / 2}$ :

$$
n \geq n_{1} \Longrightarrow \sigma\left(\tilde{J}_{n}^{+}\right) \cap \Delta_{n}^{1 / 2}=\left\{\lambda_{n+m(n)}\left(\tilde{J}_{n}^{+}\right)\right\} .
$$

The eigenvalues $\lambda_{n+m(n)}\left(\tilde{J}_{n}^{+}\right)$are of multiplicity one. Moreover, by (2.17) there exist $\tilde{n}_{0}, m_{0} \in \mathbb{N}^{*}$ large enough such that

$$
n \geq \tilde{n}_{0} \Longrightarrow|m(n)| \leq m_{0} .
$$

Step 5. Using (2.20b) with $\lambda^{\prime}=\lambda_{n}(J)-\lambda_{n}(J)^{-\nu}, \lambda=\lambda_{n}(J)$ we find that, for any fixed $\nu>0$,

$$
\lambda_{n+m(n)}\left(\tilde{J}_{n}^{+}\right)=\lambda_{n}(J)+\mathrm{O}\left(n^{-\nu}\right) .
$$

Then, using (2.19) with $\gamma=\frac{1}{2}$ and (2.25) with $\nu=\frac{1}{2}$ we get

$$
\lambda_{n+m(n)}\left(J_{n}^{+}\right)=\lambda_{n}(J)+\mathrm{O}\left(n^{-1 / 2}\right) .
$$

Let $n \geq \tilde{n}_{1}$ with $\tilde{n}_{1}$ large enough. Then, according to (2.6) and (2.24) we can label the eigenvalues of $J_{n}$ in nondecreasing order, counting multiplicity, so that

$$
\lambda_{n}\left(J_{n}\right)=\lambda_{n+m(n)}\left(J_{n}^{+}\right) .
$$

The proof of (2.5) is then completed by combining (2.26) with (2.27). 
2.2.3. Operators $\tilde{V}_{n}$. As in [5, Section 5.2] we define self-adjoint operators $B_{n}, \tilde{V}_{n} \in \mathcal{B}\left(l^{2}(\mathbb{Z})\right)$ by

$$
\begin{aligned}
B_{n} & :=\mathrm{i}\left(a_{n}(\Lambda) S^{-1}-S a_{n}(\Lambda)\right), \\
\tilde{V}_{n} & :=\mathrm{e}^{\mathrm{i} B_{n}} v_{n}(\Lambda) \mathrm{e}^{-\mathrm{i} B_{n}},
\end{aligned}
$$

where $\left\{a_{n}(k)\right\}_{k \in \mathbb{Z}}$ and $\left\{v_{n}(k)\right\}_{k \in \mathbb{Z}}$ are defined in (2.2), and we denote by $\left\{g_{n}(k)\right\}_{k \in \mathbb{Z}}$ the sequence of diagonal entries of $\tilde{V}_{n}$ :

$$
g_{n}(k):=\tilde{V}_{n}(k, k) \text {. }
$$

Notice that $\tilde{V}_{n}$ and $g_{n}$ depend on both sequences $\{a(k)\}_{k=1}^{\infty},\{v(k)\}_{k=1}^{\infty}$, and on the cut-off $\theta_{0}$.

Lemma 2.2. Let $\tilde{V}_{n}$ be defined by (2.28) and assume we are in one of the following two cases:

(a) (H0) is satisfied.

(b) (H1) and (H2) with $\langle v\rangle=0$ are satisfied.

Then for any $\varepsilon>0$ one has the large $n$ asymptotics

$$
g_{n}(n)=\mathfrak{r}(n)+\mathrm{O}\left(n^{-\gamma+\varepsilon}\right) .
$$

where $\mathfrak{r}(n)$ is given by (1.4b) in case (国) and by (1.13b) in case (b).

Proof. See Section 3.3 for case (a) and Section 8.2 for case (b).

2.2.4. Operators $L_{n}$. As in [5, Section 5.2] we introduce operators $L_{n}$ acting on $l^{2}(\mathbb{Z})$ by

$$
L_{n}:=l_{n}(\Lambda)+\tilde{V}_{n},
$$

where $\tilde{V}_{n}$ is defined by (2.28) and $l_{n}(k)$ is as in (2.11):

$$
l_{n}(k):=k+a_{n}(k-1)^{2}-a_{n}(k)^{2} .
$$

Since $l_{n}(\Lambda)$ is a diagonal operator with discrete spectrum and $\tilde{V}_{n}$ is bounded, the spectrum of $L_{n}$ is discrete and can be written

$$
\sigma\left(L_{n}\right)=\left\{\lambda_{k}\left(L_{n}\right)\right\}_{k \in \mathbb{Z}}
$$

where $\left(\lambda_{k}\left(L_{n}\right)\right)_{k \in \mathbb{Z}}^{\infty}$ denotes the non-decreasing sequence of eigenvalues of $L_{n}$ counted with their multiplicities, well-labeled up to translation. Moreover, the subspace $l^{2}\left(\mathbb{N}^{*}\right)$ is invariant by $B_{n}$, hence by $\tilde{V}_{n}$ and $L_{n}$, and $L_{n} \mathrm{e}_{k}=k \mathrm{e}_{k}$ if $k \leq 0$.

Proposition 2.3. Let $J_{n}$ and $L_{n}$ be defined by (2.3) and (2.31), respectively. We assume we are in one of the following two cases:

(a) (H0) is satisfied.

(b) (H1) for some $0<\gamma \leq \frac{1}{2}$ and (H2) are satisfied.

Then, the eigenvalues of $L_{n}$ can be enumerated in nondecreasing order, counting multiplicity, so that one has the large $n$ estimate

$$
\lambda_{n}\left(J_{n}\right)=\lambda_{n}\left(L_{n}\right)+\mathrm{O}\left(n^{3 \gamma-2}\right),
$$

where $\gamma=\frac{1}{2}$ in case (国).

Proof. We indeed have $\left\|J_{n}-L_{n}\right\|=\mathrm{O}\left(n^{3 \gamma-2}\right)$, see [5, proof of Proposition 5.1]. Hence, we can enumerate the eigenvalues of $L_{n}$ in (2.32) so that

$$
\sup _{k \in \mathbb{Z}}\left|\lambda_{k}\left(J_{n}\right)-\lambda_{k}\left(L_{n}\right)\right|=\mathrm{O}\left(n^{3 \gamma-2}\right) .
$$

Summary. In both cases (国) and (b) Propositions 2.1 and 2.3 imply the large $n$ estimate $\lambda_{n}(J)=$ $\lambda_{n}\left(L_{n}\right)+\mathrm{O}\left(n^{3 \gamma-2}\right)$. Since $\gamma \leq \frac{1}{2}$ we also get

$$
\lambda_{n}(J)=\lambda_{n}\left(L_{n}\right)+\mathrm{O}\left(n^{-\gamma}\right) .
$$




\subsection{Trace estimate and its consequences.}

2.3.1. The trace estimate. Further on we denote

$$
\tilde{l}_{n}(k):=l_{n}(k)+g_{n}(k)
$$

where $l_{n}$ and $g_{n}$ are given by (2.31b) and (2.29), respectively. Then, for $\chi \in \mathcal{S}(\mathbb{R})$ we consider

$$
\tilde{\mathcal{G}}_{n}^{0}:=\sum_{j \in \mathbb{Z}}\left(\chi\left(\lambda_{n+j}\left(L_{n}\right)-l_{n}(n)\right)-\chi\left(\tilde{l}_{n}(n+j)-l_{n}(n)\right)\right)
$$

with $L_{n}$ as in (2.31a). Writing $k=j+n$ in (2.36a) we get the expression

$$
\tilde{\mathcal{G}}_{n}^{0}=\sum_{k \in \mathbb{Z}}\left(\chi\left(\lambda_{k}\left(L_{n}\right)-l_{n}(n)\right)-\chi\left(\tilde{l}_{n}(k)-l_{n}(n)\right)\right) .
$$

Introducing the diagonal operators

$$
\begin{aligned}
L_{0, n} & :=l_{n}(\Lambda), \\
\tilde{L}_{0, n} & :=\tilde{l}_{n}(\Lambda)=L_{0, n}+g_{n}(\Lambda)
\end{aligned}
$$

we see that the r.h.s. of (2.36b) is the trace of an operator:

$$
\tilde{\mathcal{G}}_{n}^{0}=\operatorname{tr}\left(\chi\left(L_{n}-l_{n}(n)\right)-\chi\left(\tilde{L}_{0, n}-l_{n}(n)\right)\right) .
$$

Notice that $\tilde{\mathcal{G}}_{n}^{0}$ depends on $\chi \in \mathcal{S}(\mathbb{R})$. It also depends on $\{a(k)\}_{k=1}^{\infty},\{v(k)\}_{k=1}^{\infty}$, and $\theta_{0}$.

Warning. This trace $\tilde{\mathcal{G}}_{n}^{0}$ differs from the trace $\mathcal{G}_{n}^{0}$ considered in [5, formulas (5.10)], that uses $L_{0, n}$ instead of $\tilde{L}_{0, n}$, and thus does not involve $g_{n}$.

Proposition 2.4 (trace estimate). Let $\tilde{\mathcal{G}}_{n}^{0}$ be the trace defined by (2.36a) under the additional assumption that $\chi \in \mathcal{S}(\mathbb{R})$ has Fourier transform with compact support. Then, under assumption $(\mathrm{H} 0)$ or assumptions $(\mathrm{H} 1)$ and $(\mathrm{H} 2)$ and for any $\varepsilon>0$ one has the large $n$ estimate

$$
\tilde{\mathcal{G}}_{n}^{0}=\mathrm{O}\left(n^{-\gamma+\varepsilon}\right) .
$$

Proof. See Section 4.3 where the proof is reduced to that of Proposition 4.1. See also Section 7 where the proof of Proposition 4.1 is given.

2.3.2. Comparison of the asymptotic behavior of two sequences. As before $\left(l_{n}(k)\right)_{k \in \mathbb{Z}}$ is defined by (2.31b) where $\left(a_{n}(k)\right)_{k \in \mathbb{Z}}$ is given by (2.2b) under assumption (H1).

Proposition 2.5. Let $\left(l_{n}(k)\right)_{k \in \mathbb{Z}}$ be defined by (2.31b) under assumption $(\mathrm{H} 1)$ for some $0<\gamma \leq$ $\frac{1}{2}$. For each $j \in \mathbb{Z}$ let $\left(r_{n}^{0}(j)\right)_{n=1}^{\infty}$ and $\left(r_{n}^{1}(j)\right)_{n=1}^{\infty}$ be real valued sequences such that

$$
\sup _{j \in \mathbb{Z}, n \geq 1}\left(\left|r_{n}^{0}(j)\right|+\left|r_{n}^{1}(j)\right|\right)<\infty .
$$

Assume they also satisfy

$$
\begin{aligned}
& \sup _{|j| \leq n \gamma_{0}}\left|r_{n}^{i}(j+N)-r_{n}^{i}(j)\right| \leq C n^{\gamma-1} \quad(i=0,1), \\
& \sup _{n>n_{0}} \sup _{|j| \leq n \gamma_{0}}\left(\left|r_{n}^{0}(j)\right|+\left|r_{n}^{1}(j)\right|\right) \leq \rho^{\prime}
\end{aligned}
$$

for some $\gamma_{0}>0, n_{0} \in \mathbb{N}$, and $\rho^{\prime}>0$ such that

$$
\rho^{\prime}< \begin{cases}\frac{1}{2} & \text { when } N=2, \\ \frac{1}{\pi \sqrt{N}} & \text { when } N \geq 3 .\end{cases}
$$


Assume moreover that for some $\varepsilon>0$

$$
\sum_{j \in \mathbb{Z}}\left(\chi\left(l_{n}(n+j)+r_{n}^{1}(j)-l_{n}(n)\right)-\chi\left(l_{n}(n+j)+r_{n}^{0}(j)-l_{n}(n)\right)\right)=\mathrm{O}\left(n^{-\gamma+\varepsilon}\right)
$$

holds for any $\chi \in \mathcal{S}(\mathbb{R})$ whose Fourier transform has compact support. Then

$$
r_{n}^{1}(0)=r_{n}^{0}(0)+\mathrm{O}\left(n^{-\gamma+\varepsilon}\right) .
$$

Proof. It suffices to adapt the proof of [5] Proposition 11.1] as follows. Remove the first two lines, define $G_{n}^{\chi}$ as the l.h.s. of (2.42) and replace the error terms $\mathrm{O}\left(n^{-\gamma / 2} \ln n\right)$ by $\mathrm{O}\left(n^{-\gamma+\varepsilon}\right)$.

2.3.3. Application of Proposition 2.5. We will apply Proposition 2.5 to the case where the two sequences $\left(r_{n}^{0}(j)\right)_{n=1}^{\infty}$ and $\left(r_{n}^{1}(j)\right)_{n=1}^{\infty}$ are given by

$$
\begin{aligned}
r_{n}^{0}(j) & :=g_{n}(n+j), \\
r_{n}^{1}(j) & :=\lambda_{n+j}\left(L_{n}\right)-l_{n}(n+j) .
\end{aligned}
$$

Proposition 2.6. Let $L_{n}$ and $\tilde{l}_{n}(k)$ be given by (2.31a) and (2.35), respectively. We assume we are in one of the following two cases:

(a) (H0) is satisfied, $\gamma=\frac{1}{2}$.

(b) (H1) for some $0<\gamma \leq \frac{1}{2}$ and (H2) are satisfied.

Then, for any $\varepsilon>0$ one has the large $n$ estimate

$$
\lambda_{n}\left(L_{n}\right)=\tilde{l}_{n}(n)+\mathrm{O}\left(n^{-\gamma+\varepsilon}\right)
$$

Proof. We first show that Proposition 2.5 applies to the case where $r_{n}^{0}$ and $r_{n}^{1}$ are given by (2.44). In this case the l.h.s. of (2.42) is $\tilde{\mathcal{G}}_{n}^{0}$ as defined by (2.36a) and thus the trace estimate (2.39) in Proposition 2.4 says that assumption (2.42) is satisfied for any $\varepsilon>0$ and any $\chi \in \mathcal{S}(\mathbb{R})$ whose Fourier transform has compact support. That the conditions (2.40) are also satisfied is proven in Lemma 2.7 below. Thus, Proposition 2.5 applies and the assertion (2.43) is exactly (2.45) since in that case $r_{n}^{1}(0)-r_{n}^{0}(0)=\lambda_{n}\left(L_{n}\right)-\tilde{l}_{n}(n)$.

Lemma 2.7. The sequences $r_{n}^{0}$ and $r_{n}^{1}$ given by (2.44) satisfy the conditions (2.40) in each of the following two cases:

(a) (H0) is satisfied, $N=2, \gamma=\frac{1}{2}, \gamma_{0}=\frac{1}{4}$, any $0<\rho^{\prime}<\frac{1}{2}$, and $n_{0} \in \mathbb{N}$ large enough.

(b) (H1) and (H2) are satisfied, $0<\gamma \leq \frac{1}{2}, \gamma_{0}=\gamma$, any $\rho^{\prime}>\rho_{N}$ satisfying (2.41), and $n_{0} \in \mathbb{N}$ large enough.

Proof. We first prove (2.40b).

Step 1 (estimate of $r_{n}^{0}(j)$ in cases (或) and ( $(\underline{b})$ ). First note that $r_{n}^{0}(j)=g_{n}(n+j)$. Then, taking $t_{1}=0$ and $j+n$ instead of $j$ in [5, Lemma 6.3 (i)] we get

$$
\sup _{|j| \leq n^{\gamma}}\left|r_{n}^{0}(j)\right|=\sup _{|j| \leq n^{\gamma}}\left|g_{n}(n+j)\right|=\mathrm{O}\left(n^{-\gamma / 2}\right) .
$$

We indeed have the relation $g_{1, n, j}(0)=\mathrm{i} g_{n}(j)$ with $g_{1, n, j}$ defined in [5, (6.16)].

Step 2 (estimate of $r_{n}^{1}(j)$ in case (国)). It suffices to show

$$
\sup _{|j| \leq n^{1 / 4}}\left|r_{n}^{1}(j)\right|=\mathrm{O}\left(n^{-1 / 16}\right) \text {. }
$$

To obtain (2.47) we introduce the quantity

$$
\tilde{r}_{n}^{1}(j):=\lambda_{n+j}(J)-\left(n+j-a_{1}^{2}\right)
$$


and observe that the Yanovich estimate (1.5) ensures

$$
\sup _{|j| \leq n^{1 / 4}}\left|\tilde{r}_{n}^{1}(j)\right|=\mathrm{O}\left(n^{-1 / 16}\right) .
$$

Due to (2.49) to complete the proof of (2.47) it suffices to show the estimate

$$
\sup _{|j| \leq n^{1 / 4}}\left|r_{n}^{1}(j)-\tilde{r}_{n}^{1}(j)\right|=\mathrm{O}\left(n^{-1 / 2}\right) .
$$

In order to get (2.50) we first show the estimate

$$
\sup _{|j| \leq n^{1 / 4}}\left|\lambda_{n}(J)-\lambda_{n}\left(J_{n-j}\right)\right|=\mathrm{O}\left(n^{-1 / 2}\right) .
$$

For this purpose we observe that the proof given in [5, Section 12.5] still holds if $\tilde{J}_{n}^{+}$is replaced by $\tilde{J}_{n-j}^{+}$with $|j| \leq n^{1 / 4}$ and all estimates are uniform with respect to $j$. Hence we can replace $J_{n}$ by $J_{n-j}$ in (2.5), and (2.51) is proved. Moreover, replacing $n$ by $n+j$ we can write (2.51) in the form

$$
\sup _{|j| \leq n^{1 / 4}}\left|\lambda_{n+j}(J)-\lambda_{n+j}\left(J_{n}\right)\right|=\mathrm{O}\left(n^{-1 / 2}\right) .
$$

Then we complete the proof of (2.50) by showing

$$
\sup _{|j| \leq n^{1 / 4}}\left|l_{n}(n+j)-\left(n+j-a_{1}^{2}\right)\right|=\mathrm{O}\left(n^{-3 / 4}\right) .
$$

In order to show (2.53) we consider $a_{1, n}(k)=l_{n}(k)-k$ as in (2.13). Using (2.15) we get

$$
\left|l_{n}(n+j)-l_{n}(n)-j\right|=\left|a_{1, n}(n+j)-a_{1, n}(n)\right| \leq|j| C n^{-1},
$$

hence

$$
\sup _{|j| \leq n^{1 / 4}}\left|l_{n}(n+j)-l_{n}(n)-j\right|=\mathrm{O}\left(n^{-3 / 4}\right),
$$

and (2.53) follows by using (2.14) in the last estimate.

Step 3 (estimate of $r_{n}^{1}(j)$ in case (b) ). In [5, Section 11.3] where $r_{n}^{1}$ is denoted by $r_{n}$ (see [5. (11.16)]) we have shown the estimate

$$
\sup _{j \in \mathbb{Z}}\left|r_{n}^{1}(j)\right| \leq \rho_{N}+C_{1} n^{3 \gamma-2} .
$$

We indeed have the relation $g_{1, n, j}(0)=\mathrm{i} g_{n}(j)$ with $g_{1, n, j}$ defined in [5, (6.16)]. Using (2.54), (2.46) and taking $\gamma_{0}=\gamma$ we can estimate the l.h.s. of (2.40b) by $\rho_{N}+C_{1} n^{3 \gamma-2}+C_{2} n^{-\gamma / 2}$. Moreover, by assumption ( $\mathrm{H} 2 \mathrm{C})$ on $\rho_{N}$ we can choose $\rho^{\prime}>\rho_{N}$ satisfying (2.41). We conclude that (2.40b) holds if $n_{0}$ satisfies $C_{1} n_{0}^{3 \gamma-2}+C_{2} n_{0}^{-\gamma / 2} \leq \rho^{\prime}-\rho_{N}$, and that is possible since $0<\gamma<\frac{2}{3}$.

We now prove (2.40a).

Step 4 (proof of (2.40a) for $i=0$ ). Since $g_{n}(k+N)=\left\langle\mathrm{e}_{k}, S^{-N} \tilde{V}_{n} S^{N} \mathrm{e}_{k}\right\rangle$ it suffices to prove

$$
\left\|S^{-N} \tilde{V}_{n} S^{N}-\tilde{V}_{n}\right\|=\mathrm{O}\left(n^{\gamma-1}\right) .
$$

In order to show (2.55) we first observe that $S^{-N} v(\Lambda) S^{N}=v(\Lambda+N)=v(\Lambda)$ and $\| S^{-N} \theta_{n, n}(\Lambda) S^{N}$ $\theta_{n, n}(\Lambda)\|=\| \theta_{0}((\Lambda+N) / n-I)-\theta_{0}(\Lambda / n-I) \|=\mathrm{O}\left(n^{-1}\right)$ ensure

$$
\left\|S^{-N} v_{n}(\Lambda) S^{N}-v_{n}(\Lambda)\right\|=\mathrm{O}\left(n^{\gamma-1}\right) .
$$

Similarly, $\left\|S^{-N} a_{n}(\Lambda) S^{N}-a_{n}(\Lambda)\right\|=\left\|a_{n}(\Lambda+N)-a_{n}(\Lambda)\right\|=\mathrm{O}\left(n^{\gamma-1}\right)$ implies

$$
\left\|S^{-N} \mathrm{e}^{ \pm \mathrm{i} B_{n}} S^{N}-\mathrm{e}^{ \pm \mathrm{i} B_{n}}\right\|=\mathrm{O}\left(n^{\gamma-1}\right)
$$

and (2.55) follows from (2.56) and (2.57). 
Step 5 (proof of (2.40a) for $i=1$ in case (b)). In [5, Section 11.3] where $r_{n}^{1}$ is denoted by $r_{n}$ we already checked that (2.40a) holds for $i=1$.

Step 6 (proof of (2.40a) for $i=1$ in case (国)). We observe that if $0<\kappa<1$ then combining (2.22) and (2.52) we can choose $n_{\kappa}$ large enough to ensure

$$
n \geq n_{\kappa} \text { and }|j| \leq n^{1 / 4} \Longrightarrow \sigma\left(J_{n}\right) \cap\left(n+j-a_{1}^{2}-\kappa, n+j-a_{1}^{2}+\kappa\right]=\left\{\lambda_{n+j}\left(J_{n}\right)\right\}
$$

and (2.58) allows us to obtain (2.40a) for $i=1$ following the proof given in [5, Section 11.3].

2.4. Proof of Theorems $\mathbf{1 . 1}$ and 1.2. Recall that Propositions 2.1 and 2.3 give the large $n$ estimate $\lambda_{n}(J)=\lambda_{n}\left(L_{n}\right)+\mathrm{O}\left(n^{-\gamma}\right)$. Combining this estimate (2.34) with estimate (2.45) of $\lambda_{n}\left(L_{n}\right)$ in Proposition 2.6 we obtain

$$
\lambda_{n}(J)=\tilde{l}_{n}(n)+\mathrm{O}\left(n^{-\gamma+\varepsilon}\right)
$$

for any $\varepsilon>0$. The desired estimates (1.4a) and (1.13a) follow from (2.59), using the estimate (2.30) of $g_{n}(n)$, and from

$$
l_{n}(n)-n=a_{n}(n-1)^{2}-a_{n}(n)^{2}=a(n-1)^{2}-a(n)^{2}+\mathrm{O}\left(n^{2 \gamma-2}\right)
$$

whose last estimate comes from [5, Section 2.3, (2.5)].

\section{Proof of Lemma 2.2 (a)}

In section 3.1 we prove a stationary phase formula for some type of oscillatory integral (Lemma 3.11). Then we assume that the diagonal and off-diagonal entries of $J$ satisfy $(\mathrm{H} 1)$ and $d(k)=$ $k+v(k)$ with $v(k)=(-1)^{k} \rho$. In section 3.2 we prove an approximation result of $g_{n}(n)$ by an oscillatory integral of the above type (Lemma 3.2). Finally, in section 3.3 we derive the asymptotics (2.30) of $g_{n}(n)$ :

$$
\left.\begin{array}{l}
\text { Lemma } 3.1 \\
\text { Lemma } 3.2
\end{array}\right\} \Longrightarrow \text { Lemma } 2.2 \text { (a). }
$$

\subsection{Stationary phase formula.}

Lemma 3.1. For $b \in \mathrm{C}^{2}(\mathbb{T}), \eta_{0} \in \mathbb{R}$ and $\mu>0$ we consider the oscillatory integral

$$
\mathcal{J}\left(b, \mu, \eta_{0}\right):=\int_{0}^{2 \pi} \mathrm{e}^{\mathrm{i} \mu \cos \left(\eta-\eta_{0}\right)} b\left(\mathrm{e}^{\mathrm{i} \eta}\right) \frac{\mathrm{d} \eta}{2 \pi} .
$$

If we write

$$
\mathcal{J}\left(b, \mu, \eta_{0}\right)=\sum_{\kappa= \pm 1} \frac{\mathrm{e}^{\mathrm{i} \kappa(\mu-\pi / 4)}}{\sqrt{2 \pi \mu}} b\left(\kappa \mathrm{e}^{\mathrm{i} \eta_{0}}\right)+r_{b}\left(\mu, \eta_{0}\right)
$$

then the remainder $r_{b}\left(\mu, \eta_{0}\right)$ satisfies the estimate

$$
\left|r_{b}\left(\mu, \eta_{0}\right)\right| \leq \frac{C_{0}}{\mu}\|b\|_{\mathrm{C}^{2}(\mathbb{T})}
$$

for some constant $C_{0}$.

Proof. Let $\chi \in \mathrm{C}^{\infty}(\mathbb{T})$ be such that $\chi\left(\mathrm{e}^{\mathrm{i}\left(\xi+\eta_{0}\right)}\right)=1$ if $|\xi| \leq \pi / 4$ and $\chi\left(\mathrm{e}^{\mathrm{i}\left(\xi+\eta_{0}\right)}\right)=0$ if $3 \pi / 4 \leq$ $|\xi| \leq 5 \pi / 4$. Since the integrands are $2 \pi$-periodic the change of variable $\eta=\xi+\eta_{0}$ gives

$$
\mathcal{J}\left(\chi b, \mu, \eta_{0}\right)=\int_{0}^{2 \pi} \mathrm{e}^{\mathrm{i} \mu \cos \xi}(\chi b)\left(\mathrm{e}^{\mathrm{i}\left(\xi+\eta_{0}\right)}\right) \frac{\mathrm{d} \xi}{2 \pi} .
$$

Denoting $b_{+}(\xi):=b\left(\mathrm{e}^{\mathrm{i}\left(\xi+\eta_{0}\right)}\right)$ and $\chi_{+}(\xi):=\chi\left(\mathrm{e}^{\mathrm{i}\left(\xi+\eta_{0}\right)}\right)$, we can express

$$
\mathcal{J}\left(\chi b, \mu, \eta_{0}\right)=\int_{-\pi}^{\pi} \mathrm{e}^{\mathrm{i} \mu \cos \xi}\left(\chi_{+} b_{+}\right)(\xi) \frac{\mathrm{d} \xi}{2 \pi} .
$$


Let $b_{-}(\xi):=b\left(\mathrm{e}^{\mathrm{i}\left(\xi+\eta_{0}-\pi\right)}\right)$ and $\chi_{-}(\xi):=1-\chi\left(\mathrm{e}^{\mathrm{i}\left(\xi+\eta_{0}-\pi\right)}\right)$. We still have $\chi_{-}(\xi)=1$ if $|\xi| \leq \pi / 4$ and $\chi_{-}(\xi)=0$ if $3 \pi / 4 \leq|\xi| \leq 5 \pi / 4$. Then the change of variable $\eta=\xi+\eta_{0}-\pi$ gives

$$
\mathcal{J}\left((1-\chi) b, \mu, \eta_{0}\right)=\int_{-\pi}^{\pi} \mathrm{e}^{-\mathrm{i} \mu \cos \xi}\left(\chi_{-} b_{-}\right)(\xi) \frac{\mathrm{d} \xi}{2 \pi} .
$$

We have $[-\pi, \pi] \cap \operatorname{supp} \chi_{ \pm} \subset\left[-\frac{3 \pi}{4}, \frac{3 \pi}{4}\right]$. Next we observe that $|\xi| \leq 3 \pi / 4$ allows us to write

$$
b_{ \pm}(\xi)=b_{ \pm}(0)+q_{ \pm}(\xi) \xi=b_{ \pm}(0)+\tilde{q}_{ \pm}(\xi) \sin \xi
$$

with $\tilde{q}_{ \pm}(\xi):=q_{ \pm}(\xi) \frac{\xi}{\sin \xi}$. Moreover, $\chi_{ \pm}(0)=1$ and the standard stationary phase formula ensures

$$
\left|\int_{-\pi}^{\pi} \mathrm{e}^{ \pm \mathrm{i} \mu \cos \xi} \chi_{ \pm}(\xi) b_{ \pm}(0) \mathrm{d} \xi-\frac{\mathrm{e}^{ \pm \mathrm{i}(\mu-\pi / 4)}}{\sqrt{2 \pi \mu}} b_{ \pm}(0)\right| \leq \frac{C_{\chi_{ \pm}}}{\mu}\left|b_{ \pm}(0)\right| .
$$

Then writing $\mathrm{e}^{ \pm \mathrm{i} \mu \cos \xi} \sin \xi=\frac{ \pm \mathrm{i}}{\mu} \partial_{\xi} \mathrm{e}^{ \pm \mathrm{i} \mu \cos \xi}$ and integrating by parts we obtain

$$
\int_{-\pi}^{\pi} \tilde{q}_{ \pm}(\xi) \sin \xi \mathrm{e}^{ \pm \mathrm{i} \mu \cos (\xi)} \chi_{ \pm}(\xi) \mathrm{d} \xi=\frac{ \pm \mathrm{i}}{\mu} \int_{-\pi}^{\pi} \mathrm{e}^{ \pm \mathrm{i} \mu \cos \xi} \partial_{\xi}\left(\left(\tilde{q}_{ \pm} \chi_{ \pm}\right)(\xi)\right) \mathrm{d} \xi
$$

Since the absolute value of the right hand side of (3.3) can be estimated by $\frac{C_{1}}{\mu}\left\|b_{ \pm}\right\|_{\mathrm{C}^{2}(\mathbb{R})}$ the proof is complete.

3.2. Approximation of $\boldsymbol{g}_{\boldsymbol{n}}(\boldsymbol{n})$ by an oscillatory integral. Recall that $g_{n}(k), k \in \mathbb{Z}$ is defined in (2.29) as the $k$-th diagonal entry of $\tilde{V}_{n}:=\mathrm{e}^{\mathrm{i} B_{n}} v_{n}(\Lambda) \mathrm{e}^{-\mathrm{i} B_{n}}$. We define $\tilde{\varphi}_{n}: \mathbb{Z} \times \mathbb{T} \rightarrow \mathbb{C}$ by

$$
\tilde{\varphi}_{n}\left(k, \mathrm{e}^{\mathrm{i} \xi}\right):=-4(a(n)+(k-n) \delta a(n))(\sin \xi+\delta a(n) \sin 2 \xi)
$$

Lemma 3.2. We assume that the diagonal entries of $J$ are of the form $d(k)=k+(-1)^{k} \rho$ and the off-diagonal entries a( $k$ ) satisfy (H1) for some $0<\gamma \leq \frac{1}{2}$. Let $g_{n}(k), k \in \mathbb{Z}$ be defined by (2.29). If $\mathfrak{g}_{n}(k), k \in \mathbb{Z}$ is defined by

$$
\mathfrak{g}_{n}(k):=(-1)^{k} \rho \int_{0}^{2 \pi} \mathrm{e}^{\mathrm{i} \tilde{\varphi}_{n}\left(k, \mathrm{e}^{\mathrm{i} \xi}\right)} \frac{\mathrm{d} \xi}{2 \pi},
$$

with $\tilde{\varphi}_{n}$ as above, then

$$
\sup _{|k-n| \leq n^{\gamma}}\left|g_{n}(k)-\mathfrak{g}_{n}(k)\right|=\mathrm{O}\left(n^{-\gamma} \ln n\right) .
$$

Proof. As in [5] we denote

$$
\Theta_{n}:=\theta_{n, n}(\Lambda)=\theta_{0}(\Lambda / n-I)
$$

Then it is easy to check the estimate $\left\|\left[B_{n}, \Theta_{n}\right]\right\|=\mathrm{O}\left(n^{\gamma-1}\right)$. Writing

$$
\left[\mathrm{e}^{ \pm \mathrm{i} B_{n}}, \Theta_{n}\right]=\int_{0}^{1} \mathrm{e}^{ \pm \mathrm{i} t B_{n}}\left[ \pm \mathrm{i} B_{n}, \Theta_{n}\right] \mathrm{e}^{ \pm \mathrm{i}(1-t) B_{n}} \mathrm{~d} t
$$

we deduce

$$
\left\|\left[\mathrm{e}^{ \pm \mathrm{i} B_{n}}, \Theta_{n}\right]\right\|=\mathrm{O}\left(n^{\gamma-1}\right) .
$$

We recall that $\tilde{V}_{n}=\mathrm{e}^{\mathrm{i} B_{n}} \Theta_{n} v(\Lambda) \Theta_{n} \mathrm{e}^{-\mathrm{i} B_{n}}$ and observe that (3.6) ensures

$$
\left\|\tilde{V}_{n}-\Theta_{n} \mathrm{e}^{\mathrm{i} B_{n}} v(\Lambda) \mathrm{e}^{-\mathrm{i} B_{n}} \Theta_{n}\right\|=\mathrm{O}\left(n^{\gamma-1}\right) .
$$

Further on we assume that $|k-n| \leq n^{\gamma}$. Using $v(\Lambda)=\rho \mathrm{e}^{\mathrm{i} \pi \Lambda}$ and (3.7) we obtain

$$
g_{n}(k)=\rho\left\langle\mathrm{e}_{k}, \mathrm{e}^{\mathrm{i} B_{n}} \mathrm{e}^{\mathrm{i} \pi \Lambda} \mathrm{e}^{-\mathrm{i} B_{n}} \mathrm{e}_{k}\right\rangle+\mathrm{O}\left(n^{\gamma-1}\right) .
$$

However, $\mathrm{e}^{\mathrm{i} \pi \Lambda} S^{ \pm 1} \mathrm{e}^{-\mathrm{i} \pi \Lambda}=-S^{ \pm 1}$ implies $\mathrm{e}^{\mathrm{i} \pi \Lambda} B_{n} \mathrm{e}^{-\mathrm{i} \pi \Lambda}=-B_{n}$, hence

$$
\mathrm{e}^{\mathrm{i} B_{n}} \mathrm{e}^{\mathrm{i} \pi \Lambda}=\mathrm{e}^{\mathrm{i} \pi \Lambda} \mathrm{e}^{-\mathrm{i} B_{n}} .
$$


Using (3.8) and (3.9) we obtain

$$
g_{n}(k)=\rho \mathrm{e}^{\mathrm{i} \pi k}\left\langle\mathrm{e}_{k}, \mathrm{e}^{-2 \mathrm{i} B_{n}} \mathrm{e}_{k}\right\rangle+\mathrm{O}\left(n^{\gamma-1}\right)
$$

Let $Q_{n}^{t}$ be the operators introduced in [5, proof of Proposition 8.1]. For $t \in[-2,2]$ and $k \in \mathbb{N}^{*}$ we have

$$
\left|\left\langle\mathrm{e}_{k}, \Theta_{n} \mathrm{e}^{\mathrm{i} t B_{n}} \mathrm{e}_{k}\right\rangle-\left\langle\mathrm{e}_{k}, Q_{n}^{t} \mathrm{e}_{k}\right\rangle\right| \leq\left\|\Theta_{n} \mathrm{e}^{\mathrm{i} t B_{n}}-Q_{n}^{t}\right\| \leq C n^{\gamma-1} \ln n .
$$

Moreover,

$$
Q_{n}^{t}(k, k)=\theta_{n}(k) \int_{0}^{2 \pi} \mathrm{e}^{\mathrm{i} \tilde{\psi}_{n}^{t}\left(k, \mathrm{e}^{\mathrm{i} \xi}\right)} \frac{\mathrm{d} \xi}{2 \pi}
$$

where $\tilde{\psi}_{n}^{t}$ is given by [5, (8.5a)]. Observe now that $\tilde{\varphi}_{n}$ given by (3.4) coincides with $\tilde{\psi}_{n}^{t}$ for $t=-2$. Thus, to complete the proof of (3.5b) it suffices to use (3.10) and (3.11) with $t=-2$.

3.3. End of the proof of Lemma 2.2 (a). By definitions (3.5a) and (3.4) we have

$$
\mathfrak{g}_{n}(n)=(-1)^{n} \rho \int_{0}^{2 \pi} \mathrm{e}^{-4 \mathrm{i} a(n) \sin \xi} b_{n}\left(\mathrm{e}^{\mathrm{i} \xi}\right) \frac{\mathrm{d} \xi}{2 \pi},
$$

where

$$
b_{n}\left(\mathrm{e}^{\mathrm{i} \xi}\right):=\mathrm{e}^{-4 \mathrm{i} a(n) \delta a(n) \sin 2 \xi} .
$$

Thus, using notation (3.1) we can write

$$
\mathfrak{g}_{n}(n)=(-1)^{n} \rho \mathcal{J}\left(b_{n}, 4 a(n),-\pi / 2\right) .
$$

By (H1a) and (H1b) with $0<\gamma \leq \frac{1}{2}$ we have $\left\|b_{n}\right\|_{\mathrm{C}^{2}(\mathbb{T})}=\mathrm{O}(1)$. We also have $\mathrm{O}\left(a(n)^{-1}\right)=$ $\mathrm{O}\left(n^{-\gamma}\right)$ by (H1a) and $b_{n}\left( \pm \mathrm{e}^{-\mathrm{i} \pi / 2}\right)=1$. Then the stationary phase formula of Lemma 3.1 gives

$$
\begin{aligned}
\mathfrak{g}_{n}(n) & =(-1)^{n} \rho \sum_{\kappa= \pm 1} \frac{\mathrm{e}^{\mathrm{i} \kappa(4 a(n)-\pi / 4)}}{2 \sqrt{2 \pi a(n)}} b_{n}\left(\kappa \mathrm{e}^{-\mathrm{i} \pi / 2}\right)+\mathrm{O}\left(a(n)^{-1}\right) \\
& =(-1)^{n} \rho \frac{\cos (4 a(n)-\pi / 4)}{\sqrt{2 \pi a(n)}}+\mathrm{O}\left(n^{-\gamma}\right) \\
& =\mathfrak{r}(n)+\mathrm{O}\left(n^{-\gamma}\right)
\end{aligned}
$$

with $\mathfrak{r}(n)$ as in (1.4b). This estimate, together with the estimate

$$
\left|g_{n}(n)-\mathfrak{g}_{n}(n)\right|=\mathrm{O}\left(n^{-\gamma} \ln n\right)
$$

from Lemma 3.2. gives $g_{n}(n)=\mathfrak{r}(n)+\mathrm{O}\left(n^{-\gamma+\varepsilon}\right)$ for any $\varepsilon>0$, i.e. estimate (2.30), in case (a).

\section{Trace estimate: A First Reduction}

In this section we reduce the proof of Proposition 2.4 to that of Proposition 4.1 using the representation of functions of operators by means of Fourier transform. This representation allows us to investigate the quantity $\tilde{\mathcal{G}}_{n}^{0}$ using the Neumann series of

$$
U_{n}(t):=\mathrm{e}^{-\mathrm{i} t l_{n}(\Lambda)} \mathrm{e}^{\mathrm{i} t L_{n}} \quad(t \in \mathbb{R}) .
$$

The proof of this reduction is based on Lemma 4.2 which develops our ideas from [5, Section 6] and allows us to conclude in Section 4.3

$$
\left.\begin{array}{l}
\text { Proposition 4.1 } \\
\text { Lemma } 4.2
\end{array}\right\} \Longrightarrow \text { Proposition 2.4 }
$$


4.1. Properties of the evolution $\boldsymbol{U}_{\boldsymbol{n}}(\boldsymbol{t})$. Using (4.1) and $L_{n}-l_{n}(\Lambda)=L_{n}-L_{0, n}=\tilde{V}_{n}$, we get

$$
-\mathrm{i} \partial_{t} U_{n}(t)=H_{n}(t) U_{n}(t)
$$

where

$$
H_{n}(t):=\mathrm{e}^{-\mathrm{i} t L_{0, n}} \tilde{V}_{n} \mathrm{e}^{\mathrm{i} t L_{0, n}} .
$$

The Neumann series gives the expansion

$$
U_{n}(t)=I+\mathrm{i} \int_{0}^{t} H_{n}\left(t_{1}\right) \mathrm{d} t_{1}+\sum_{\nu=2}^{\infty} \mathrm{i}^{\nu} \int_{0}^{t} \mathrm{~d} t_{1} \ldots \int_{0}^{t_{\nu-1}} H_{n}\left(t_{1}\right) \ldots H_{n}\left(t_{\nu}\right) \mathrm{d} t_{\nu} .
$$

For $\nu \geq 1$ and $\underline{t}=\left(t_{1}, \ldots, t_{\nu}\right) \in \mathbb{R}^{\nu}$ we denote

$$
H_{n}(\underline{t}):=H_{n}\left(t_{1}\right) \ldots H_{n}\left(t_{\nu}\right)
$$

and

$$
g_{\nu, n, j}(\underline{t}):=\mathrm{i}^{\nu} H_{n}(\underline{t})(j, j) .
$$

Note that $\left\langle\mathrm{e}_{j}, H_{n}(t) \mathrm{e}_{j}\right\rangle=\left\langle\mathrm{e}^{\mathrm{i} t L_{0, n}} \mathrm{e}_{j}, \tilde{V}_{n} \mathrm{e}^{\mathrm{i} t L_{0, n}} \mathrm{e}_{j}\right\rangle=\left\langle\mathrm{e}^{\mathrm{i} t l_{n}(j)} \mathrm{e}_{j}, \tilde{V}_{n} \mathrm{e}^{\mathrm{i} t l_{n}(j)} \mathrm{e}_{j}\right\rangle=\left\langle\mathrm{e}_{j}, \tilde{V}_{n} \mathrm{e}_{j}\right\rangle$, i.e.

$$
H_{n}(t)(j, j)=g_{n}(j) \text {. }
$$

For $t \in \mathbb{R}$ we denote

$$
u_{n, j}(t):=U_{n}(t)(j, j) .
$$

Then, using (4.4), we get the expansion

$$
\partial_{t} u_{n, j}(t)=\mathrm{i} g_{n}(j)+\sum_{\nu=2}^{\infty} u_{\nu, n, j}(t)
$$

where

$$
\begin{aligned}
& u_{2, n, j}(t):=-\int_{0}^{t}\left\langle\mathrm{e}_{j}, H_{n}(t) H_{n}\left(t_{2}\right) \mathrm{e}_{j}\right\rangle \mathrm{d} t_{2}, \\
& u_{\nu, n, j}(t):=\mathrm{i}^{\nu} \int_{0}^{t} \mathrm{~d} t_{2} \ldots \int_{0}^{t_{\nu-1}}\left\langle\mathrm{e}_{j}, H_{n}(t) H_{n}\left(t_{2}\right) \ldots H_{n}\left(t_{\nu}\right) \mathrm{e}_{j}\right\rangle \mathrm{d} t_{\nu} \text { for } \nu \geq 3 .
\end{aligned}
$$

Proposition 4.1. Let $t_{0}>0$ and $\varepsilon>0$.

(a) We can find $C>0$ such that

$$
\sup _{\substack{|j-n| \leq n^{\gamma} \\-t_{0} \leq t \leq t_{0}}}\left|u_{2, n, j}(t)\right| \leq C n^{-\gamma+5 \varepsilon} .
$$

(b) If $\varepsilon<1 / 8$, then we can find $\tilde{C}>0$ such that the estimate

$$
\sup _{|j-n| \leq n \gamma} \int_{-t_{0}}^{t_{0}} \mathrm{~d} t_{\nu-1}\left|\int_{0}^{t_{\nu-1}}\left\langle\mathrm{e}_{j}, H_{n}\left(t_{1}\right) \ldots H_{n}\left(t_{\nu}\right) \mathrm{e}_{j}\right\rangle \mathrm{d} t_{\nu}\right| \leq \tilde{C}^{\nu} n^{-\gamma+5 \varepsilon}
$$

holds whenever $3 \leq \nu \leq n^{\varepsilon}$ and $t_{1}, \ldots, t_{\nu-2} \in\left[-t_{0}, t_{0}\right]$.

Proof. See Section 7.

Remark. In [5, estimate (6.17b)] the constant in the right hand side should be $C^{\nu}$ instead of $C$. 
4.2. Use of the Fourier transform. In this section we prove the desired trace estimate provided assumption (4.8) is satisfied, and in the next section we show that Proposition 4.1precisely implies this assumption.

Lemma 4.2. Let $g_{n}$ be defined by (2.29) and $u_{n, j}$ by (4.5). Let $\varepsilon>0$ be fixed and assume that for every $t_{0}>0$ one has the estimate

$$
\sup _{\substack{|j-n| \leq n^{\gamma} \\-t_{0} \leq t \leq t_{0}}}\left|\partial_{t} u_{n, j}(t)-\mathrm{i} g_{n}(j)\right|=\mathrm{O}\left(n^{-\gamma+5 \varepsilon}\right) .
$$

Let $\tilde{\mathcal{G}}_{n}^{0}$ be defined by (2.36a by means of a function $\chi \in \mathcal{S}(\mathbb{R})$ whose Fourier transform has compact support. We have then the estimate

$$
\tilde{\mathcal{G}}_{n}^{0}=\mathrm{O}\left(n^{-\gamma+6 \varepsilon}\right) .
$$

Proof. It consists in four steps.

Step 1. Let $\theta_{0}$ be as in (2.1a) and $\theta_{n^{\gamma}, n}(s):=\theta_{0}\left(\frac{s-n}{n^{\gamma}}\right)$ according to (2.1b). We claim that

$$
\begin{aligned}
\left\|\left(I-\theta_{n^{\gamma}, n}\left(\tilde{L}_{0, n}\right)\right) \chi\left(L_{n}-l_{n}(n)\right)\right\|_{\mathcal{B}_{1}\left(l^{2}(\mathbb{Z})\right)} & =\mathrm{O}\left(n^{-\gamma}\right), \\
\left\|\left(I-\theta_{n^{\gamma}, n}\left(\tilde{L}_{0, n}\right)\right) \chi\left(\tilde{L}_{0, n}-l_{n}(n)\right)\right\|_{\mathcal{B}_{1}\left(l^{2}(\mathbb{Z})\right)} & =\mathrm{O}\left(n^{-\gamma}\right),
\end{aligned}
$$

where $\|T\|_{\mathcal{B}_{1}\left(l^{2}(\mathbb{Z})\right)}=\operatorname{tr} \sqrt{T^{*} T}$ is the trace class norm on the algebra $\mathcal{B}_{1}\left(l^{2}(\mathbb{Z})\right)$ of trace class operators on $l^{2}(\mathbb{Z})$. It suffices to apply [5, Proof of Lemma 6.1] with $\tilde{L}_{0, n}$ instead of $L_{0, n}$.

Step 2. The assertions (4.10) of Step 1 ensure that

$$
\tilde{\mathcal{G}}_{n}^{0}-\tilde{\mathcal{G}}_{n}=\mathrm{O}\left(n^{-\gamma}\right)
$$

holds with

$$
\tilde{\mathcal{G}}_{n}:=\operatorname{tr}\left(\theta_{n^{\gamma}, n}\left(\tilde{L}_{0, n}\right)\left(\chi\left(L_{n}-l_{n}(n)\right)-\chi\left(\tilde{L}_{0, n}-l_{n}(n)\right)\right)\right) .
$$

Thus it remains to prove $\tilde{\mathcal{G}}_{n}=\mathrm{O}\left(n^{-\gamma+6 \varepsilon}\right)$.

Step 3. Let $t_{0}>0$ be such that supp $\hat{\chi} \subset\left[-t_{0}, t_{0}\right]$. Then the inverse Fourier formula

$$
\chi(\lambda)=\int_{-\infty}^{\infty} \hat{\chi}(t) \mathrm{e}^{\mathrm{i} t \lambda} \mathrm{d} t=\int_{-t_{0}}^{t_{0}} \hat{\chi}(t) \mathrm{e}^{\mathrm{i} t \lambda} \mathrm{d} t
$$

allows us to express

$$
\chi\left(L_{n}-l_{n}(n)\right)-\chi\left(\tilde{L}_{0, n}-l_{n}(n)\right)=\int_{-t_{0}}^{t_{0}} \hat{\chi}(t) \mathrm{e}^{-\mathrm{i} t l_{n}(n)}\left(\mathrm{e}^{\mathrm{i} t L_{n}}-\mathrm{e}^{\mathrm{i} t \tilde{L}_{0, n}}\right) \mathrm{d} t
$$

and

$$
\tilde{\mathcal{G}}_{n}=\int_{-t_{0}}^{t_{0}} \hat{\chi}(t) \mathrm{e}^{-\mathrm{i} t l_{n}(n)} \operatorname{tr}\left(\theta_{n^{\gamma}, n}\left(\tilde{L}_{0, n}\right) \mathrm{e}^{\mathrm{i} t L_{0, n}}\left(U_{n}(t)-\mathrm{e}^{\mathrm{i} t g_{n}(\Lambda)}\right)\right) \mathrm{d} t .
$$

We thus have $\tilde{\mathcal{G}}_{n}=\sum_{j \in \mathbb{Z}} \tilde{\mathcal{G}}_{n}(j)$ with

$$
\tilde{\mathcal{G}}_{n}(j):=\int_{-t_{0}}^{t_{0}} \hat{\chi}(t) \mathrm{e}^{\mathrm{i} t / 2} \mathrm{e}^{\mathrm{i} t\left(l_{n}(j)-l_{n}(n)-1 / 2\right)} \theta_{n^{\gamma}, n}\left(\tilde{l}_{n}(j)\right)\left(u_{n, j}(t)-\mathrm{e}^{\mathrm{i} t g_{n}(j)}\right) \mathrm{d} t .
$$

Integrating by parts as in [5, Section 6.3$]$ we find

$$
\tilde{\mathcal{G}}_{n}(j)=\mathrm{i} \tilde{\mathcal{G}}_{1, n}(j)+\mathrm{i} \tilde{\mathcal{G}}_{2, n}(j)
$$


with

$$
\begin{aligned}
& \tilde{\mathcal{G}}_{1, n}(j)=\int_{-t_{0}}^{t_{0}} \hat{\chi}(t) \mathrm{e}^{\mathrm{i} t\left(l_{n}(j)-l_{n}(n)\right)} \frac{\theta_{n^{\gamma}, n}\left(\tilde{l}_{n}(j)\right)}{l_{n}(j)-l_{n}(n)-\frac{1}{2}} \partial_{t}\left(u_{n, j}(t)-\mathrm{e}^{\mathrm{i} t g_{n}(j)}\right) \mathrm{d} t, \\
& \tilde{\mathcal{G}}_{2, n}(j)=\int_{-t_{0}}^{t_{0}} \partial_{t}\left(\hat{\chi}(t) \mathrm{e}^{\mathrm{i} t / 2}\right) \mathrm{e}^{\mathrm{i} t\left(l_{n}(j)-l_{n}(n)-1 / 2\right)} \frac{\theta_{n^{\gamma}, n}\left(\tilde{l}_{n}(j)\right)}{l_{n}(j)-l_{n}(n)-\frac{1}{2}}\left(u_{n, j}(t)-\mathrm{e}^{\mathrm{i} t g_{n}(j)}\right) \mathrm{d} t
\end{aligned}
$$

and we can estimate

$$
\begin{aligned}
& \left|\tilde{\mathcal{G}}_{1, n}(j)\right| \leq C \frac{\theta_{n^{\gamma}, n}\left(\tilde{l}_{n}(j)\right)}{1+|j-n|} \sup _{-t_{0} \leq t \leq t_{0}}\left|\partial_{t}\left(u_{n, j}(t)-\mathrm{e}^{\mathrm{i} t g_{n}(j)}\right)\right|, \\
& \left|\tilde{\mathcal{G}}_{2, n}(j)\right| \leq C \frac{\theta_{n^{\gamma}, n}\left(\tilde{l}_{n}(j)\right)}{1+|j-n|} \sup _{-t_{0} \leq t \leq t_{0}}\left|u_{n, j}(t)-\mathrm{e}^{\mathrm{i} t g_{n}(j)}\right| .
\end{aligned}
$$

Step 4 (last step). Since $\left|\tilde{l}_{n}(j)-j\right| \leq C$ we can find $n_{0}$ such that $\theta_{n^{\gamma}, n}\left(\tilde{l}_{n}(j)\right) \neq 0$ implies $|j-n| \leq n^{\gamma}$ for $n \geq n_{0}$. Combining this fact with

$$
\sup _{-t_{0} \leq t \leq t_{0}}\left|u_{n, j}(t)-\mathrm{e}^{\mathrm{i} t g_{n}(j)}\right| \leq\left|t_{0}\right| \sup _{-t_{0} \leq t \leq t_{0}}\left|\partial_{t}\left(u_{n, j}(t)-\mathrm{e}^{\mathrm{i} t g_{n}(j)}\right)\right|
$$

we can estimate

$$
\left|\tilde{\mathcal{G}}_{n}\right| \leq \sum_{|j-n| \leq n \gamma} \frac{C_{0}}{1+|j-n|} \sup _{-t_{0} \leq t \leq t_{0}}\left|\partial_{t}\left(u_{n, j}(t)-\mathrm{e}^{\mathrm{i} t g_{n}(j)}\right)\right| .
$$

By (2.46) we have the estimate $\left|\mathrm{e}^{\mathrm{i} t g_{n}(j)}-1\right| \leq\left|t g_{n}(j)\right| \leq C|t| n^{-\gamma / 2}$ for $|j-n| \leq n^{\gamma}$, and thus

$$
\sup _{\substack{|j-n| \leq n^{\gamma} \\-t_{0} \leq t \leq t_{0}}}\left|\partial_{t} \mathrm{e}^{\mathrm{i} t g_{n}(j)}-\mathrm{i} g_{n}(j)\right|=\mathrm{O}\left(n^{-\gamma}\right)
$$

Therefore combining assumption (4.8) with (4.13) we find

$$
\sup _{\substack{|j-n| \leq n^{\gamma} \\-t_{0} \leq t \leq t_{0}}}\left|\partial_{t}\left(u_{n, j}(t)-\mathrm{e}^{\mathrm{i} t g_{n}(j)}\right)\right|=\mathrm{O}\left(n^{-\gamma+5 \varepsilon}\right) .
$$

Using

$$
\sum_{|k| \leq n^{\gamma}} \frac{1}{1+|k|} \leq 1+2 \ln (n)=\mathrm{O}\left(n^{\varepsilon}\right)
$$

and (4.14) we can estimate the r.h.s. of (4.12) by $\mathrm{O}\left(n^{-\gamma+6 \varepsilon}\right)$, hence (4.9) follows from (4.11).

4.3. Proof of Proposition $4.1 \Longrightarrow$ Proposition 2.4. Since $\varepsilon>0$ is arbitrary, it is clear that it suffices to prove $\tilde{\mathcal{G}}_{n}^{0}=\mathrm{O}\left(n^{-\gamma+6 \varepsilon}\right)$ instead of (2.39). Thus by Lemma 4.2 it only remains to check that the assertions of Proposition 4.1 imply estimate (4.8), i.e.,

$$
\sup _{\substack{|j-n| \leq n^{\gamma} \\-t_{0} \leq t \leq t_{0}}}\left|\partial_{t} u_{n, j}(t)-\mathrm{i} g_{n}(j)\right|=\mathrm{O}\left(n^{-\gamma+5 \varepsilon}\right)
$$

We first note that (4.6) and (4.4) give the expansion

$$
\partial_{t} u_{n, j}(t)-\mathrm{i} g_{n}(j)=\sum_{\nu=2}^{\infty} u_{\nu, n, j}(t) .
$$

We then observe that (4.7b) for $\nu=3$ yields

$$
\sup _{\substack{|j-n| \leq n^{\gamma} \\-t_{0} \leq t \leq t_{0}}}\left|u_{3, n, j}(t)\right| \leq \tilde{C}^{3} n^{-\gamma+5 \varepsilon} .
$$


For any $4 \leq \nu<n^{\varepsilon}$ and $t \in\left[-t_{0}, t_{0}\right]$, estimate (4.7b) gives

$$
\sup _{|j-n| \leq n \gamma}\left|u_{\nu, n, j}(t)\right| \leq \tilde{C}^{\nu} n^{-\gamma+5 \varepsilon} \int_{\Delta_{t}} \mathrm{~d} t_{2} \cdots \int_{\Delta_{t_{\nu-3}}} \mathrm{~d} t_{\nu-2}=\tilde{C}^{\nu} n^{-\gamma+5 \varepsilon} \frac{|t|^{\nu-3}}{(\nu-3) !},
$$

where $\Delta_{t}:=[0, t]$ when $t \geq 0$ and $[t, 0]$ when $t \leq 0$. Therefore, by using (4.7a), (4.16), and (4.17) we get

$$
\sup _{\substack{|j-n| \leq n^{\gamma} \\-t_{0} \leq t \leq t_{0}}} \sum_{\substack{2 \leq \nu<n^{\varepsilon} \\ \mid u_{\nu, n, j}}}\left|u_{i}\right| \leq C n^{-\gamma+5 \varepsilon}+\sum_{3 \leq \nu<n^{\varepsilon}} \frac{\tilde{C}^{\nu} t_{0}^{\nu-3} n^{-\gamma+5 \varepsilon}}{(\nu-3) !} \leq\left(C+\tilde{C}^{3} \mathrm{e}^{\tilde{C} t_{0}}\right) n^{-\gamma+5 \varepsilon} .
$$

To complete the proof it remains to consider indices $\nu \geq n^{\varepsilon}$. We observe that $\left\|H_{n}(t)\right\|=\left\|\tilde{V}_{n}\right\|=$ $\left\|V_{n}\right\| \leq \rho_{N}$. Therefore,

$$
\left|\left\langle\mathrm{e}_{j}, H_{n}(t) H_{n}\left(t_{2}\right) \ldots H_{n}\left(t_{\nu}\right) \mathrm{e}_{j}\right\rangle\right| \leq \rho_{N}^{\nu}
$$

and

$$
\left|u_{\nu, n, j}(t)\right| \leq \int_{\Delta_{t}} \mathrm{~d} t_{2} \cdots \int_{\Delta_{t_{\nu-1}}}\left|\left\langle\mathrm{e}_{j}, H_{n}(t) H_{n}\left(t_{2}\right) \ldots H_{n}\left(t_{\nu}\right) \mathrm{e}_{j}\right\rangle\right| \mathrm{d} t_{\nu} \leq \rho_{N}^{\nu} \frac{|t|^{\nu-1}}{(\nu-1) !} .
$$

We thus get

$$
\sup _{\substack{|j-n| \leq n^{\gamma} \\-t_{0} \leq t \leq t_{0}}} \sum_{\nu \geq n^{\varepsilon}}\left|u_{\nu, n, j}(t)\right| \leq \sum_{\nu \geq n^{\varepsilon}} \frac{\rho_{N}^{\nu} t_{0}^{\nu-1}}{(\nu-1) !} \leq \frac{\rho_{N} \mathrm{e}^{\rho_{N} t_{0}}\left(\rho_{N} t_{0}\right)^{\left\lfloor n^{\varepsilon}\right\rfloor-1}}{\left(\left\lfloor n^{\varepsilon}\right\rfloor-1\right) !}=\mathrm{O}\left(n^{-m}\right)
$$

for any integer $m$. Estimates (4.18) with (4.15) show that assumption (4.8) in Lemma 4.2 is valid.

\section{Estimate OF SOME OSCILLATORY INTEGRALS}

5.1. Main result. In this section we consider oscillatory integrals of the following type:

$$
\mathcal{J}\left(b, t_{1}, t_{2}, \zeta, \mu\right):=\int_{t_{1}}^{t_{2}} \frac{\mathrm{e}^{\mathrm{i} \mu \sqrt{4 \sin ^{2}(t / 2)+\zeta^{2}}}}{\left(4 \sin ^{2}(t / 2)+\zeta^{2}\right)^{1 / 4}} b(t) \mathrm{d} t,
$$

where $b \in \mathrm{C}^{1}(\mathbb{R}), \mu \in \mathbb{R}^{*}, \zeta \geq 0$ and $t_{1} \leq t_{2}$ are real numbers. Notice that in the case $\zeta=0$ the function $t \rightarrow|2 \sin (t / 2)|^{-1 / 2}$ is Lebesgue integrable on any bounded interval of $\mathbb{R}$ and so (5.1) is still well defined.

Lemma 5.1. Let $\Delta_{0} \subset \mathbb{R}$ be a bounded interval. Then there is a constant $C_{\Delta_{0}}>0$ such that for any interval $\left[t_{1}, t_{2}\right] \subset \Delta_{0}, \mu \in \mathbb{R}^{*}, \zeta \geq 0$ and $b \in \mathrm{C}^{1}(\mathbb{R})$ one has the estimate

$$
\left|\mathcal{J}\left(b, t_{1}, t_{2}, \zeta, \mu\right)\right| \leq C_{\Delta_{0}} \frac{1+\sqrt{\zeta}}{\sqrt{|\mu|}} \mathcal{M}\left(b,\left[t_{1}, t_{2}\right]\right),
$$

where for any bounded interval $\Delta \subset \mathbb{R}$

$$
\mathcal{M}(b, \Delta):=\sup _{t \in \Delta}|b(t)|+\int_{\Delta}\left|b^{\prime}(t)\right| \mathrm{d} t .
$$

This lemma is used in the proof of Proposition 4.1. It serves in Section 7.3.4 to prove estimate (7.19) of some oscillatory integral.

Proof. The proof is given in the next subsections. It is based on van der Corput lemma. 
Lemma 5.2 (van der Corput). Assume that $h_{0}:\left(t_{1}, t_{2}\right) \rightarrow \mathbb{R}$ is smooth and its second derivative satisfies $h_{0}^{\prime \prime}(t) \geq c_{0}$ for $t_{1}<t<t_{2}$ and some constant $c_{0}>0$. Assume also that $\mu_{0} \in \mathbb{R}^{*}$ and $b_{0} \in \mathrm{C}^{1}\left(\left(t_{1}, t_{2}\right)\right)$, and consider the oscillatory integral

$$
\mathcal{J}\left(b_{0}, t_{1}, t_{2}, h_{0}, \mu_{0}\right):=\int_{t_{1}}^{t_{2}} \mathrm{e}^{\mathrm{i} \mu_{0} h_{0}(t)} b_{0}(t) \mathrm{d} t .
$$

Then there is a constant $C_{0}$ depending only on $c_{0}$ such that we have the estimate

$$
\left|\mathcal{J}\left(b_{0}, t_{1}, t_{2}, h_{0}, \mu_{0}\right)\right| \leq \frac{C_{0}}{\sqrt{\left|\mu_{0}\right|}}\left(\left|b_{0}\left(t_{1}\right)\right|+\int_{t_{1}}^{t_{2}}\left|b_{0}^{\prime}(t)\right| \mathrm{d} t\right) \leq \frac{C_{0}}{\sqrt{\left|\mu_{0}\right|}} \mathcal{M}\left(b_{0},\left[t_{1}, t_{2}\right]\right) .
$$

Proof. See [18, Section VIII.1.2, p. 354].

\subsection{Proof of Lemma 5.1 in case $\Delta_{0}=[0,2 \pi / 3]$.}

5.2.1. Change of variable. By our assumption $\left[t_{1}, t_{2}\right] \subset[0,2 \pi / 3]$ and the change of variable

$$
t=2 \arcsin (s / 2)
$$

parametrize $[0,2 \pi / 3]$ by $s \in[0, \sqrt{3}]$. If $t \in\left[t_{1}, t_{2}\right]$ then $s \in\left[s_{1}, s_{2}\right]$ where $s_{i}:=2 \sin \left(t_{i} / 2\right), i=1,2$. In particular, $\sqrt{4 \sin ^{2}(t / 2)+\zeta^{2}}=\sqrt{s^{2}+\zeta^{2}}$ and the change of variable (5.5) gives

$$
\mathcal{J}\left(b, t_{1}, t_{2}, \zeta, \mu\right)=\mathcal{J}_{1}\left(b_{1}, s_{1}, s_{2}, \zeta, \mu\right),
$$

where

with

$$
\mathcal{J}_{1}\left(b_{1}, s_{1}, s_{2}, \zeta, \mu\right):=\int_{s_{1}}^{s_{2}} \frac{\mathrm{e}^{\mathrm{i} \mu \sqrt{s^{2}+\zeta^{2}}}}{\left(s^{2}+\zeta^{2}\right)^{1 / 4}} b_{1}(s) \mathrm{d} s
$$

$$
b_{1}(s):=\frac{b(2 \arcsin (s / 2))}{\sqrt{1-s^{2} / 4}} .
$$

Since there is a constant $C_{0}$ such that $\mathcal{M}\left(b_{1},\left[s_{1}, s_{2}\right]\right) \leq C_{0} \mathcal{M}\left(b,\left[t_{1}, t_{2}\right]\right)$, to get (5.2) for any interval $\left[t_{1}, t_{2}\right] \subset[0,2 \pi / 3], \mu \in \mathbb{R}^{*}, \zeta \geq 0$, and $b \in \mathrm{C}^{1}\left(\left[t_{1}, t_{2}\right]\right)$ it suffices to prove the following

Statement. There is a constant $C>0$ such that for any $\mu \in \mathbb{R}^{*}, \zeta \geq 0,\left[s_{1}, s_{2}\right] \subset[0, \sqrt{3}]$, and $b_{1} \in \mathrm{C}^{1}\left(\left[s_{1}, s_{2}\right]\right)$ we have the estimate

$$
\left|\mathcal{J}_{1}\left(b_{1}, s_{1}, s_{2}, \zeta, \mu\right)\right| \leq \frac{C}{\sqrt{|\mu|}} \mathcal{M}\left(b_{1},\left[s_{1}, s_{2}\right]\right) .
$$

To prove this statement we distinguish three cases: $\zeta \geq s_{2}, \zeta \leq s_{1}$, and $s_{1} \leq \zeta \leq s_{2}$.

5.2.2. Proof of (5.6) in case $\zeta \geq s_{2}$. Using the notations

$$
h_{1}(s, \zeta):=\zeta \sqrt{\zeta^{2}+s^{2}}
$$

and (5.4) from Lemma 5.2 we can write

$$
\begin{aligned}
\mathcal{J}_{1}\left(b_{1}, s_{1}, s_{2}, \zeta, \mu\right) & =\sqrt{\zeta} \int_{s_{1}}^{s_{2}} \mathrm{e}^{\mathrm{i} \mu \zeta^{-1} h_{1}(s, \zeta)} h_{1}(s, \zeta)^{-1 / 2} b_{1}(s) \mathrm{d} s \\
& =\sqrt{\zeta} \times \mathcal{J}\left(b_{1} \tilde{h}_{1}(\cdot, \zeta), s_{1}, s_{2}, h_{1}(\cdot, \zeta), \mu \zeta^{-1}\right)
\end{aligned}
$$

where

$$
\tilde{h}_{1}(s, \zeta):=h_{1}(s, \zeta)^{-1 / 2}=\frac{1}{\sqrt{\zeta}\left(\zeta^{2}+s^{2}\right)^{1 / 4}} .
$$

Next we observe that $\zeta \geq s$ ensures

$$
\partial_{s}^{2} h_{1}(s, \zeta)=\frac{\zeta^{3}}{\left(s^{2}+\zeta^{2}\right)^{3 / 2}} \geq \frac{\zeta^{3}}{\left(2 \zeta^{2}\right)^{3 / 2}}=2^{-3 / 2}
$$


and thus we can apply Lemma 5.2 It gives the estimate

$$
\left|\mathcal{J}_{1}\left(b_{1}, s_{1}, s_{2}, \zeta, \mu\right)\right| \leq \frac{C_{0} \zeta}{\sqrt{|\mu|}} \mathcal{M}\left(b_{1} \tilde{h}_{1}(\cdot, \zeta),\left[s_{1}, s_{2}\right]\right) .
$$

Thus to get (5.6) it suffices to show

$$
\mathcal{M}\left(b_{1} \tilde{h}_{1}(\cdot, \zeta),\left[s_{1}, s_{2}\right]\right) \leq 2 \frac{\mathcal{M}\left(b_{1},\left[s_{1}, s_{2}\right]\right)}{\zeta} .
$$

For this purpose we first observe that $h_{1}(s, \zeta):=\zeta \sqrt{\zeta^{2}+s^{2}} \geq \zeta^{2}$. Then

$$
0<\tilde{h}_{1}(s, \zeta) \leq \frac{1}{\zeta},
$$

hence

$$
\sup _{s_{1} \leq s \leq s_{2}}\left|b_{1}(s) \tilde{h}_{1}(s, \zeta)\right| \leq \sup _{s_{1} \leq s \leq s_{2}} \frac{\left|b_{1}(s)\right|}{\zeta} \leq \frac{\mathcal{M}\left(b_{1},\left[s_{1}, s_{2}\right]\right)}{\zeta} .
$$

Next we claim that

$$
\int_{s_{1}}^{s_{2}}\left|\partial_{s} \tilde{h}_{1}(s, \zeta)\right| \mathrm{d} s \leq \frac{1}{\zeta} .
$$

Indeed, since $\partial_{s} \tilde{h}_{1}(s, \zeta) \leq 0$, we can estimate the left hand side of (5.8) using (5.7):

$$
\int_{s_{1}}^{s_{2}}\left(-\partial_{s} \tilde{h}_{1}\right)(s, \zeta) \mathrm{d} s=\tilde{h}_{1}\left(s_{1}, \zeta\right)-\tilde{h}_{1}\left(s_{2}, \zeta\right) \leq \frac{1}{\zeta} .
$$

Finally it remains to show

$$
\int_{s_{1}}^{s_{2}}\left|\partial_{s}\left(b_{1} \tilde{h}_{1}\right)(s, \zeta)\right| \mathrm{d} s \leq \frac{\mathcal{M}\left(b_{1},\left[s_{1}, s_{2}\right]\right)}{\zeta} .
$$

The left hand side of (5.9) is indeed $\int_{s_{1}}^{s_{2}}\left|b_{1}^{\prime}(s) \tilde{h}_{1}(s, \zeta)+b_{1}(s) \partial_{s} \tilde{h}_{1}(s, \zeta)\right| \mathrm{d} s$. Thus, using (5.7) and (5.8) we get

$$
\begin{aligned}
\int_{s_{1}}^{s_{2}}\left|\partial_{s}\left(b_{1} \tilde{h}_{1}\right)(s, \zeta)\right| \mathrm{d} s & \leq \sup _{s_{1} \leq s \leq s_{2}}\left|\tilde{h}_{1}(s, \zeta)\right| \int_{s_{1}}^{s_{2}}\left|b_{1}^{\prime}(s)\right| \mathrm{d} s+\sup _{s_{1} \leq s \leq s_{2}}\left|b_{1}(s)\right| \int_{s_{1}}^{s_{2}}\left|\partial_{s} \tilde{h}_{1}(s, \zeta)\right| \mathrm{d} s \\
& \leq \frac{1}{\zeta}\left(\sup _{s_{1} \leq s \leq s_{2}}\left|b_{1}(s)\right|+\int_{s_{1}}^{s_{2}}\left|b_{1}^{\prime}(s)\right| \mathrm{d} s\right)=\frac{\mathcal{M}\left(b_{1},\left[s_{1}, s_{2}\right]\right)}{\zeta} .
\end{aligned}
$$

5.2.3. Proof of (5.6) in case $\zeta \leq s_{1}$. We denote $\tilde{s}_{i}:=\left(s_{i}^{2}+\zeta^{2}\right)^{1 / 4}, i=1,2$ and consider the change of variable

$$
s=\sqrt{\tilde{s}^{4}-\zeta^{2}} \text { for } \tilde{s} \in\left[\tilde{s}_{1}, \tilde{s}_{2}\right]
$$

which gives $\sqrt{s^{2}+\zeta^{2}}=\tilde{s}^{2}$. By applying this change of variable to the integral

$$
\mathcal{J}_{1}\left(b_{1}, s_{1}, s_{2}, \zeta, \mu\right)=\int_{s_{1}}^{s_{2}} \mathrm{e}^{\mathrm{i} \mu \sqrt{s^{2}+\zeta^{2}}}\left(s^{2}+\zeta^{2}\right)^{-1 / 4} b_{1}(s) \mathrm{d} s
$$

we find

$$
\mathcal{J}_{1}\left(b_{1}, s_{1}, s_{2}, \zeta, \mu\right)=\int_{\tilde{s}_{1}}^{\tilde{s}_{2}} \mathrm{e}^{\mathrm{i} \mu \tilde{s}^{2}} b_{2}(\tilde{s}, \zeta) \tilde{h}_{2}(\tilde{s}, \zeta) \mathrm{d} \tilde{s}=\mathcal{J}\left(b_{2} \tilde{h}_{2}(\cdot, \zeta), \tilde{s}_{1}, \tilde{s}_{2}, h_{0}, \mu\right),
$$

with $b_{2}(\tilde{s}, \zeta):=b_{1}\left(\sqrt{\tilde{s}^{4}-\zeta^{2}}\right), h_{0}(\tilde{s})=\tilde{s}^{2}$ and

$$
\tilde{h}_{2}(\tilde{s}, \zeta):=\frac{1}{\tilde{s}} \partial_{\tilde{s}}\left(\sqrt{\tilde{s}^{4}-\zeta^{2}}\right)=\frac{2 \tilde{s}^{2}}{\sqrt{\tilde{s}^{4}-\zeta^{2}}} .
$$


Then Lemma 5.2 applies and gives

$$
\left|\mathcal{J}_{1}\left(b_{1}, s_{1}, s_{2}, \zeta, \mu\right)\right| \leq \frac{C_{0}}{\sqrt{|\mu|}} \mathcal{M}\left(b_{2} \tilde{h}_{2},\left[\tilde{s}_{1}, \tilde{s}_{2}\right]\right) .
$$

We observe that $s \geq s_{1} \geq \zeta$ ensures $\tilde{s} \geq\left(2 \zeta^{2}\right)^{1 / 4}$, hence $\zeta \leq \tilde{s}^{2} / \sqrt{2}$. Using the fact that $\tilde{h}_{2}(s, \zeta)$ is increasing with $\zeta$ we have the estimate

$$
s \geq s_{1} \geq \zeta \Longrightarrow \tilde{h}_{2}(\tilde{s}, \zeta) \leq \tilde{h}_{2}\left(\tilde{s}, \tilde{s}^{2} / \sqrt{2}\right)=2 \sqrt{2} .
$$

Moreover, $\partial_{\tilde{s}} \tilde{h}_{2}(\tilde{s}, \zeta)=-4 \zeta^{2} \tilde{s}\left(\tilde{s}^{4}-\zeta^{2}\right)^{-3 / 2} \leq 0$ and as in the proof of estimate (5.8) we find

$$
\int_{\tilde{s}_{1}}^{\tilde{s}_{2}}\left|\partial_{\tilde{s}} \tilde{h}_{2}(\tilde{s}, \zeta)\right| \mathrm{d} \tilde{s}=\tilde{h}_{2}\left(\tilde{s}_{1}, \zeta\right)-\tilde{h}_{2}\left(\tilde{s}_{2}, \zeta\right) \leq 2 \sqrt{2}
$$

Estimate (5.10) leads to the desired estimate (5.6) if we prove

$$
\mathcal{M}\left(b_{2} \tilde{h}_{2},\left[\tilde{s}_{1}, \tilde{s}_{2}\right]\right) \leq 4 \sqrt{2} \mathcal{M}\left(b_{2},\left[\tilde{s}_{1}, \tilde{s}_{2}\right]\right)=4 \sqrt{2} \mathcal{M}\left(b_{1},\left[s_{1}, s_{2}\right]\right) .
$$

The last equality is easy. For the inequality we first observe that by (5.11)

$$
\sup _{\tilde{s}_{1} \leq \tilde{s} \leq \tilde{s}_{2}}\left|b_{2} \tilde{h}_{2}(\tilde{s}, \zeta)\right| \leq 2 \sqrt{2} \sup _{\tilde{s}_{1} \leq \tilde{s} \leq \tilde{s}_{2}}\left|b_{2}(\tilde{s}, \zeta)\right| \leq 2 \sqrt{2} \mathcal{M}\left(b_{2},\left[\tilde{s}_{1}, \tilde{s}_{2}\right]\right) .
$$

Moreover, using (5.11) and (5.12) we find

$$
\begin{aligned}
\int_{\tilde{s}_{1}}^{\tilde{s}_{2}}\left|\partial_{\tilde{s}}\left(b_{2} \tilde{h}_{2}\right)(\tilde{s}, \zeta)\right| \mathrm{d} \tilde{s} & \leq \sup _{\tilde{s}_{1} \leq \tilde{s} \leq \tilde{s}_{2}}\left|\tilde{h}_{2}(\tilde{s}, \zeta)\right| \int_{\tilde{s}_{1}}^{\tilde{s}_{2}}\left|\partial_{\tilde{s}} b_{2}(\tilde{s}, \zeta)\right| \mathrm{d} \tilde{s}+\sup _{\tilde{s}_{1}<\tilde{s}<\tilde{s}_{2}}\left|b_{2}(\tilde{s}, \zeta)\right| \int_{\tilde{s}_{1}}^{\tilde{s}_{2}}\left|\partial_{\tilde{s}} \tilde{h}_{2}(\tilde{s}, \zeta)\right| \mathrm{d} \tilde{s} \\
& \leq 2 \sqrt{2} \int_{\tilde{s}_{1}}^{\tilde{s}_{2}}\left|\partial_{\tilde{s}} b_{2}(\tilde{s}, \zeta)\right| \mathrm{d} \tilde{s}+2 \sqrt{2} \sup _{\tilde{s}_{1}<\tilde{s}<\tilde{s}_{2}}\left|b_{2}(\tilde{s}, \zeta)\right| \\
& =2 \sqrt{2} \mathcal{M}\left(b_{2},\left[\tilde{s}_{1}, \tilde{s}_{2}\right]\right) .
\end{aligned}
$$

5.2.4. Proof of (5.6) in case $s_{1}<\zeta<s_{2}$. This case reduces to the previous ones. For $s_{1}<\zeta<s_{2}$ we indeed have

$$
\begin{aligned}
& \mathcal{J}_{1}\left(b_{1}, s_{1}, s_{2}, \zeta, \mu\right)=\mathcal{J}_{1}\left(b_{1}, s_{1}, \zeta, \zeta, \mu\right)+\mathcal{J}_{1}\left(b_{1}, \zeta, s_{2}, \zeta, \mu\right), \\
& \mathcal{M}\left(b_{1},\left[s_{1}, \zeta\right]\right)+\mathcal{M}\left(b_{1},\left[\zeta, s_{2}\right]\right) \leq 2 \mathcal{M}\left(b_{1},\left[s_{1}, s_{2}\right]\right) .
\end{aligned}
$$

5.3. Proof of Lemma 5.1 in case $\Delta_{0}=[2 \pi / 3, \pi]$. Denoting

$$
h(t, \zeta):=\sqrt{4 \sin ^{2}(t / 2)+\zeta^{2}}
$$

we can write

$$
\begin{aligned}
& \mathcal{J}_{1}\left(b, t_{1}, t_{2}, \zeta, \mu\right):=\int_{t_{1}}^{t_{2}} \frac{\mathrm{e}^{\mathrm{i} \mu} \sqrt{4 \sin ^{2}(t / 2)+\zeta^{2}}}{\left(4 \sin ^{2}(t / 2)+\zeta^{2}\right)^{1 / 4}} b(t) \mathrm{d} t=\int_{t_{1}}^{t_{2}} \mathrm{e}^{\mathrm{i} \mu h(t, \zeta)} \frac{b(t)}{h(t, \zeta)^{1 / 2}} \mathrm{~d} t \\
& =\int_{t_{1}}^{t_{2}} \mathrm{e}^{\mathrm{i} \frac{\mu}{1+\zeta}(1+\zeta) h(t, \zeta)} \tilde{b}(t, \zeta) \mathrm{d} t=\mathcal{J}\left(\tilde{b}, t_{1}, t_{2}, \tilde{h}, \tilde{\mu}\right),
\end{aligned}
$$

where $\tilde{b}(t, \zeta):=b(t) h(t, \zeta)^{-1 / 2}, \tilde{\mu}:=\mu /(1+\zeta), \tilde{h}(t, \zeta):=(1+\zeta) h(t, \zeta)$, and $\mathcal{J}$ is as in (5.4). We get

$$
\partial_{t} h(t, \zeta)=\frac{\sin t}{h(t, \zeta)}, \quad-\partial_{t}^{2} h(t, \zeta)=-\frac{\cos t}{h(t, \zeta)}+\frac{\sin ^{2} t}{h(t, \zeta)^{3}} .
$$

Since $2 \pi / 3 \leq t \leq \pi$ implies $1 / 2 \leq-\cos t \leq 1$, we get

$$
2 \pi / 3 \leq t \leq \pi \Longrightarrow-\partial_{t}^{2} \tilde{h}(t, \zeta) \geq-\frac{(1+\zeta) \cos t}{h(t, \zeta)} \geq \frac{1+\zeta}{2 \sqrt{4+\zeta^{2}}} \geq \frac{1}{4}
$$


Hence, Lemma 5.2 applies to estimate $\mathcal{J}\left(\tilde{b}, t_{1}, t_{2}, \tilde{h}, \tilde{\mu}\right)$. To get (5.2) it suffices to show that there exists a constant $\tilde{C}$, independent of $\zeta, t_{1}, t_{2}$ such that

$$
\mathcal{M}\left(\tilde{b},\left[t_{1}, t_{2}\right]\right) \leq \tilde{C} \mathcal{M}\left(b,\left[t_{1}, t_{2}\right]\right) .
$$

For $2 \pi / 3 \leq t \leq \pi$ we have $2 \sin (t / 2) \geq \sqrt{3}$ so that $h(t, \zeta) \geq \sqrt{3+\zeta^{2}} \geq \sqrt{3}$. Hence,

$$
\sup _{t_{1} \leq t \leq t_{2}}|\tilde{b}(t, \zeta)|=\sup _{t_{1} \leq t \leq t_{2}} \frac{|b(t)|}{\sqrt{h(t, \zeta)}} \leq \frac{1}{\sqrt[4]{3}} \sup _{t_{1} \leq t \leq t_{2}}|b(t)| .
$$

Moreover,

$$
\left|\partial_{t} \tilde{b}(t, \zeta)\right|=\left|\frac{b^{\prime}(t)}{h(t, \zeta)^{1 / 2}}-\frac{b(t) \sin t}{2 h(t, \zeta)^{5 / 2}}\right| \leq \frac{1}{\sqrt[4]{3}}\left|b^{\prime}(t)\right|+\frac{1}{2 \sqrt[4]{3^{5}}}|b(t)| \leq \frac{1}{\sqrt[4]{3}}\left(\left|b^{\prime}(t)\right|+|b(t)|\right) .
$$

Thus, using $t_{2}-t_{1} \leq \pi / 3$,

$$
\int_{t_{1}}^{t_{2}}\left|\partial_{t} \tilde{b}(t, \zeta)\right| \mathrm{d} t \leq \frac{1}{\sqrt[4]{3}} \int_{t_{1}}^{t_{2}}\left|b^{\prime}(t)\right| \mathrm{d} t+\frac{\pi}{3 \sqrt[4]{3}} \sup _{t_{1} \leq t \leq t_{2}}|b(t)| .
$$

Summarizing,

$$
\mathcal{M}\left(\tilde{b},\left[t_{1}, t_{2}\right]\right) \leq \frac{1}{\sqrt[4]{3}}\left(1+\frac{\pi}{3}\right) \mathcal{M}\left(b,\left[t_{1}, t_{2}\right]\right)
$$

\subsection{Proof of Lemma 5.1; last steps.}

5.4.1. Proof of (5.2) in case $\Delta_{0}=[0, \pi]$. By Sections 5.2 and 5.3 we know that (5.2) holds if $2 \pi / 3 \notin\left(t_{1}, t_{2}\right)$. The remaining case $t_{1}<2 \pi / 3<t_{2}$ can be deduced from the previous ones by using the additivity properties

$$
\begin{aligned}
& \mathcal{J}\left(b, t_{1}, t_{2}, \zeta, \mu\right)=\mathcal{J}\left(b, t_{1}, t_{*}, \zeta, \mu\right)+\mathcal{J}\left(b, t_{*}, t_{2}, \zeta, \mu\right), \\
& \mathcal{M}\left(b,\left[t_{1}, t_{*}\right]\right)+\mathcal{M}\left(b,\left[t_{*}, t_{2}\right]\right) \leq 2 \mathcal{M}\left(b,\left[t_{1}, t_{2}\right]\right)
\end{aligned}
$$

with $t_{*}=2 \pi / 3$.

5.4.2. Proof of (5.2) in case $\Delta_{0}=[-\pi, 0]$. This case reduces to the previous one by using the symmetry $t \rightsquigarrow-t$. We indeed have $\mathcal{J}\left(b, t_{1}, t_{2}, \zeta, \mu\right)=\mathcal{J}\left(\breve{b},-t_{2},-t_{1}, \zeta, \mu\right)$ and $\mathcal{M}\left(b,\left[t_{1}, t_{2}\right]\right)=$ $\mathcal{M}\left(\check{b},\left[-t_{2},-t_{1}\right]\right)$ where $\check{b}(t)=b(-t)$.

5.4.3. Proof of (5.2) in case $\Delta_{0}=[-\pi, \pi]$. By Sections 5.4.1 and 5.4.2 we know that (5.2) holds if $0 \notin\left(t_{1}, t_{2}\right)$. The remaining case $0 \in\left(t_{1}, t_{2}\right)$ can be deduced from the two previous ones by using (5.13) with $t_{*}=0$.

5.4.4. Proof of (5.2) for $\Delta_{0}=[(2 k-1) \pi,(2 k+1) \pi], k \in \mathbb{Z}$. This case reduces to the previous one by translation $t \mapsto \hat{t}:=t-2 k \pi$. We indeed have $\mathcal{J}\left(b, t_{1}, t_{2}, \zeta, \mu\right)=\mathcal{J}\left(\hat{b}, \hat{t}_{1}, \hat{t}_{2}, \zeta, \mu\right)$ and $\mathcal{M}\left(b,\left[t_{1}, t_{2}\right]\right)=\mathcal{M}\left(\hat{b},\left[\hat{t}_{1}, \hat{t}_{2}\right]\right)$ where $\hat{b}(t):=b(t+2 k \pi)$.

5.4.5. Proof of (5.2) for arbitrary $\Delta_{0}$. We know (5.2) holds if $\left(t_{1}, t_{2}\right) \cap(2 \mathbb{Z}+1) \pi=\emptyset$. If $\left(t_{1}, t_{2}\right) \cap(2 \mathbb{Z}+1) \pi=\left\{t_{*}^{1}, t_{*}^{2}, \ldots, t_{*}^{k}\right\}$ where $t_{*}^{2}=t_{*}^{1}+2 \pi, \ldots, t_{*}^{k}=t_{*}^{1}+2(k-1) \pi, k \in \mathbb{Z}$ then (5.2) follows by using repeatedly properties (5.13):

$$
\begin{aligned}
& \mathcal{J}\left(b, t_{1}, t_{2}, \zeta, \mu\right)=\mathcal{J}\left(b, t_{1}, t_{*}^{1}, \zeta, \mu\right)+\mathcal{J}\left(b, t_{*}^{1}, t_{*}^{2}, \zeta, \mu\right)+\cdots+\mathcal{J}\left(b, t_{*}^{k}, t_{2}, \zeta, \mu\right), \\
& \mathcal{M}\left(b,\left[t_{1}, t_{*}^{1}\right]\right)+\mathcal{M}\left(b,\left[t_{*}^{1}, t_{*}^{2}\right]\right)+\cdots+\mathcal{M}\left(b,\left[t_{*}^{k}, t_{2}\right]\right) \leq(k+1) \mathcal{M}\left(b,\left[t_{1}, t_{2}\right]\right) .
\end{aligned}
$$




\section{Approximation By OSCILLATORY INTEGRALS}

6.1. Decomposition of $\boldsymbol{H}_{\boldsymbol{n}}(\underline{\boldsymbol{t}})$ into components $\boldsymbol{H}_{\underline{\boldsymbol{\omega}}}, \underline{\underline{t}}$. We assume $\alpha_{0}=0$ in (1.12) and denote $\Omega^{*}=\{2 \pi m / N\}_{m=1}^{N-1}$. Thus, we can expand

$$
v(\Lambda)=\sum_{\omega \in \Omega^{*}} c_{\omega} \mathrm{e}^{\mathrm{i} \omega \Lambda}
$$

where $c_{\omega} \in \mathbb{C}$ are constants. Since $H_{n}(t):=\mathrm{e}^{-\mathrm{i} t L_{0, n}} \tilde{V}_{n} \mathrm{e}^{\mathrm{i} t L_{0, n}}$ with $\tilde{V}_{n}=\mathrm{e}^{\mathrm{i} B_{n}}\left(\theta_{n, n}^{2} v\right)(\Lambda) \mathrm{e}^{-\mathrm{i} B_{n}}$ we can expand $H_{n}(t)$ as follows:

$$
H_{n}(t)=\mathrm{e}^{-\mathrm{i} t L_{0, n}} \mathrm{e}^{\mathrm{i} B_{n}}\left(\theta_{n, n}^{2} v\right)(\Lambda) \mathrm{e}^{-\mathrm{i} B_{n}} \mathrm{e}^{\mathrm{i} t L_{0, n}}=\sum_{\omega \in \Omega^{*}} c_{\omega} H_{n}^{\omega, t}
$$

with

$$
H_{n}^{\omega, t}:=\mathrm{e}^{-\mathrm{i} t L_{0, n}} \mathrm{e}^{\mathrm{i} B_{n}} \theta_{n, n}^{2}(\Lambda) \mathrm{e}^{\mathrm{i} \omega \Lambda} \mathrm{e}^{-\mathrm{i} B_{n}} \mathrm{e}^{\mathrm{i} t L_{0, n}} .
$$

More generally, for any integer $\nu \geq 1, \underline{\omega}=\left(\omega_{1}, \ldots, \omega_{\nu}\right) \in\left(\Omega^{*}\right)^{\nu}$, and $\underline{t}=\left(t_{1}, \ldots, t_{\nu}\right) \in \mathbb{R}^{\nu}$,

$$
H_{n}(\underline{t}):=H_{n}\left(t_{1}\right) \ldots H_{n}\left(t_{\nu}\right)=\sum_{\underline{\omega} \in\left(\Omega^{*}\right)^{\nu}} c_{\underline{\omega}} H \frac{\omega}{n}, \underline{t}
$$

where $c_{\underline{\omega}}:=c_{\omega_{1}} \ldots c_{\omega_{\nu}}$ and

$$
H \frac{\omega}{n}, \underline{t}:=H_{n}^{\omega_{1}, t_{1}} \ldots H_{n}^{\omega_{\nu}, t_{\nu}} .
$$

Let $g_{\nu, n, j}(\underline{t}):=\mathrm{i}^{\nu} H_{n}(\underline{t})(j, j)$ be as in (4.3). Using (6.1) we can expand $g_{\nu, n, j}(\underline{t})$ as follows:

$$
g_{\nu, n, j}(\underline{t})=\mathrm{i}^{\nu} \sum_{\underline{\omega} \in\left(\Omega^{*}\right)^{\nu}} c_{\underline{\omega}} g \underline{\underline{\omega}}, \underline{t}(j),
$$

where

$$
g \frac{\omega}{n}, \underline{t}(j):=H \frac{\omega}{n}, \underline{t}(j, j) .
$$

6.2. Approximation of $\boldsymbol{g}_{\boldsymbol{n}}^{\boldsymbol{\omega}}, \underline{\boldsymbol{t}}(\boldsymbol{j})$ by an oscillatory integral. For $j \in\left[n-n^{\gamma}, n+n^{\gamma}\right]$ we approximate the $j$-th diagonal entry $g \frac{\omega}{n}, \underline{t}(j)$ of $H \frac{\omega}{n}, \underline{t}$ by an oscillatory integral of type

$$
\mathfrak{g} \frac{\omega}{n}, \underline{t}(j):=\mathrm{e}^{\mathrm{i} j|\underline{\omega}|_{1}} \int_{0}^{2 \pi} \mathrm{e}^{\mathrm{i} \psi \frac{\omega}{n}, \frac{t}{1}\left(\mathrm{e}^{\mathrm{i} \eta}\right)} b \frac{\omega}{n}, \underline{t}\left(j, \mathrm{e}^{\mathrm{i} \eta}\right) \frac{\mathrm{d} \eta}{2 \pi} .
$$

Here, $\left|\left(\omega_{1}, \ldots, \omega_{\nu}\right)\right|_{1}:=\omega_{1}+\cdots+\omega_{\nu}$, the phase $\psi \frac{\omega}{n, \underline{t}}: \mathbb{T} \rightarrow \mathbb{R}$ is defined in the next subsection, and $b \frac{\omega}{n}, \underline{t}(j, \cdot): \mathbb{T} \rightarrow \mathbb{C}$ is chosen as indicated in Lemma 6.1 below.

6.2.1. Definition of $\psi \frac{\underline{\omega}, \underline{t}, \underline{1}}{\text {. For }}(\underline{\omega}, \underline{t}) \in\left(\Omega^{*}\right)^{\nu} \times \mathbb{R}^{\nu}$ the phase $\psi \frac{\underline{\omega}}{n}, \underline{\underline{t}}$ is given by

$$
\psi\left(\underline{\omega}, \underline{\underline{t}}\left(\mathrm{e}^{\mathrm{i} \xi}\right):=2 a(n) \operatorname{Im}\left(z(\underline{\omega} ; \underline{t}) \mathrm{e}^{\mathrm{i} \xi}\right),\right.
$$

where $z(\underline{\omega} ; \underline{t})$ will be defined by induction on $\nu$.

We first assume $\nu=1$. For $\omega \in \Omega, t \in \mathbb{R}$ we define

$$
z(\omega ; t):=\left(\mathrm{e}^{-\mathrm{i} \omega}-1\right) \mathrm{e}^{-\mathrm{i} t}=-2 \sin \frac{\omega}{2} \mathrm{e}^{\mathrm{i} \pi / 2-\mathrm{i} \omega / 2-\mathrm{i} t} .
$$

Thus the definition of $\psi_{n, 1}^{\omega, t}: \mathbb{T} \rightarrow \mathbb{R}$ is as in [5, (9.10b)]:

$$
\psi_{n, 1}^{\omega, t}\left(\mathrm{e}^{\mathrm{i} \xi}\right):=2 a(n) \operatorname{Im}\left(z(\omega ; t) \mathrm{e}^{\mathrm{i} \xi}\right)=-4 a(n) \sin \frac{\omega}{2} \cos \left(\xi-t-\frac{\omega}{2}\right) .
$$

Moreover, if $\tau_{\omega}: \mathbb{T} \rightarrow \mathbb{T}$ is the translation $\mathrm{e}^{\mathrm{i} \xi} \mapsto \mathrm{e}^{\mathrm{i}(\xi-\omega)}$ and $\psi_{n, 1}^{-\omega, t}:=\psi_{n, 1}^{2 \pi-\omega, t}$ we have the relation

$$
\psi_{n, 1}^{\omega, t}=-\psi_{n, 1}^{-\omega, t} \circ \tau_{\omega} .
$$

Assuming now $\nu \geq 2$ and using induction with respect to $\nu$, we define

$$
z(\underline{\omega} ; \underline{t})=z\left(\underline{\omega}^{\prime} ; \underline{t}^{\prime}\right) \mathrm{e}^{-\mathrm{i} \omega_{\nu}}+z\left(\omega_{\nu} ; t_{\nu}\right),
$$


where

$$
\begin{aligned}
\underline{\omega} & =\left(\underline{\omega}^{\prime}, \omega_{\nu}\right) \in\left(\Omega^{*}\right)^{\nu}=\left(\Omega^{*}\right)^{\nu-1} \times \Omega^{*}, \\
\underline{t} & =\left(\underline{t}^{\prime}, t_{\nu}\right) \in \mathbb{R}^{\nu}=\mathbb{R}^{\nu-1} \times \mathbb{R} .
\end{aligned}
$$

By (6.9) and (6.8) we observe that $\psi \frac{\omega}{n}, \frac{t}{1}$ can also be defined by induction as in [5, (10.7) and (10.6)]:

$$
\psi\left(\frac{\omega, \underline{t}}{n, 1}=\psi \frac{\underline{\omega}^{\prime}, \underline{t}^{\prime}}{n, 1^{\prime}} \circ \tau_{\omega_{\nu}}+\psi_{n, 1}^{\omega_{\nu}, t_{\nu}}=\left(\psi_{n, 1^{\prime}, \underline{t}^{\prime}}-\psi_{n, 1}^{-\omega_{\nu}, t_{\nu}}\right) \circ \tau_{\omega_{\nu}},\right.
$$

6.2.2. Approximation of $g_{\frac{\omega}{n}, \underline{t}}(j)$ by $\mathfrak{g}_{n}^{\underline{\omega}, \underline{t}}(j)$.

Lemma 6.1. Let $t_{0}>0$ and $0<\varepsilon<\frac{1}{8}$ be fixed. Let $\mathfrak{g}^{\underline{\omega}} \frac{\underline{t}}{n}(j)$ be the oscillatory integral defined by (6.4) for $|j-n| \leq n^{\gamma}$ with $\psi \frac{\omega, t}{n, 1}$ as in (6.5). If $\hat{C}=\hat{C}\left(t_{0}, \varepsilon\right)>0$ is large enough, then for any $\nu \in \mathbb{N}^{*},(\underline{\omega}, \underline{t}) \in\left(\Omega^{*}\right)^{\nu} \times\left[-t_{0}, t_{0}\right]^{\nu}, n \geq \nu^{\frac{1}{\varepsilon}}$, and $j \in\left[n-n^{\gamma}, n+n^{\gamma}\right]$ we can choose $b \frac{\omega}{n}, \underline{t}(j, \cdot): \mathbb{T} \rightarrow \mathbb{C}$ in (6.4) such that $\mathfrak{g} \frac{\omega}{n}, \underline{t}(j)$ satisfies

$$
\sup _{|j-n| \leq n \gamma}\left|g \frac{\omega}{n}, \underline{t}(j)-\mathfrak{g} \frac{\omega}{n}, \underline{t}(j)\right| \leq \hat{C} n^{-\gamma+4 \varepsilon},
$$

with

$$
\begin{aligned}
& \left|b \frac{\omega}{n}, \underline{t}\left(j, \mathrm{e}^{\mathrm{i} \eta}\right)\right|=1, \\
& \left\|b \frac{\omega}{n}, \underline{t}(j, \cdot)\right\|_{\mathrm{C}^{2}(\mathbb{T})} \leq \hat{C} n^{4 \varepsilon}, \\
& \left\|\partial_{t_{\nu}} b \frac{\omega}{n}, \underline{t}(j, \cdot)\right\|_{\mathrm{C}^{0}(\mathbb{T})} \leq \hat{C} n^{\varepsilon}, \quad \nu \geq 2 .
\end{aligned}
$$

Proof. See Section 8.1, in particular Section 8.1.1 and (8.7) for the actual choice of $b \frac{\omega, t}{n}(j, \cdot)$.

6.3. Properties of $z(\underline{\omega} ; \underline{t})$. Let $z(\underline{\omega} ; \underline{t}), \underline{\omega} \in\left(\Omega^{*}\right)^{\nu}, \underline{t} \in \mathbb{R}^{\nu}$ be as in Section 6.2.1. We write

$$
z(\underline{\omega} ; \underline{t})=|z(\underline{\omega} ; \underline{t})| \mathrm{e}^{\mathrm{i} \alpha(\underline{\omega} ; \underline{t})},
$$

where $0 \leq \alpha(\underline{\omega} ; \underline{t})<2 \pi$ is the argument of $z(\underline{\omega} ; \underline{t})$. If $\nu \geq 2$ we write $\underline{\omega}=\left(\underline{\omega}^{\prime}, \omega_{\nu}\right) \in\left(\Omega^{*}\right)^{\nu-1} \times \Omega^{*}$, $\underline{t}=\left(\underline{t}^{\prime}, t_{\nu}\right) \in \mathbb{R}^{\nu-1} \times \mathbb{R}$ as in (6.10) and define

$$
\begin{aligned}
\hat{z}\left(\underline{\omega} ; \underline{t}^{\prime}\right) & :=\frac{z\left(\underline{\omega}^{\prime} ; \underline{t}^{\prime}\right)}{2 \sin \left(\omega_{\nu} / 2\right)}, \\
\hat{\alpha}\left(\underline{\omega} ; \underline{t}^{\prime}\right) & :=\alpha\left(\underline{\omega}^{\prime} ; \underline{t}^{\prime}\right)-\omega_{\nu} / 2-\pi / 2 .
\end{aligned}
$$

For the next lemma we also introduce the function $\mathfrak{h}: \mathbb{R} \times \mathbb{C} \rightarrow \mathbb{R}_{+}$by

$$
\mathfrak{h}(t, z):=\sqrt{4|z| \sin ^{2}(t / 2)+(1-|z|)^{2}} .
$$

Lemma 6.2. We assume $\nu \geq 2$. Then for $\underline{\omega}=\left(\underline{\omega}^{\prime}, \omega_{\nu}\right) \in\left(\Omega^{*}\right)^{\nu-1} \times \Omega^{*}$ and $\underline{t}=\left(\underline{t}^{\prime}, t_{\nu}\right) \in \mathbb{R}^{\nu-1} \times \mathbb{R}$ we have the relation

$$
|z(\underline{\omega} ; \underline{t})|=2 \sin \left(\omega_{\nu} / 2\right) \mathfrak{h}\left(t_{\nu}+\hat{\alpha}\left(\underline{\omega} ; \underline{t}^{\prime}\right), \hat{z}\left(\underline{\omega} ; \underline{t}^{\prime}\right)\right),
$$

where $\mathfrak{h}$ is given by (6.17), $\hat{z}\left(\underline{\omega} ; \underline{t}^{\prime}\right)$ by (6.15), and $\hat{\alpha}\left(\underline{\omega} ; \underline{t}^{\prime}\right)$ by (6.16). Moreover,

$$
|z(\underline{\omega} ; \underline{t})| \geq \frac{2 \sin \left(\omega_{\nu} / 2\right)}{\pi}\left|t_{\nu}+\hat{\alpha}\left(\underline{\omega} ; \underline{t}^{\prime}\right)\right|_{2 \pi}
$$

where $|t|_{2 \pi}:=\operatorname{dist}(t, 2 \pi \mathbb{Z})$ and

$$
\left|\partial_{t_{\nu}} \mathrm{e}^{\mathrm{i} \alpha(\underline{\omega} ; \underline{t})}\right| \leq \frac{6}{|z(\underline{\omega} ; \underline{t})|} .
$$

Proof. The proof consists of four steps. 
Step 1. We first claim that for $t \in \mathbb{R}$ and $z \in \mathbb{C}$

$$
|| z\left|-\mathrm{e}^{-\mathrm{i} t}\right|=\mathfrak{h}(t, z)
$$

Indeed, the left hand side of (6.21) is

$$
\sqrt{(|z|-\cos t)^{2}+\sin ^{2} t}=\sqrt{2|z|(1-\cos t)+(1-|z|)^{2}}
$$

which is $\mathfrak{h}(t, z)$ defined by (6.17) due to $1-\cos t=2 \sin ^{2}(t / 2)$.

Step 2. Now we will show (6.18). Combining (6.6), (6.9) with (6.14) we obtain

$$
z(\underline{\omega} ; \underline{t})=\left|z\left(\underline{\omega} ; \underline{t}^{\prime}\right)\right| \mathrm{e}^{\mathrm{i} \alpha\left(\underline{\omega}^{\prime} ; \underline{t}^{\prime}\right)-\mathrm{i} \omega_{\nu}}-2 \sin \left(\omega_{\nu} / 2\right) \mathrm{e}^{-\mathrm{i}\left(t_{\nu}+\omega_{\nu} / 2-\pi / 2\right)} .
$$

Using (6.15) and (6.16) in the right hand side of (6.22) we find

$$
z(\underline{\omega} ; \underline{t})=2 \sin \left(\omega_{\nu} / 2\right)\left(\left|\hat{z}\left(\underline{\omega} ; \underline{t}^{\prime}\right)\right|-\mathrm{e}^{-\mathrm{i}\left(t_{\nu}+\hat{\alpha}\left(\underline{\omega} ; \underline{t}^{\prime}\right)\right)}\right) \mathrm{e}^{\mathrm{i} \alpha\left(\underline{\omega}^{\prime} ; \underline{t}^{\prime}\right)-\mathrm{i} \omega_{\nu}},
$$

hence

$$
|z(\underline{\omega} ; \underline{t})|=2 \sin \left(\omega_{\nu} / 2\right)|| \hat{z}\left(\underline{\omega} ; \underline{t}^{\prime}\right)\left|-\mathrm{e}^{-\mathrm{i}\left(t_{\nu}+\hat{\alpha}\left(\underline{\omega} ; \underline{t}^{\prime}\right)\right)}\right| .
$$

Denoting $z:=\hat{z}\left(\underline{\omega} ; \underline{t}^{\prime}\right), t:=t_{\nu}+\hat{\alpha}\left(\underline{\omega} ; \underline{t}^{\prime}\right)$ and using (6.21) we can express the right hand side of (6.23) in the form

$$
2 \sin \left(\omega_{\nu} / 2\right)|| z\left|-\mathrm{e}^{-\mathrm{i} t}\right|=2 \sin \left(\omega_{\nu} / 2\right) \mathfrak{h}(t, z),
$$

which completes the proof of (6.18).

Step 3. Here we will show (6.19). Combining

$$
\mathfrak{h}(t, z) \geq \sqrt{\left(4|z|+(1-|z|)^{2}\right) \sin ^{2}(t / 2)}=(1+|z|)|\sin (t / 2)|
$$

with (6.18) we obtain

$$
|z(\underline{\omega} ; \underline{t})| \geq 2 \sin \left(\omega_{\nu} / 2\right)\left|\sin \frac{1}{2}\left(t_{\nu}+\hat{\alpha}\left(\underline{\omega} ; \underline{t}^{\prime}\right)\right)\right| .
$$

Thus (6.19) follows from $|\sin (t / 2)| \geq \frac{1}{\pi}|t|_{2 \pi}$.

Step 4. Finally we show (6.20). We begin by writing

$$
\partial_{t_{\nu}} \frac{z(\underline{\omega} ; \underline{t})}{|z(\underline{\omega} ; \underline{t})|}=\frac{1}{|z(\underline{\omega} ; \underline{t})|}\left(\partial_{t_{\nu}} z(\underline{\omega} ; \underline{t})-\frac{z(\underline{\omega} ; \underline{t})}{|z(\underline{\omega} ; \underline{t})|} \partial_{t_{\nu}}|z(\underline{\omega} ; \underline{t})|\right) .
$$

Since $\left|\partial_{t_{\nu}} z(\underline{\omega} ; \underline{t})\right|=\left|\partial_{t_{\nu}} z\left(\omega_{\nu} ; t_{\nu}\right)\right|=2 \sin \left(\omega_{\nu} / 2\right) \leq 2$, the proof of $\underline{6.20}$ is completed if we show

$$
\left|\partial_{t_{\nu}}\right| z(\underline{\omega} ; \underline{t})|| \leq 4 \text {. }
$$

However using (6.18) we obtain

$$
\partial_{t_{\nu}}|z(\underline{\omega} ; \underline{t})|=2 \sin \left(\omega_{\nu} / 2\right) \partial_{t_{\nu}} \mathfrak{h}\left(t_{\nu}+\hat{\alpha}\left(\underline{\omega} ; \underline{t}^{\prime}\right), \hat{z}\left(\underline{\omega} ; \underline{t}^{\prime}\right)\right)
$$

Then using (6.24) we have

$$
\left|\partial_{t} \mathfrak{h}(t, z)\right|=\frac{|z||\sin t|}{\mathfrak{h}(t, z)} \leq \frac{|z||\sin t|}{(1+|z|)|\sin (t / 2)|}
$$

and (6.25) results from the inequality $|\sin t| \leq 2\left|\sin \frac{t}{2}\right|$. 


\section{Proof of Proposition 4.1}

7.1. First reductions. In this section $0<\varepsilon<\frac{1}{8}$ and $t_{0}>0$ are fixed. As in (6.2) we denote by $H \frac{\omega}{n}, \underline{t}$ the operator $H_{n}^{\omega_{1}, t_{1}} \ldots H_{n}^{\omega_{\nu}, t_{\nu}}$ for $(\underline{\omega}, \underline{t}) \in\left(\Omega^{*}\right)^{\nu} \times \mathbb{R}^{\nu}$ and by $g g_{n}^{\underline{\omega}}, \underline{t}(j)$ its $j$-th diagonal entry.

We first observe that instead of (4.7) it suffices to prove the estimates

$$
\begin{gathered}
\left|\int_{\Delta_{1}} g \frac{\omega}{n}, \underline{t}(j) \mathrm{d} t_{2}\right| \leq C n^{-\gamma+5 \varepsilon} \quad(\nu=2), \\
\int_{\Delta_{2}}\left|\int_{\Delta_{1}} g_{\frac{\omega}{n}, \underline{t}}(j) \mathrm{d} t_{\nu}\right| \mathrm{d} t_{\nu-1} \leq C n^{-\gamma+5 \varepsilon} \quad\left(3 \leq \nu \leq n^{\varepsilon}\right)
\end{gathered}
$$

for any intervals $\Delta_{1}, \Delta_{2} \subset\left[-t_{0}, t_{0}\right],(\underline{\omega}, \underline{t}) \in\left(\Omega^{*}\right)^{\nu} \times\left[-t_{0}, t_{0}\right]^{\nu}$, and $j \in\left[n-n^{\gamma}, n+n^{\gamma}\right]$.

Further on, $\mathfrak{g}^{\underline{\omega}, \underline{t}}(j)$ is given by (6.4) with $b_{n}^{\underline{\omega}, \underline{t}}(j, \cdot)$ as in Lemma 6.1] $|j-n| \leq n^{\gamma},(\underline{\omega}, \underline{t}) \in$ $\left(\Omega^{*}\right)^{\nu} \times\left[-t_{0}, t_{0}\right]^{\nu}$, and $2 \leq \nu \leq n^{\varepsilon}$. Then due to Lemma 6.1, instead of proving estimates (7.1) it suffices to prove these ones:

$$
\begin{gathered}
\left|\int_{\Delta_{1}} \mathfrak{g} \frac{\omega}{n}, \underline{t}(j) \mathrm{d} t_{2}\right| \leq C n^{-\gamma+5 \varepsilon} \quad(\nu=2), \\
\int_{\Delta_{2}}\left|\int_{\Delta_{1}} \mathfrak{g} \frac{\omega}{n}, \underline{t}(j) \mathrm{d} t_{\nu}\right| \mathrm{d} t_{\nu-1} \leq C n^{-\gamma+5 \varepsilon} \quad\left(3 \leq \nu \leq n^{\varepsilon}\right) .
\end{gathered}
$$

To estimate $\int_{\Delta_{1}} \mathfrak{g} \frac{\omega}{n}, \underline{t}(j) \mathrm{d} t_{\nu}$ it suffices to consider the following two cases:

1) $\Delta_{1} \subset\left[-n^{-\gamma}, n^{-\gamma}\right]+2 \pi \mathbb{Z}-\hat{\alpha}\left(\underline{\omega} ; \underline{t}^{\prime}\right)$,

2) $\Delta_{1} \subset\left[n^{-\gamma}, 2 \pi-n^{-\gamma}\right]+2 \pi \mathbb{Z}-\hat{\alpha}\left(\underline{\omega} ; \underline{t}^{\prime}\right)$,

where $\hat{\alpha}\left(\underline{\omega} ; \underline{t}^{\prime}\right):=\alpha\left(\underline{\omega}^{\prime} ; \underline{t}^{\prime}\right)-\omega_{\nu} / 2-\pi / 2$ as in (6.16).

7.2. Case 1). We assume $\nu \geq 2$. The definition of $H \frac{\omega}{n}, \underline{t}$ shows that its diagonal entries satisfy $\left|g g_{n}^{\frac{\omega}{n}}(j)\right| \leq 1$. Then, since $|j-n| \leq n^{\gamma}$, estimate (6.12) from Lemma 6.1 applies and gives

$$
\left|\mathfrak{g} \frac{\omega}{n}, \underline{t}(j)\right| \leq C_{1} n^{4 \varepsilon}
$$

for some constant $C_{1}>0$. Since $\Delta_{1}$ is a subinterval of $\left[-t_{0}, t_{0}\right]$ satisfying

$$
\Delta_{1} \subset\left[-n^{-\gamma}, n^{-\gamma}\right]+2 \pi \mathbb{Z}-\hat{\alpha}\left(\underline{\omega} ; \underline{t}^{\prime}\right),
$$

its length satisfies $\left|\Delta_{1}\right| \leq 2 n^{-\gamma}$ and using (7.3) we get the estimate

$$
\left|\int_{\Delta_{1}} \mathfrak{g}_{n}^{\underline{\omega}, t}(j) \mathrm{d} t_{\nu}\right| \leq C_{0} n^{-\gamma+4 \varepsilon}
$$

for some constant $C_{0}>0$. Thus, in case 1) the proof of (7.2) is completed.

7.3. Case 2). We now assume $\Delta_{1}$ is a subinterval of $\left[-t_{0}, t_{0}\right]$ satisfying

$$
\Delta_{1} \subset\left[n^{-\gamma}, 2 \pi-n^{-\gamma}\right]+2 \pi \mathbb{Z}-\hat{\alpha}\left(\underline{\omega} ; \underline{t}^{\prime}\right) .
$$

If $t_{\nu} \in \Delta_{1}$, then $\left|t_{\nu}+\hat{\alpha}\left(\underline{\omega} ; \underline{t}^{\prime}\right)\right|_{2 \pi} \geq n^{-\gamma}$ and $|z(\underline{\omega} ; \underline{t})| \geq(2 / \pi) \sin (\pi / N) n^{-\gamma}$ by estimate (6.19) from Lemma 6.2. In particular, $z(\underline{\omega} ; \underline{t}) \neq 0$.

7.3.1. Use of the stationary phase formula. Writing $z(\underline{\omega} ; \underline{t})=|z(\underline{\omega} ; \underline{t})| \mathrm{e}^{\mathrm{i} \alpha(\underline{\underline{\omega}} ; \underline{t})}$ in (6.5) we find

$$
\psi\left(\underline{\omega}, \underline{\underline{t}},\left(\mathrm{e}^{\mathrm{i} \xi}\right)=2 a(n)|z(\underline{\omega} ; \underline{t})| \sin (\xi+\alpha(\underline{\omega} ; \underline{t})) .\right.
$$

Using this expression in (6.4) we get

$$
\mathfrak{g} \frac{\omega}{n}, \underline{t}(j)=\mathrm{e}^{\mathrm{i} j|\underline{\omega}|_{1}} \int_{0}^{2 \pi} \mathrm{e}^{2 \mathrm{i} a(n)|z(\underline{\omega} ; \underline{\underline{t}})| \sin (\eta+\alpha(\underline{\omega} ; \underline{t}))} b \underline{\omega}, \underline{\underline{t}}\left(j, \mathrm{e}^{\mathrm{i} \eta}\right) \frac{\mathrm{d} \eta}{2 \pi} .
$$


This oscillatory integral is of the type $\mathcal{J}\left(b, \mu, \eta_{0}\right)$ considered in Lemma 3.1 for $b=b \frac{\omega}{n}, \underline{t}(j, \cdot)$, $\mu=2 a(n)|z(\underline{\omega} ; \underline{t})|$, and $\eta_{0}=\pi / 2-\alpha(\underline{\omega} ; \underline{t})$. Since $z(\underline{\omega} ; \underline{t}) \neq 0$ the stationary phase formula (3.2) applies:

with

$$
\mathrm{e}^{-\mathrm{i} j|\underline{\omega}|_{1}} \mathfrak{g} \frac{\omega}{n}, \underline{t}(j)=\sum_{\kappa= \pm 1} \frac{\mathrm{e}^{\mathrm{i} \kappa(2 a(n)|z(\underline{\omega} ; \underline{t})|-\pi / 4)}}{2 \sqrt{\pi a(n)|z(\underline{\omega} ; \underline{t})|}} b \frac{\underline{\omega}, \underline{t}}{n}\left(j, \kappa \mathrm{ie}^{-\mathrm{i} \alpha(\underline{\omega} ; \underline{t})}\right)+\mathfrak{r} \frac{\omega}{n}, \underline{t}(j)
$$

with

$$
\left|\mathfrak{r} \frac{\omega}{n}, \underline{t}(j)\right| \leq \frac{C_{0}}{a(n)|z(\underline{\omega} ; \underline{t})|}\left\|b \frac{\omega}{n}, \underline{t}(j, \cdot)\right\|_{\mathrm{C}^{2}(\mathbb{T})} .
$$

Integrating (7.8a ) we find

$$
\mathrm{e}^{-\mathrm{i} j|\underline{\underline{\omega}}|_{1}} \int_{\Delta_{1}} \mathfrak{g} \underline{\underline{\omega}}, \underline{\underline{t}}(j) \mathrm{d} t_{\nu}=\mathrm{e}^{-\mathrm{i} \pi / 4} \mathcal{J} \frac{\omega}{n, \underline{t}^{\prime}}\left(\Delta_{1}\right)+\mathrm{e}^{\mathrm{i} \pi / 4} \mathcal{J} \frac{\omega}{n, j \underline{t}^{\prime}}\left(\Delta_{1}\right)+\int_{\Delta_{1}} \mathfrak{r} \frac{\omega}{n}, \underline{t}(j) \mathrm{d} t_{\nu}
$$

where

$$
\mathcal{J}_{n, j, \pm}^{\underline{\omega}, \underline{t}^{\prime}}\left(\Delta_{1}\right):=\int_{\Delta_{1}} \frac{\mathrm{e}^{ \pm 2 \mathrm{i} a(n)|z(\underline{\omega} ; \underline{\underline{\omega}})|}}{2 \sqrt{\pi a(n)|z(\underline{\omega} ; \underline{t})|}} b \frac{\omega}{n}, \underline{t}\left(j, \pm \mathrm{ie}^{-\mathrm{i} \alpha(\underline{\omega} ; \underline{t})}\right) \mathrm{d} t_{\nu} .
$$

7.3.2. Remainder estimate. We claim that the remainder in (7.9) can be estimated by

$$
\left|\int_{\Delta_{1}} \mathfrak{r} \frac{\omega}{n}, \underline{t}(j) \mathrm{d} t_{\nu}\right| \leq C n^{-\gamma+5 \varepsilon}
$$

Indeed, (H1a), (6.19) and (6.13b) allow us to derive from estimate (7.8b) of $\left|\mathfrak{r} \frac{\omega}{n}, \underline{t}(j)\right|$ this new one

and to get (7.11) it suffices to observe that

$$
|\mathfrak{r}, \underline{\underline{\omega}}, \underline{t}(j)| \leq \frac{C n^{4 \varepsilon}}{n^{\gamma}\left|t_{\nu}+\hat{\alpha}\left(\underline{\omega} ; \underline{t}^{\prime}\right)\right|_{2 \pi}}
$$

$$
\int_{\Delta_{1}} \frac{\mathrm{d} t_{\nu}}{\left|t_{\nu}+\hat{\alpha}\left(\underline{\omega} ; \underline{t}^{\prime}\right)\right|_{2 \pi}} \leq 2 \int_{n^{-\gamma}}^{\pi} \frac{\mathrm{d} t}{t}=\mathrm{O}(\ln n) .
$$

To complete the proof of (17.2) in case 2) we will prove the following estimates:

$$
\begin{aligned}
\left|\mathcal{J}_{n, j, \pm}^{\underline{\omega}, t^{\prime}}\left(\Delta_{1}\right)\right| & \leq C n^{-\gamma+2 \varepsilon} \quad(\nu=2), \\
\int_{\Delta_{2}}\left|\mathcal{J}_{n, j, \pm}^{\underline{\omega}, t^{\prime}}\left(\Delta_{1}\right)\right| \mathrm{d} t_{\nu-1} & \leq C n^{-\gamma+2 \varepsilon} \quad\left(3 \leq \nu \leq n^{\varepsilon}\right) .
\end{aligned}
$$

According to (7.9) these estimates together with the remainder estimate (7.11) actually imply (7.2) in case 2).

7.3.3. Transformation of $\mathcal{J} \frac{\omega}{n, t^{\prime}}, \pm\left(\Delta_{1}\right)$. If the new variable $t=t_{\nu}+\hat{\alpha}\left(\underline{\omega} ; \underline{t}^{\prime}\right)$ is introduced, then (6.18) becomes $|z(\underline{\omega} ; \underline{t})|=2 \sin \left(\omega_{\nu} / 2\right) \mathfrak{h}\left(t, \hat{z}\left(\underline{\omega} ; \underline{t}^{\prime}\right)\right)$ and (7.10) takes the form

$$
\mathcal{J}_{n, j, \pm}^{\underline{\omega}, \underline{t}^{\prime}}\left(\Delta_{1}\right)=\int_{\Delta_{1}+\hat{\alpha}\left(\underline{\omega} ; \underline{t}^{\prime}\right)} \frac{\mathrm{e}^{ \pm \mathrm{i} \tilde{\mu}_{n}\left(\omega_{\nu}\right) \mathfrak{h}\left(t, \hat{z}\left(\underline{\omega} ; \underline{t}^{\prime}\right)\right)}}{\sqrt{2 \pi \tilde{\mu}_{n}\left(\omega_{\nu}\right) \mathfrak{h}\left(t, \hat{z}\left(\underline{\omega} ; \underline{t}^{\prime}\right)\right)}} b \frac{\omega}{\underline{\omega}, \underline{t}^{\prime}, \pm}(t) \mathrm{d} t
$$

where

$$
\begin{aligned}
& \tilde{\mu}_{n}\left(\omega_{\nu}\right):=4 a(n) \sin \left(\omega_{\nu} / 2\right),
\end{aligned}
$$

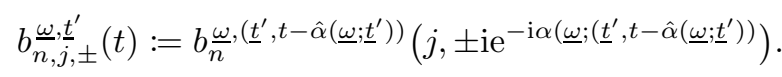

Our goal is to get (7.12). We first observe that in the case $\nu=2$ we have $\left|z\left(\underline{\omega}^{\prime} ; \underline{t}^{\prime}\right)\right|=\left|z\left(\omega_{1} ; t_{1}\right)\right|=$ $2 \sin \left(\omega_{1} / 2\right)>0$ for $\omega_{1} \in \Omega^{*}$, hence $\hat{z}\left(\underline{\omega} ; \underline{t}^{\prime}\right) \neq 0$. In the case $\nu \geq 3$ we have to estimate the integral $\left.\int_{\Delta_{2}} \mid \mathcal{J}_{n, j, \pm}^{\omega}, t^{\prime}, \Delta_{1}\right) \mid \mathrm{d} t_{\nu-1}$ and we observe that in this integral we can forget the $t_{\nu-1}$ such that $\hat{z}\left(\underline{\omega} ; \underline{t}^{\prime}\right)=0$, or equivalently, $z\left(\underline{\omega}^{\prime} ; \underline{t}^{\prime}\right)=0$ because there are only finitely many. By (6.19) 
they indeed satisfy $t_{\nu-1} \in 2 \pi \mathbb{Z}-\hat{\alpha}\left(\underline{\omega}^{\prime} ; \underline{t}^{\prime \prime}\right)$ where $\underline{t}^{\prime}=\left(\underline{t}^{\prime \prime}, t_{\nu-1}\right)$. So, we henceforth assume $\hat{z}\left(\underline{\omega} ; \underline{t}^{\prime}\right) \neq 0$ when estimating $\mathcal{J}_{n, j, \pm}^{\underline{t^{\prime}}}\left(\Delta_{1}\right)$.

Our next step is to write (7.13a) as an integral of the type considered in Section 5 For this purpose we denote by $\zeta \equiv \zeta(\hat{z})$ the nonnegative number associated to $\hat{z} \in \mathbb{C}^{*}$ by

$$
\zeta:=\left.|| \hat{z}\right|^{-1 / 2}-|\hat{z}|^{1 / 2} \mid .
$$

Since $(1-|\hat{z}|)^{2}=\zeta^{2}|\hat{z}|$, we can write $\mathfrak{h}(t, \hat{z}), \hat{z} \neq 0$ as follows:

$$
\mathfrak{h}(t, \hat{z}):=\sqrt{4|\hat{z}| \sin ^{2}(t / 2)+(1-|\hat{z}|)^{2}}=\sqrt{|\hat{z}|} \sqrt{4 \sin ^{2}(t / 2)+\zeta^{2}} .
$$

Then for $\mu>0, \zeta \geq 0$, and $\Delta_{1}^{\prime}$ a bounded interval we introduce the integral

$$
\tilde{\mathcal{J}} \frac{\tilde{\omega}, \underline{t}^{\prime}}{n, j, \pm}\left(\zeta, \mu, \Delta_{1}^{\prime}\right):=\frac{1}{\sqrt{2 \pi \mu}} \int_{\Delta_{1}^{\prime}} \frac{\mathrm{e}^{ \pm \mathrm{i} \mu \sqrt{4 \sin ^{2}(t / 2)+\zeta^{2}}}}{\left(4 \sin ^{2}(t / 2)+\zeta^{2}\right)^{1 / 4}} b \frac{\omega,, t^{\prime}}{n, \pm}(t) \mathrm{d} t .
$$

Due to (7.15) we have

where $\Delta_{1}^{\prime}=\Delta_{1}+\hat{\alpha}\left(\underline{\omega} ; \underline{t}^{\prime}\right)$ and

$$
\mathcal{J}_{n, j, \pm}^{\underline{\omega}, \underline{t}^{\prime}}\left(\Delta_{1}\right)=\tilde{\mathcal{J}}_{n, j, \pm}^{\underline{\omega}, \underline{t}^{\prime}}\left(\zeta, \mu, \Delta_{1}^{\prime}\right)
$$

$$
\begin{aligned}
& \zeta \equiv \zeta\left(\underline{\omega} ; \underline{t}^{\prime}\right):=\left.|| \hat{z}\left(\underline{\omega} ; \underline{t}^{\prime}\right)\right|^{1 / 2}-\left|\hat{z}\left(\underline{\omega} ; \underline{t}^{\prime}\right)\right|^{-1 / 2} \mid \\
& \mu \equiv \mu_{n}\left(\underline{\omega} ; \underline{t}^{\prime}\right):=\tilde{\mu}_{n}\left(\omega_{\nu}\right)\left|\hat{z}\left(\underline{\omega} ; \underline{t^{\prime}}\right)\right|^{1 / 2}
\end{aligned}
$$

Thus it remains to investigate $\tilde{\mathcal{J}} \frac{\omega, t^{\prime}, \pm}{j^{\prime}}\left(\zeta, \mu, \Delta_{1}^{\prime}\right)$ with $\zeta$ and $\mu$ as in (7.17b) , and (7.17c), and

$$
\Delta_{1}^{\prime} \subset\left[n^{-\gamma}, 2 \pi-n^{-\gamma}\right]+2 \pi \mathbb{Z} .
$$

7.3.4. Estimate of $\tilde{\mathcal{J}}_{n, j, t^{\prime}}^{\omega}\left(\zeta, \mu, \Delta_{1}^{\prime}\right)$. In this section $\zeta$ and $\mu$ are given by (7.17b) and (7.17c) and $\Delta_{1}^{\prime}$ is an interval satisfying (7.18). We claim that there is a constant $C>0$ such that

$$
\left|\tilde{\mathcal{J}}_{n, j, \pm}^{\underline{\omega}, \underline{t}^{\prime}}\left(\zeta, \mu, \Delta_{1}^{\prime}\right)\right| \leq C \frac{n^{2 \varepsilon}}{a(n)}\left(1+\left|\hat{z}\left(\underline{\omega} ; \underline{t}^{\prime}\right)\right|^{-3 / 4}\right) .
$$

For this purpose we first observe that $\tilde{\mathcal{J}}_{n, j, t^{\prime}}^{\omega}\left(\zeta, \mu, \Delta_{1}^{\prime}\right)$ coincides with the oscillatory integral $\mathcal{J}\left(b, t_{1}, t_{2}, \zeta, \pm \mu\right)$ defined in (5.1) for $b=\frac{1}{\sqrt{2 \pi \mu}} b \frac{\underline{\omega}, \underline{t}^{\prime}, \pm}{(t)}$ and $\Delta_{1}^{\prime}=\left[t_{1}, t_{2}\right]$. Since $\mu=\mu_{n}\left(\underline{\omega} ; \underline{t}^{\prime}\right)>0$, Lemma 5.1 applies and gives

$$
\left|\tilde{\mathcal{J}}_{n, j, \pm}^{\omega, t^{\prime}}\left(\zeta, \mu, \Delta_{1}^{\prime}\right)\right| \leq C \frac{1+\zeta^{1 / 2}}{\mu} \mathcal{M}\left(b \frac{\omega, t^{\prime}}{j_{j}}, \Delta_{1}^{\prime}\right)
$$

Since

$$
\frac{1+\zeta^{1 / 2}}{\mu} \leq \frac{1+\left|\hat{z}\left(\underline{\omega} ; \underline{t}^{\prime}\right)\right|^{1 / 4}+\left|\hat{z}\left(\underline{\omega} ; \underline{t}^{\prime}\right)\right|^{-1 / 4}}{4 a(n) \sin \left(\omega_{\nu} / 2\right)\left|\hat{z}\left(\underline{\omega} ; \underline{t}^{\prime}\right)\right|^{1 / 2}} \leq \frac{1+\left|\hat{z}\left(\underline{\omega} ; \underline{t}^{\prime}\right)\right|^{-3 / 4}}{2 a(n) \sin \left(\omega_{\nu} / 2\right)}
$$

we deduce (7.19) from (17.20) if we show the estimate

$$
\mathcal{M}\left(b \frac{\omega}{\underline{\omega}, t^{\prime}, \pm}, \Delta_{1}^{\prime}\right) \leq C n^{\varepsilon} \ln n
$$

However, using definition (7.13c) we see that relation (6.13a) from Lemma 6.1 gives

$$
\left|b \frac{\omega}{n, j, \pm} \underline{t}^{\prime}(t)\right|=1 \text {. }
$$

Moreover, by estimate (6.13c) from Lemma 6.1] and estimates (6.20) and (6.19) from Lemma 6.2 we have

$$
\left|\partial_{t} b \frac{\omega}{n, \underline{t}^{\prime}, \pm}(t)\right| \leq\left\|\partial_{t_{\nu}} b \frac{\omega}{n}, \underline{t}(j, \cdot)\right\|_{\mathrm{C}^{0}(\mathbb{T})}\left\|\partial_{t_{\nu}} \mathrm{e}^{-\mathrm{i} \alpha(\underline{\omega} ; \underline{\underline{t}})}\right\|_{\mathrm{C}^{0}(\mathbb{T})} \leq \hat{C} n^{\varepsilon} \frac{6}{|z(\underline{\omega} ; \underline{t})|} \leq C^{\prime} n^{\varepsilon}\left(1+\frac{1}{|t|_{2 \pi}}\right) .
$$


Thus $\mathcal{M}\left(b \frac{\omega, t^{\prime}}{n, j, \pm}, \Delta_{1}^{\prime}\right):=\sup _{\Delta_{1}^{\prime}}\left|b \frac{\omega, t^{\prime}, \pm}{n, \pm}(t)\right|+\int_{\Delta_{1}^{\prime}}\left|\partial_{t} b \frac{\omega}{n, t_{j}^{\prime}}(t)\right| \mathrm{d} t$ can be estimated by

$$
\mathcal{M}\left(b \frac{\omega}{n, t_{j}^{\prime}, \pm}, \Delta_{1}^{\prime}\right) \leq 1+C^{\prime} n^{\varepsilon}\left(2 \pi+\int_{\Delta_{1}^{\prime}} \frac{\mathrm{d} t}{|t|_{2 \pi}}\right)=1+2 C^{\prime} n^{\varepsilon}\left(\pi+\int_{n^{-\gamma}}^{\pi} \frac{\mathrm{d} t}{t}\right)=\mathrm{O}\left(n^{\varepsilon} \ln n\right) .
$$

7.3.5. End of the proof of estimates (7.2) in case 2). Due to (7.17), (7.19), (6.15) and (H1a), there is a constant $C>0$ such that

$$
\left|\mathcal{J}_{n, j, t^{\prime}}\left(\Delta_{1}\right)\right| \leq C n^{-\gamma+2 \varepsilon}\left(1+\left|z\left(\underline{\omega}^{\prime} ; \underline{t}^{\prime}\right)\right|^{-3 / 4}\right) .
$$

If $\nu=2$, then $\underline{\omega}^{\prime}=\omega_{1} \in \Omega^{*}$ and $\left|z\left(\underline{\omega}^{\prime} ; \underline{t}^{\prime}\right)\right|=2 \sin \left(\omega_{1} / 2\right) \geq 2 \sin (\pi / N)>0$ and it is clear that (7.22) implies (7.12a). We then get (17.2a) using (7.12a) and (7.11). This proves assertion (a) in Proposition 4.1

If $\nu \geq 3$, then $\underline{\omega}^{\prime}=\left(\underline{\omega}^{\prime \prime}, \omega_{\nu-1}\right) \in\left(\Omega^{*}\right)^{\nu-2} \times \Omega^{*}$ and $\underline{t}^{\prime}=\left(\underline{t}^{\prime \prime}, t_{\nu-1}\right) \in \mathbb{R}^{\nu-2} \times \mathbb{R}$. If

$$
\hat{\alpha}\left(\underline{\omega}^{\prime}, \underline{t}^{\prime \prime}\right)=\alpha\left(\underline{\omega}^{\prime \prime}, \underline{t}^{\prime \prime}\right)-\omega_{\nu-1} / 2-\pi / 2
$$

then $t_{\nu-1}+\hat{\alpha}\left(\underline{\omega}^{\prime} ; \underline{t}^{\prime \prime}\right) \notin 2 \pi \mathbb{Z}$ implies $\hat{z}\left(\underline{\omega} ; \underline{t}^{\prime}\right) \neq 0$ and

$$
\mathcal{J}_{n, j, \pm}^{\underline{\omega}, t^{\prime}}\left(\Delta_{1}\right) \leq C^{\prime} n^{-\gamma+2 \varepsilon}\left(1+\left|t_{\nu-1}+\hat{\alpha}\left(\underline{\omega}^{\prime}, \underline{t}^{\prime \prime}\right)\right|_{2 \pi}^{-3 / 4}\right)
$$

and therefore

$$
\int_{\Delta_{2}} \mathcal{J}_{n, j, \pm}^{\underline{\omega}, \underline{t}^{\prime}}\left(\Delta_{1}\right) \mathrm{d} t_{\nu-1} \leq C^{\prime} n^{-\gamma+2 \varepsilon} \int_{\Delta_{2}-\hat{\alpha}\left(\underline{\omega}^{\prime}, \underline{t}^{\prime \prime}\right)}\left(1+|t|_{2 \pi}^{-3 / 4}\right) \mathrm{d} t .
$$

Thus, (7.12b) follows from the fact that the function $t \rightarrow 1+|t|_{2 \pi}^{-3 / 4}$ is locally Lebesgue integrable on $\mathbb{R}$. We then obtain (7.2b) using (7.12b) and (7.11). Assertion (b) in Proposition 4.1 is proven.

\section{Proofs of Lemmas 6.1 and 2.2 (b)}

The proofs of Lemmas 6.1 and 2.2 (b) are completed in Sections 8.1 and 8.2 , respectively. Both proofs are based on properties of phase functions introduced in [5, Section 10].

8.1. Proof of Lemma 6.1. We first fix the definition of $b \frac{\omega}{n}, \underline{t}\left(j, \mathrm{e}^{\mathrm{i} \eta}\right)$ to complete that of $\mathfrak{g} \frac{\omega}{n}, \underline{t}(j)$.

8.1.1. Definition of $b \frac{\omega}{n}, \underline{t}\left(j, \mathrm{e}^{\mathrm{i} \eta}\right)$. Let $Q \frac{\omega}{n}, \underline{t}$ be the operators introduced in [5, (10.1)]. Their definition involves a phase $\tilde{\psi} \frac{\omega}{n}, \underline{t} \equiv \tilde{\psi} \frac{\omega}{n}, \underline{t}\left(j, \mathrm{e}^{\mathrm{i} \eta}\right)$ whose construction is given in [5, (10.5) and Section 10.3]. By [5, Lemma 10.3] these operators satisfy

$$
\left|H \frac{\omega}{n}, \underline{t}(j, j)-Q \frac{\omega}{n}, \underline{t}(j, j)\right| \leq \nu n^{\gamma-1+3 \varepsilon},
$$

where $\underline{\omega} \in\left(\Omega^{*}\right)^{\nu}, \underline{t} \in\left[-t_{0}, t_{0}\right]^{\nu}, n^{\varepsilon} \geq \nu$ and $n \geq \hat{n}$. Recall that $g_{\hat{n}}^{\omega}, \underline{t}(j):=H_{\frac{\omega}{\omega}}^{\underline{\omega}} \underline{\underline{t}}(j, j)$. Let similarly

$$
\tilde{\mathfrak{g}} \frac{\omega}{n}, \underline{t}(j):=Q \frac{\omega}{n}, \underline{t}(j, j) .
$$

Since $\nu n^{\gamma-1+3 \varepsilon} \leq n^{-\gamma+4 \varepsilon}$ follows from $\nu \leq n^{\varepsilon}$ and $\gamma \leq \frac{1}{2}$, the estimate (8.1) implies

$$
\left|g \frac{\omega}{n}, \underline{t}(j)-\tilde{\mathfrak{g}} \frac{\omega}{n}, \underline{t}(j)\right| \leq n^{-\gamma+4 \varepsilon} .
$$

If $|j-n| \leq n^{\gamma}$, then [5, Section 10.2.2 and (10.5)] gives the expression

$$
\tilde{\mathfrak{g}}_{n}^{\underline{\omega}, \underline{t}}(j)=\mathrm{e}^{\mathrm{i} j|\underline{\omega}|_{1}} \int_{0}^{2 \pi} \mathrm{e}^{\mathrm{i}\left(\psi \frac{\omega}{n}, \frac{t}{1}+\psi\left(\underline{\omega}, \frac{\underline{t}}{2}\right)\left(\mathrm{e}^{\mathrm{i} \eta}\right)+\mathrm{i}(j-n)\left(\varphi^{\frac{\omega}{n}, \frac{t}{1}}+\varphi_{n}, \frac{\omega}{2}, \frac{t}{2}\right)\left(\mathrm{e}^{\mathrm{i} \eta}\right)\right.} \frac{\mathrm{d} \eta}{2 \pi} .
$$

By [5, Lemma 10.1] and $\gamma \leq \frac{1}{2}$ the functions $\psi \frac{\omega}{n, t}, \varphi_{n}, \frac{\omega}{n}, \frac{t}{1}: \mathbb{T} \rightarrow \mathbb{R}$ satisfy

$$
\begin{aligned}
\left\|\psi, \frac{\omega}{n}, \frac{t}{1}\right\|_{\mathrm{C}^{2}(\mathbb{T})} & \leq C \nu n^{\gamma}, \\
\left\|\varphi \frac{\omega}{n, \frac{t}{1}}\right\|_{\mathrm{C}^{2}(\mathbb{T})} & \leq C \nu n^{\gamma-1} \leq C \nu n^{-\gamma} .
\end{aligned}
$$


By [5. Lemma 10.2] and $\gamma \leq \frac{1}{2}$ the functions $\psi \frac{\omega}{n}, \frac{t}{2}, \varphi \frac{\omega}{n}, \frac{t}{2}: \mathbb{T} \rightarrow \mathbb{R}$ satisfy

$$
\begin{aligned}
& \left\|\psi \frac{\omega}{n, \frac{t}{2}}\right\|_{\mathrm{C}^{2}(\mathbb{T})} \leq C \nu n^{\varepsilon}, \\
& \left\|\varphi_{n, \frac{\omega}{2}}\right\|_{C^{2}(\mathbb{T})} \leq C \nu n^{2(\gamma-1)+\varepsilon} \leq C n^{-2 \gamma+2 \varepsilon},
\end{aligned}
$$

for $n^{\varepsilon} \geq \nu$ and $n \geq \hat{n}$. We can rewrite (8.4) as

$$
\tilde{\mathfrak{g}} n=\underline{\underline{\omega}}, \underline{t}(j)=\mathrm{e}^{\mathrm{i} j|\underline{\omega}|_{1}} \int_{0}^{2 \pi} \mathrm{e}^{\mathrm{i} \psi \frac{\omega}{n}, \frac{t}{1}\left(\mathrm{e}^{\mathrm{i} \eta}\right)} \tilde{b} \frac{\omega}{n}, \underline{t}\left(j, \mathrm{e}^{\mathrm{i} \eta}\right) \frac{\mathrm{d} \eta}{2 \pi},
$$

with

$$
\tilde{b} \frac{\omega}{n}, \underline{t}\left(j, \mathrm{e}^{\mathrm{i} \eta}\right):=\mathrm{e}^{\mathrm{i}\left(\psi \frac{\omega}{n}, \frac{t}{2}\left(\mathrm{e}^{\mathrm{i} \eta}\right)+(j-n)\left(\varphi \frac{\omega}{n}, \frac{t}{1}+\varphi \frac{\omega}{n}, \frac{t}{2}\right)\left(\mathrm{e}^{\mathrm{i} \eta}\right)\right)} .
$$

Let us note that the definition (6.4) of $\mathfrak{g}_{n}^{\underline{\omega}, \underline{t}}(j)$ is as that of $\tilde{\mathfrak{g}} \frac{\omega}{n}, \underline{t}(j)$, replacing $\tilde{b} \frac{\omega}{n}, \underline{t}$ by $b \frac{\omega}{n}, \underline{t}$ in (8.6a).

The definition of $\psi \frac{\omega}{n}, \underline{t}$ is given in Section 6.2.1. that of $\varphi_{n, 1}^{\underline{\omega}, \underline{t}}$ and $\psi \frac{\omega}{n, \frac{t}{2}}$ are given in Sections 8.1 .3 and 8.1.6, respectively. Concerning $\varphi_{n, 2}^{\underline{\omega}, \underline{t}}$ we observe that (8.5d) ensures $(j-n) \varphi_{n, 2}^{\underline{\omega}, \underline{t}}=\mathrm{O}\left(n^{-\gamma+2 \varepsilon}\right)$ for $|j-n| \leq n^{\gamma}$, hence (8.3) still holds for these $j$ if we forget $(j-n) \varphi_{n, 2}^{\underline{\omega}, \frac{t}{2}}$ in the r.h.s. of (8.6b), i.e., if we replace $\tilde{\mathfrak{g}}_{n}^{\underline{\omega}}, \underline{t}(j)$ by $\mathfrak{g} \frac{\omega}{n}, \underline{t}(j)$ given by (6.4) with

$$
b \frac{\omega}{n}, \underline{t}\left(j, \mathrm{e}^{\mathrm{i} \eta}\right):=\mathrm{e}^{\mathrm{i}\left(\psi \frac{\omega}{n}, \frac{t}{2}\left(\mathrm{e}^{\mathrm{i} \eta}\right)+(j-n) \varphi \frac{\omega}{n}, \frac{t}{1}\left(\mathrm{e}^{\mathrm{i} \eta}\right)\right)} .
$$

Further on $b \frac{\omega}{n}, \underline{t}\left(j, \mathrm{e}^{\mathrm{i} \eta}\right)$ is given by 8.7). It is obvious that 6.13a) is satisfied and it is easy to see that the estimates (8.5b) and (8.5c) imply (6.13b). Thus, all that remains to be proved is (6.13c) and for this it suffices to prove

$$
\begin{aligned}
& \left|\partial_{t} \varphi_{n, 1}^{\underline{\omega},\left(\underline{t}^{\prime}, t\right)}\left(\mathrm{e}^{\mathrm{i} \eta}\right)\right| \leq \hat{C}_{0} n^{-\gamma}, \\
& \left|\partial_{t} \psi \frac{\omega}{n, 2}, \underline{\left.t^{\prime}, t\right)}\left(\mathrm{e}^{\mathrm{i} \eta}\right)\right| \leq \hat{C}_{0} n^{\varepsilon} .
\end{aligned}
$$

The proofs of (8.8a) and (8.8b) are given in Sections (8.1.4) and (8.1.7), respectively.

8.1.2. Change of variable $\vartheta_{n}$. As in [5, (8.3b)] we define $\varphi_{n}: \mathbb{T} \rightarrow \mathbb{R}$ by

$$
\varphi_{n}\left(\mathrm{e}^{\mathrm{i} \xi}\right):=2 \delta a(n)(1-\delta a(n) \cos \xi) \sin \xi .
$$

By $(\mathrm{H} 1 \mathrm{~b})$ we have $\delta a(n)=\mathrm{O}\left(n^{\gamma-1}\right)$. Thus we can fix $n_{0}=n_{0}\left(\left\{\varphi_{n}\right\}\right) \in \mathbb{N}$ large enough to ensure

$$
\sup _{n \geq n_{0}}\left\|\varphi_{n}\right\|_{\mathrm{C}^{2}(\mathbb{T})} \leq \frac{1}{2} \text {. }
$$

Further on we always assume $n \geq n_{0}$. Then we define the bijection $\eta_{n}: \mathbb{R} \rightarrow \mathbb{R}$ as in [5, (7.10)]:

$$
\eta_{n}(\xi):=\xi-\varphi_{n}\left(\mathrm{e}^{\mathrm{i} \xi}\right)
$$

As in [5, Section 7.5.1] we denote by $\xi_{n}: \mathbb{R} \rightarrow \mathbb{R}$ its inverse, i.e. $\xi_{n}(\eta)-\varphi_{n}\left(\mathrm{e}^{\mathrm{i} \xi_{n}(\eta)}\right)=\eta$ and, $\eta \rightarrow \xi_{n}(\eta)-\eta$ being $2 \pi$-periodic, we define $\tilde{\xi}_{n}: \mathbb{T} \rightarrow \mathbb{R}$ by $\tilde{\xi}_{n}\left(\mathrm{e}^{\mathrm{i} \eta}\right)=\xi_{n}(\eta)-\eta$. Finally, we define $\vartheta_{n}: \mathbb{T} \rightarrow \mathbb{T}$ as in $[5$, (7.13a)]:

$$
\vartheta_{n}\left(\mathrm{e}^{\mathrm{i} \eta}\right):=\mathrm{e}^{\mathrm{i} \eta} \mathrm{e}^{\mathrm{i} \tilde{\xi}_{n}\left(\mathrm{e}^{\mathrm{i} \eta}\right)}=\mathrm{e}^{\mathrm{i} \xi_{n}(\eta)} .
$$

Moreover, by [5, Lemma $9.2(\mathrm{a})]$, there exists a constant $C>0$ such that for any $q \in \mathrm{C}^{m}(\mathbb{T})$, $m=1,2,3$ one has

$$
\left\|q-q \circ \vartheta_{n}\right\|_{\mathrm{C}^{m-1}(\mathbb{T})} \leq C n^{-\gamma}\|q\|_{\mathrm{C}^{m}(\mathbb{T})}
$$


8.1.3. Definition of $\varphi_{\frac{\omega}{n}, \frac{t}{1}}$. We first assume $\nu=1$. If $(\omega, t) \in \Omega^{*} \times \mathbb{R}$ we define $\varphi_{n, 1}^{\omega, t}: \mathbb{T} \rightarrow \mathbb{R}$ for $n \geq n_{0}$ as in [5, Section 10.3.1]:

$$
\varphi_{n, 1}^{\omega, t}:=\left(\varphi_{n} \circ \tau_{\omega}-\varphi_{n}\right) \circ \vartheta_{n} \circ \tau_{t}
$$

where $\varphi_{n}, \vartheta_{n}$, and $n_{0}$ are as in Section 8.1.2 and $\tau_{t}\left(\mathrm{e}^{\mathrm{i} \xi}\right):=\mathrm{e}^{\mathrm{i}(\xi-t)}$. We have then $\varphi_{n, 1}^{\omega, t}=\varphi_{n, 1}^{\omega, 0} \circ \tau_{t}$. For $\nu \geq 2$ we define $\varphi_{n, 1}^{\underline{\omega}, t}: \mathbb{T} \rightarrow \mathbb{R}$ for $n \geq n_{0}$ by induction on $\nu$ as in [5, (10.6)]:

$$
\varphi_{n, 1}^{\underline{\omega}, \underline{t}}:=\left(\varphi_{n}^{\frac{\omega^{\prime}}{t^{\prime}}, \underline{1}}-\varphi_{n, 1}^{-\omega, t}\right) \circ \tau_{\omega},
$$

where $\underline{\omega}=\left(\underline{\omega}^{\prime}, \omega\right) \in\left(\Omega^{*}\right)^{\nu-1} \times \Omega^{*}, \underline{t}=\left(\underline{t}^{\prime}, t\right) \in \mathbb{R}^{\nu-1} \times \mathbb{R}$, and $\varphi_{n, 1}^{-\omega, t}:=\varphi_{n, 1}^{2 \pi-\omega, t}$.

8.1.4. Proof of 8.8a). By (8.10b) we get $\partial_{t} \varphi_{n, 1}^{\omega, t}=-\partial_{t} \varphi_{n, 1}^{-\omega, t} \circ \tau_{\omega}$ with $\varphi_{n, 1}^{-\omega, t}=\varphi_{n, 1}^{-\omega, 0} \circ \tau_{t}$. For any $q \in \mathrm{C}^{1}(\mathbb{T})$ we have the estimate

$$
\left|\partial_{t}\left(q \circ \tau_{t}\right)\left(\mathrm{e}^{\mathrm{i} \eta}\right)\right|=\left|q^{(1)} \circ \tau_{t}\left(\mathrm{e}^{\mathrm{i} \eta}\right)\right| \leq\|q\|_{\mathrm{C}^{1}(\mathbb{T})},
$$

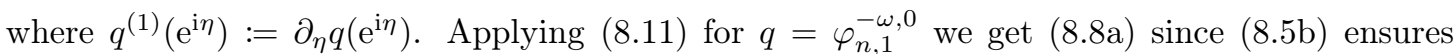
$\left\|\varphi_{n, 1}^{-\omega, 0}\right\|_{\mathrm{C}^{1}(\mathbb{T})} \leq C n^{-\gamma}$.

8.1.5. Change of variable $\vartheta_{n}^{\omega, t}$. Let $\varphi_{n, 1}^{\omega, t}$ be as above and $n_{1} \in \mathbb{N}$ such that

$$
\sup _{n \geq n_{1}}\left\|\varphi_{n, 1}^{\omega, t}\right\|_{\mathrm{C}^{2}(\mathbb{T})} \leq \frac{1}{2}
$$

It suffices to choose $n_{1}$ such that $\sup _{n \geq n_{1}}\left\|\varphi_{n, 1}\right\|_{\mathrm{C}^{2}(\mathbb{T})} \leq 1 / 4$. Indeed, using (8.9) we get

$$
\left\|\varphi_{n, 1}^{\omega, t}\right\|_{\mathrm{C}^{2}(\mathbb{T})}=\left\|\varphi_{n, 1}^{\omega, 0}\right\|_{\mathrm{C}^{2}(\mathbb{T})} \leq\left(1+C n^{-\gamma}\right)\left(\left\|\varphi_{n} \circ \tau_{\omega}\right\|_{\mathrm{C}^{3}(\mathbb{T})}+\left\|\varphi_{n}\right\|_{\mathrm{C}^{3}(\mathbb{T})}\right) \leq C^{\prime} n^{-\gamma},
$$

hence (8.12) holds if $n_{1}$ is chosen so that $C n_{1}^{-\gamma} \leq 1 / 2$. From now on we assume $n \geq n_{1}$ and introduce the bijection $\eta_{n}^{\omega, t}: \mathbb{R} \rightarrow \mathbb{R}$ by

$$
\eta_{n}^{\omega, t}(\xi):=\xi-\varphi_{n, 1}^{\omega, t}\left(\mathrm{e}^{\mathrm{i} \xi}\right) .
$$

Let $\xi_{n}^{\omega, t}: \mathbb{R} \rightarrow \mathbb{R}$ be its inverse. Then $\xi_{n}^{\omega, t}(\eta)-\varphi_{n, 1}^{\omega, t}\left(\mathrm{e}^{\mathrm{i} \xi_{n}^{\omega, t}(\eta)}\right)=\eta$ and $\eta \rightarrow \xi_{n}^{\omega, t}(\eta)-\eta$ is $2 \pi$-periodic. As before we can define $\tilde{\xi}_{n}^{\omega, t}: \mathbb{T} \rightarrow \mathbb{R}$ by the formula $\tilde{\xi}_{n}^{\omega, t}\left(\mathrm{e}^{\mathrm{i} \eta}\right)=\xi_{n}^{\omega, t}(\eta)-\eta$ and $\vartheta_{n}^{\omega, t}: \mathbb{T} \rightarrow \mathbb{T}$ by

$$
\vartheta_{n}^{\omega, t}\left(\mathrm{e}^{\mathrm{i} \eta}\right):=\mathrm{e}^{\mathrm{i} \eta} \mathrm{e}^{\mathrm{i} \tilde{\xi}_{n}^{\omega, t}\left(\mathrm{e}^{\mathrm{i} \eta}\right)}=\mathrm{e}^{\mathrm{i} \xi_{n}^{\omega, t}(\eta)} .
$$

Since we can use $\varphi_{n, 1}^{\omega, 0}$ in place of $\varphi_{n}$ in [5], proof of Lemma 9.2], there is a constant $C>0$ such that for any $q \in \mathrm{C}^{2}(\mathbb{T})$ one has

$$
\left\|q-q \circ \vartheta_{n}^{\omega, 0}\right\|_{\mathrm{C}^{1}(\mathbb{T})} \leq C n^{-\gamma}\|q\|_{\mathrm{C}^{2}(\mathbb{T})} .
$$

Lemma 8.1. (a) One has

$$
\vartheta_{n}^{\omega, t}=\tau_{-t} \circ \vartheta_{n}^{\omega, 0} \circ \tau_{t} .
$$

(b) There is a constant $C>0$ such that

$$
\left|\partial_{t} \vartheta_{n}^{\omega, t}\left(\mathrm{e}^{\mathrm{i} \xi}\right)\right| \leq C n^{-\gamma} .
$$

Proof. (a) We first note that (8.10a) implies $\varphi_{n, 1}^{\omega, t}=\varphi_{n, 1}^{\omega, 0} \circ \tau_{t}$. Using this relation in (8.13) we find $\eta_{n}^{\omega, t}(\xi)=t+\eta_{n}^{\omega, 0}(\xi-t)$, hence also $\xi_{n}^{\omega, t}(\eta)=t+\xi_{n}^{\omega, 0}(\eta-t)$. We have then $\tilde{\xi}_{n}^{\omega, t}\left(\mathrm{e}^{\mathrm{i} \eta}\right):=$ $\xi_{n}^{\omega, t}(\eta)-\eta=\xi_{n}^{\omega, 0}(\eta-t)-(\eta-t)=\tilde{\xi}_{n}^{\omega, 0}\left(\mathrm{e}^{\mathrm{i}(\eta-t)}\right)$, i.e.,

$$
\tilde{\xi}_{n}^{\omega, t}=\tilde{\xi}_{n}^{\omega, 0} \circ \tau_{t},
$$

and (8.16) follows from (8.18) and (8.14). 
(b) Since $\tilde{\xi}_{n}^{\omega, t}=\tilde{\xi}_{n}^{\omega, 0} \circ \tau_{t}$ the estimate (8.11) ensures

$$
\left|\partial_{t} \vartheta_{n}^{\omega, t}\left(\mathrm{e}^{\mathrm{i} \eta}\right)\right|=\left|\partial_{t} \tilde{\xi}_{n}^{\omega, t}\left(\mathrm{e}^{\mathrm{i} \eta}\right)\right| \leq\left\|\tilde{\xi}_{n}^{\omega, 0}\right\|_{\mathrm{C}^{1}(\mathbb{T})}
$$

and the right hand side of (8.19) is $\mathrm{O}\left(n^{-\gamma}\right)$ due to [5, Lemma 7.3] with $\varphi_{n, 1}^{\omega, 0}$ in place of $\varphi_{n}$.

8.1.6. Definition of $\psi \frac{\omega}{n}, \frac{t}{2}$. For $\omega \in \Omega^{*}$ we denote

$$
\begin{aligned}
\psi_{n, \mathrm{I}}^{\omega} & :=\psi_{n, 2} \circ \tau_{\omega}-\psi_{n, 2}, \\
\psi_{n, \mathrm{II}}^{\omega} & :=\psi_{n, 1}^{\omega, 0} \circ \vartheta_{n}-\psi_{n, 1}^{\omega, 0},
\end{aligned}
$$

where $\psi_{n, 1}^{\omega, 0}\left(\mathrm{e}^{\mathrm{i} \xi}\right)=-4 a(n) \sin \frac{\omega}{2} \cos \left(\xi-\frac{\omega}{2}\right)$ as in (6.7) and

$$
\psi_{n, 2}\left(\mathrm{e}^{\mathrm{i} \xi}\right):=-a(n) \delta a(n) \sin 2 \xi,
$$

as in [5, $(9.7 \mathrm{c})]$. A direct computation gives the expression

$$
\psi_{n, \mathrm{I}}^{\omega}\left(\mathrm{e}^{\mathrm{i} \xi}\right)=2 a(n) \delta a(n) \sin \omega \cos (2 \xi-\omega) .
$$

For $\nu=1,(\omega, t) \in \Omega^{*} \times \mathbb{R}$ we define $\psi_{n, 2}^{\omega, t}$ as in [5, Section 9.3.2], i.e.,

$$
\psi_{n, 2}^{\omega, t}:=\left(\psi_{n, \mathrm{II}}^{\omega}+\psi_{n, \mathrm{I}}^{\omega} \circ \vartheta_{n}\right) \circ \tau_{t} .
$$

For $\nu \geq 2, \underline{\omega}=\left(\underline{\omega}^{\prime}, \omega\right) \in\left(\Omega^{*}\right)^{\nu-1} \times \Omega^{*}, \underline{t}=\left(\underline{t}^{\prime}, t\right) \in \mathbb{R}^{\nu-1} \times \mathbb{R}$, we use the same induction formula as in [5, (10.12)], i.e.,

$$
\psi \frac{\omega}{n, \frac{t}{2}}=\left(\psi_{n, 1}^{-\omega, t}-\psi_{n, 1}^{-\omega, t} \circ \vartheta_{n}^{\omega, t}+\psi \frac{\omega^{\prime}, \underline{t}^{\prime}}{n, 2}-\psi_{n, 2}^{-\omega, t}+\psi \frac{\omega^{\prime}, \underline{t}^{\prime}}{1^{\prime}} \circ \vartheta_{n}^{\omega, t}-\psi \frac{\omega^{\prime}, \underline{t}^{\prime}}{1}\right) \circ \tau_{\omega},
$$

where $\psi_{n, i}^{-\omega, t}:=\psi_{n, i}^{2 \pi-\omega, t}, i=1,2$.

8.1.7. End of the proof of Lemma 6.1. We recall that 8.8a was proved in Section 8.1.4 and following the remark from Section 8.1.1 it only remains to prove (8.8b) , i.e. $\left|\partial_{t} \psi \frac{\omega}{n}, \frac{t}{2}\left(\mathrm{e}^{\mathrm{i} \eta}\right)\right| \leq \hat{C}_{0} n^{\varepsilon}$ where $t=t_{\nu}$.

If $\nu=1$ then $\psi_{n, 2}^{\omega, t}=\psi_{n, 2}^{\omega, 0} \circ \tau_{t}$ and (8.8b follows from (8.5c) using (8.11).

If $\nu \geq 2$ then $\psi \frac{\omega}{n, 2}, \frac{t}{2}$ is given by (8.24b). To estimate $\partial_{t} \psi \frac{\omega}{n, \frac{t}{2}}$ we first observe that

$$
\psi_{n, 1}^{-\omega, t}-\psi_{n, 1}^{-\omega, t} \circ \vartheta_{n}^{\omega, t}=\left(\psi_{n, 1}^{-\omega, 0}-\psi_{n, 1}^{-\omega, 0} \circ \vartheta_{n}^{\omega, 0}\right) \circ \tau_{t}
$$

and (8.11) allows us to estimate

$$
\left|\partial_{t}\left(\psi_{n, 1}^{-\omega, t}-\psi_{n, 1}^{-\omega, t} \circ \vartheta_{n}^{\omega, t}\right)\left(\mathrm{e}^{\mathrm{i} \eta}\right)\right| \leq\left\|\psi_{n, 1}^{-\omega, 0} \circ \vartheta_{n}^{\omega, 0}-\psi_{n, 1}^{-\omega, 0}\right\|_{\mathrm{C}^{1}(\mathbb{T})} .
$$

The right hand side of (8.25) can be estimated by a constant $C$ independent of $n$ by using 8.15) for $q=\psi_{n, 1}^{-\omega, 0}$ and (8.5a), i.e., $\left\|\psi_{n, 1}^{-\omega, 0}\right\|_{\mathrm{C}^{1}(\mathbb{T})}=\mathrm{O}\left(n^{\gamma}\right)$. It remains to estimate

$$
\left|\partial_{t}\left(\psi_{n, 1}^{\omega^{\prime}, \underline{t}^{\prime}} \circ \vartheta_{n}^{\omega, t}\right)\left(\mathrm{e}^{\mathrm{i} \eta}\right)\right| \leq\left\|\psi{\frac{\omega}{n}, \underline{\omega}^{\prime}}^{\prime}\right\|_{\mathrm{C}^{1}(\mathbb{T})}\left|\partial_{t} \vartheta_{n}^{\omega, t}\left(\mathrm{e}^{\mathrm{i} \eta}\right)\right| .
$$

However (8.17) and (8.5a) allow us to estimate the right hand side of (8.26) by $C \nu \leq C n^{\varepsilon}$ which completes the proof of (8.8b). 
8.2. Proof of Lemma 2.2 (b). This section is devoted to the proof of Lemma 2.2 under assumptions (H1) and (H2). Let $\psi_{n, \mathrm{I}}^{\omega}, \psi_{n, \mathrm{II}}^{\omega}$ be given by (8.20) and (8.21), respectively. Then

$$
\psi_{n, 2}^{\omega, 0}=\psi_{n, \mathrm{I}}^{\omega}+\psi_{n, \mathrm{II}}^{\omega}+r_{n}^{\omega}
$$

holds with

$$
r_{n}^{\omega}:=\psi_{n, \mathrm{I}}^{\omega} \circ \vartheta_{n}-\psi_{n, \mathrm{I}}^{\omega} .
$$

Lemma 8.2. We have the estimates

$$
\begin{aligned}
& \left\|r_{n}^{\omega}\right\|_{\mathrm{C}^{0}(\mathbb{T})}=\mathrm{O}\left(n^{-\gamma}\right), \\
& \left|\psi_{n, \mathrm{II}}^{\omega}\left( \pm \mathrm{e}^{\mathrm{i} \omega / 2}\right)\right|=\mathrm{O}\left(n^{-\gamma}\right) .
\end{aligned}
$$

Proof. We observe that (8.9) ensures

$$
\left\|\psi_{n, \mathrm{I}}^{\omega} \circ \vartheta_{n}-\psi_{n, \mathrm{I}}^{\omega}\right\|_{\mathrm{C}^{0}(\mathbb{T})} \leq C n^{-\gamma}\left\|\psi_{n, \mathrm{I}}^{\omega}\right\|_{\mathrm{C}^{1}(\mathbb{T})} .
$$

To complete the proof of (8.29) it suffices to observe that $\left\|\psi_{n, \mathrm{I}}^{\omega}\right\|_{\mathrm{C}^{1}(\mathbb{T})}=\mathrm{O}(1)$.

To prove (8.30) we write the Taylor expansion at order 2:

$$
\psi_{n, \mathrm{II}}^{\omega}\left(\mathrm{e}^{\mathrm{i} \omega / 2}\right)=\partial_{\eta} \psi_{n, 1}^{\omega, 0}\left(\mathrm{e}^{\mathrm{i} \omega / 2}\right)\left(\vartheta_{n}\left(\mathrm{e}^{\mathrm{i} \omega / 2}\right)-\mathrm{e}^{\mathrm{i} \omega / 2}\right)+\tilde{r}_{n}^{\omega}
$$

with

$$
\left|\tilde{r}_{n}^{\omega}\right| \leq\left\|\psi_{n, 1}^{\omega, 0}\right\|_{\mathrm{C}^{2}(\mathbb{T})}\left|\vartheta_{n}\left(\mathrm{e}^{\mathrm{i} \omega / 2}\right)-\mathrm{e}^{\mathrm{i} \omega / 2}\right|^{2} \leq C n^{\gamma}\left|\tilde{\xi}_{n}\left(\mathrm{e}^{\mathrm{i} \omega / 2}\right)\right|^{2} \leq C^{\prime} n^{-\gamma}
$$

A similar reasoning can be applied to $-\mathrm{e}^{\mathrm{i} \omega / 2}$ instead of $\mathrm{e}^{\mathrm{i} \omega / 2}$ and to complete the proof of (8.30) we observe that $\partial_{\eta} \psi_{n, 1}\left( \pm \mathrm{e}^{\mathrm{i} \omega / 2}\right)=0$.

To begin the proof of (2.30) in case (b) we observe that

$$
g_{n}(n)=\sum_{m=1}^{\lfloor N / 2\rfloor} \alpha_{m} \operatorname{Re}\left\langle\mathrm{e}_{n}, H_{n}^{2 \pi m / N, 0} \mathrm{e}_{n}\right\rangle+\sum_{m=1}^{\lfloor(N-1) / 2\rfloor} \tilde{\alpha}_{m} \operatorname{Im}\left\langle\mathrm{e}_{n}, H_{n}^{2 \pi m / N, 0} \mathrm{e}_{n}\right\rangle
$$

and Lemma 6.1 in the case $\nu=1$ ensures

$$
g_{n}(n)=\sum_{m=1}^{\lfloor N / 2\rfloor} \alpha_{m} \operatorname{Re} \mathfrak{g}_{n}^{2 \pi m / N, 0}(n)+\sum_{m=1}^{\lfloor(N-1) / 2\rfloor} \operatorname{Im} \mathfrak{g}_{n}^{2 \pi m / N, 0}(n)+\mathrm{O}\left(n^{-\gamma+5 \varepsilon}\right)
$$

with

for $\omega \in \Omega^{*}$ and

$$
\mathfrak{g}_{n}^{\omega, 0}(n)=\mathrm{e}^{\mathrm{i} \omega n} \int_{0}^{2 \pi} \mathrm{e}^{\mathrm{i} \psi_{n, 1}^{\omega, 0}\left(\mathrm{e}^{\mathrm{i} \eta}\right)} b_{n}^{\omega}\left(\mathrm{e}^{\mathrm{i} \eta}\right) \frac{\mathrm{d} \eta}{2 \pi}
$$

$$
b_{n}^{\omega}\left(\mathrm{e}^{\mathrm{i} \eta}\right)=\mathrm{e}^{\mathrm{i} \psi_{n, 2}^{\omega, 0}\left(\mathrm{e}^{\mathrm{i} \eta}\right)}
$$

according to (8.7). Then Lemma 3.1 with $\mu=4 a(n) \sin \frac{\omega}{2}$ and $\eta_{0}=\pi+\frac{\omega}{2}$ gives

$$
\mathfrak{g}_{n}^{\omega, 0}(n)=\mathrm{e}^{\mathrm{i} \omega n} \sum_{\kappa= \pm 1} \frac{\mathrm{e}^{\mathrm{i} \kappa\left(4 a(n) \sin \frac{\omega}{2}-\frac{\pi}{4}\right)}}{2 \sqrt{2 \pi a(n) \sin \frac{\omega}{2}}} b_{n}^{\omega}\left(-\kappa \mathrm{e}^{\mathrm{i} \omega / 2}\right)+\mathrm{O}\left(a(n)^{-1}\right)
$$

and applying Lemma 8.2 we obtain

$$
b_{n}^{\omega}\left( \pm \mathrm{e}^{\mathrm{i} \omega / 2}\right)=\mathrm{e}^{\mathrm{i} \psi_{n, \mathrm{I}}^{\omega}\left( \pm \mathrm{e}^{\mathrm{i} \omega / 2}\right)}+\mathrm{O}\left(n^{-\gamma}\right) .
$$

However using (8.23) we get $\psi_{n, \mathrm{I}}^{\omega}\left( \pm \mathrm{e}^{\mathrm{i} \omega / 2}\right)=2 a(n) \delta a(n) \sin \omega$. Thus combining (8.32) with (8.31) and $a(n)^{-1}=\mathrm{O}\left(n^{-\gamma}\right)$, we obtain

$$
\mathfrak{g}_{n}^{\omega, 0}(n)=\frac{\cos \left(4 a(n) \sin \frac{\omega}{2}-\frac{\pi}{4}\right)}{\sqrt{2 \pi a(n) \sin \frac{\omega}{2}}} \mathrm{e}^{\mathrm{i}(\omega n+2 a(n) \delta a(n) \sin \omega)}+\mathrm{O}\left(n^{-\gamma}\right) .
$$


To complete the proof we observe that (8.33) ensures

$$
\begin{aligned}
& \operatorname{Re} \mathfrak{g}_{n}^{\omega, 0}(n)=\frac{\cos \left(4 a(n) \sin \frac{\omega}{2}-\frac{\pi}{4}\right)}{\sqrt{2 \pi a(n) \sin \frac{\omega}{2}}} \cos (\omega n+2 a(n) \delta a(n) \sin \omega)+\mathrm{O}\left(n^{-\gamma}\right), \\
& \operatorname{Im} \mathfrak{g}_{n}^{\omega, 0}(n)=\frac{\cos \left(4 a(n) \sin \frac{\omega}{2}-\frac{\pi}{4}\right)}{\sqrt{2 \pi a(n) \sin \frac{\omega}{2}}} \sin (\omega n+2 a(n) \delta a(n) \sin \omega)+\mathrm{O}\left(n^{-\gamma}\right) .
\end{aligned}
$$

\section{Appendix A. The QuAntum Rabi MOdel}

The quantum Rabi model couples a quantized single-mode radiation and a two-level quantum system.

Let $\mathcal{H}_{\text {field }}$ be a complex Hilbert space equipped with an orthonormal basis $\left\{\mathrm{e}_{n}\right\}_{0}^{\infty}$ and let $\hat{a}$, $\hat{a}^{\dagger}$ be the photon annihilation and creation operators defined in $\mathcal{H}_{\text {field }}$ by

$$
\begin{aligned}
\hat{a} \mathrm{e}_{n} & =\sqrt{n} \mathrm{e}_{n-1}, & & n=0,1,2, \ldots, \\
\hat{a}^{\dagger} \mathrm{e}_{n} & =\sqrt{n+1} \mathrm{e}_{n+1}, & & n=0,1,2, \ldots
\end{aligned}
$$

(with $\mathrm{e}_{-1}=0$ ). To define the quantum Rabi model we fix four positive parameters:

(i) $\omega$, the frequency of the quantized one-mode electromagnetic field,

(ii) $E$, the level separation energy,

(iii) $g$, the coupling constant,

(iv) $\hbar$, the Planck constant.

The quantum Rabi Hamiltonian is then the self-adjoint operator in $\mathcal{H}_{\text {field }} \otimes \mathbb{C}^{2}$ given by

$$
H_{\mathrm{Rabi}}=\hbar \omega \hat{a}^{\dagger} \hat{a} \otimes I_{\mathbb{C}^{2}}+I_{\mathcal{H}_{\text {field }}} \otimes \frac{1}{2} E \sigma_{z}+\hbar g\left(\hat{a}^{\dagger}+\hat{a}\right) \otimes \sigma_{x},
$$

where $\sigma_{x}=\left(\begin{array}{ll}0 & 1 \\ 1 & 0\end{array}\right)$ and $\sigma_{z}=\left(\begin{array}{cc}1 & 0 \\ 0 & -1\end{array}\right)$. Then we have the decomposition (see [19] or [6, Section 3.1])

$$
\mathcal{H}_{\text {field }} \otimes \mathbb{C}^{2}=\mathcal{H}_{+} \oplus \mathcal{H}_{-},
$$

where $\mathcal{H}_{+}$and $\mathcal{H}_{-}$are invariant under $H_{\text {Rabi }}$ and the restrictions $H_{ \pm}:=\left.H_{\text {Rabi }}\right|_{\mathcal{H}_{ \pm}}$have the form

$$
H_{ \pm}=-\frac{1}{2} \hbar \omega+\hbar \omega J_{ \pm}
$$

where the operator $J_{+}$(resp. $J_{-}$) is defined in an appropriate basis by the Jacobi matrix (1.1) whose entries are given by (1.2) with $a_{1}=\frac{g}{\omega}$ and $\rho=\frac{E}{2 \hbar \omega}$ (resp. $\rho=-\frac{E}{2 \hbar \omega}$ ). Therefore

$$
\sigma\left(H_{\text {Rabi }}\right)=\left\{\lambda_{n}\left(H_{+}\right)\right\}_{n=1}^{\infty} \cup\left\{\lambda_{n}\left(H_{-}\right)\right\}_{n=1}^{\infty},
$$

where

$$
\lambda_{n}\left(H_{ \pm}\right)=-\frac{1}{2} \hbar \omega+\hbar \omega \lambda_{n}\left(J_{ \pm}\right)
$$

\section{REFERENCES}

[1] A. Boutet de Monvel, S. Naboko, and L. O. Silva, The asymptotic behavior of eigenvalues of a modified Jaynes-Cummings model, Asymptot. Anal. 47 (2006), no. 3-4, 291-315.

[2] A. Boutet de Monvel and L. Zielinski, Eigenvalue asymptotics for Jaynes-Cummings type models without modulations, BiBoS preprint 08-03-278 (2008), available at http://www.physik.uni-bielefeld.de/bibos/

[3] _ Explicit error estimates for eigenvalues of some unbounded Jacobi matrices, Spectral Theory, Mathematical System Theory, Evolution Equations, Differential and Difference Equations: IWOTA10, Oper. Theory Adv. Appl., vol. 221, Birkhäuser Verlag, Basel, 2012, pp. 187-215.

[4] _ - Asymptotic behavior of large eigenvalues of a modified Jaynes-Cummings model, Spectral Theory and Differential Equations, Amer. Math. Soc. Transl. Ser. 2, vol. 233, Amer. Math. Soc., Providence, RI, 2014, pp. 77-93.

[5] _ Asymptotic behavior of large eigenvalues for Jaynes-Cummings type models, J. Spectr. Theory 7 (2017), no. 2, 559-631.

[6] - On the spectrum of the quantum Rabi model, submitted. 
[7] D. Braak, Integrability of the Rabi Model, Phys. Rev. Lett. 107 (2011), no. 10, 100401, 4 pp.

[8] D. Braak, Q.-H. Chen, M. T. Batchelor, and E. Solano, Semi-classical and quantum Rabi models: in celebration of 80 years, J. Phys. A: Math. Theor. 49 (2016), no. 30, 300301, 4 pp.

[9] I. D. Feranchuk, L. I. Komarov, and A. P. Ulyanenkov, Two-level system in a one-mode quantum field: numerical solution on the basis of the operator method, J. Phys. A: Math. Gen. 29 (1996), no. 14, 4035-4047.

[10] I. D. Feranchuk, A. Ivanov, V.-H. Le, and A. P. Ulyanenkov, Non-perturbative Description of Quantum Systems, Lecture Notes in Physics, vol. 894, Springer, Cham, 2015.

[11] S. He, Y.-Y. Zhang, Q.-H. Chen, X.-Z. Ren, T. Liu, and K.-L. Wang, Unified analytical treatments of qubitoscillator systems, Chinese Phys. B 22 (2013), no. 6, 064205.

[12] E. K. Irish, Generalized Rotating-Wave Approximation for Arbitrarily Large Coupling, Phys. Rev. Lett. 99 (2007), no. 17,173601

[13] J. Janas and S. Naboko, Infinite Jacobi matrices with unbounded entries: asymptotics of eigenvalues and the transformation operator approach, SIAM J. Math. Anal. 36 (2004), no. 2, 643-658.

[14] E. T. Jaynes and F. W. Cummings, Comparison of quantum and semiclassical radiation theories with application to the beam maser, Proc. IEEE 51 (1963), no. 1, 89-109.

[15] M. Malejki, Asymptotics of large eigenvalues for some discrete unbounded Jacobi matrices, Linear Algebra Appl. 431 (2009), no. 10, 1952-1970.

[16] M. Schmutz, Two-level system coupled to a boson mode: the large n limit, J. Phys. A: Math. Gen. 19 (1986), no. $17,3565-3577$.

[17] M. O. Scully and M. S. Zubairy, Quantum Optics, Cambridge University Press, Cambridge, 1997.

[18] E. M. Stein, Harmonic analysis: real-variable methods, orthogonality, and oscillatory integrals, Princeton Mathematical Series, vol. 43, Princeton University Press, Princeton, NJ, 1993. With the assistance of Timothy S. Murphy; Monographs in Harmonic Analysis, III.

[19] È. A. Tur, Jaynes-Cummings model: solution without rotating wave approximation, Optics and Spectroscopy 89 (2000), no. 4, 574-588.

[20] E. A. Tur, Jaynes-Cummings model without rotating wave approximation. Asymptotics of eigenvalues (2002), 12 pp., available at arXiv.org/abs/math-ph/0211055

[21] Q. Xie, H. Zhong, M. T. Batchelor, and C. Lee, The quantum Rabi model: solution and dynamics, J. Phys. A: Math. Theor. 50 (2017), no. 11, 113001, 40 pp.

[22] E. A. Yanovich, Asymptotics of eigenvalues of an energy operator in a problem of quantum physics, Operator Methods in Mathematical Physics, Oper. Theory Adv. Appl., vol. 227, Birkhäuser/Springer Basel AG, Basel, 2013, pp. 165-177.

${ }^{1}$ Institut de Mathématiques de Jussieu-PRG, Université Paris Diderot, bâtiment Sophie Germain, case 7012, 75205 Paris Cedex 13, France, E-mail: anne.boutet-de-monvel@imu-Prg.fr

${ }^{2}$ Université du Littoral Côte d'Opale, Laboratoire de Mathématiques Pures et Appliquées, 62100 Calais, France, E-mail: Lech.Zielinski@lmpa.univ-littoral.Fr 